\title{
Diabetes Mellitus and Cardiovascular Risk Assessment in Mothers with a History of Gestational Diabetes Mellitus Based on Postpartal Expression Profile of MicroRNAs Associated with Diabetes Mellitus and Cardiovascular and Cerebrovascular Diseases
}

\author{
Ilona Hromadnikova ${ }^{1, *(\mathbb{D}}$, Katerina Kotlabova ${ }^{1}$, Lenka Dvorakova ${ }^{1}$ and Ladislav Krofta ${ }^{2}$ \\ 1 Department of Molecular Biology and Cell Pathology, Third Faculty of Medicine, Charles University, \\ 10000 Prague, Czech Republic; katerina.kotlabova@lf3.cuni.cz (K.K.); lenka.dvorakova@lf3.cuni.cz (L.D.) \\ 2 Institute for the Care of the Mother and Child, Third Faculty of Medicine, Charles University, 14700 Prague, \\ Czech Republic; ladislav.krofta@upmd.eu \\ * Correspondence: ilona.hromadnikova@lf3.cuni.cz; Tel.: +420-296-511-336
}

Received: 11 February 2020; Accepted: 30 March 2020; Published: 31 March 2020

check for updates

\begin{abstract}
Mothers with a history of gestational diabetes mellitus (GDM) have an increased risk of developing diabetes in the future and a lifelong cardiovascular risk. Postpartal expression profile of cardiovascular/cerebrovascular disease associated microRNAs was assessed 3-11 years after the delivery in whole peripheral blood of young and middle-aged mothers with a prior exposure to GDM with the aim to identify a high-risk group of mothers at risk of later development of diabetes mellitus and cardiovascular/cerebrovascular diseases who would benefit from implementation of early primary prevention strategies and long-term follow-up. The hypothesis of the assessment of cardiovascular risk in women was based on the knowledge that a series of microRNAs play a role in the pathogenesis of diabetes mellitus and cardiovascular/cerebrovascular diseases. Abnormal expression profile of multiple microRNAs was found in women with a prior exposure to GDM (miR-1-3p, miR-16-5p, miR-17-5p, miR-20a-5p, miR-20b-5p, miR-21-5p, miR-23a-3p, miR-24-3p, miR-26a-5p, miR-29a-3p, miR-100-5p, miR-103a-3p, miR-125b-5p, miR-126-3p, miR-130b-3p, miR-133a-3p, miR-143-3p, miR-145-5p, miR-146a-5p, miR-181a-5p, miR-195-5p, miR-199a-5p, miR-221-3p, miR-342-3p, miR-499a-5p, and-miR-574-3p). Postpartal combined screening of miR-1-3p, miR-16-5p, miR-17-5p, miR-20b-5p, miR-21-5p, miR-23a-3p, miR-26a-5p, miR-29a-3p, miR-103a-3p, miR-133a-3p, miR-146a-5p, miR-181a-5p, miR-195-5p, miR-199a-5p, miR-221-3p, and miR-499a-5p showed the highest accuracy for the identification of mothers with a prior exposure to GDM at a higher risk of later development of cardiovascular/cerebrovascular diseases (AUC 0.900, $p<0.001$, sensitivity $77.48 \%$, specificity $93.26 \%$, cut off $>0.611270413$ ). It was able to identify $77.48 \%$ mothers with an increased cardiovascular risk at $10.0 \%$ FPR. Any of changes in epigenome (upregulation of miR-16-5p, miR-17-5p, miR-29a-3p, and miR-195-5p) that were induced by GDM-complicated pregnancy are long-acting and may predispose mothers affected with GDM to later development of diabetes mellitus and cardiovascular/cerebrovascular diseases. In addition, novel epigenetic changes (upregulation of serious of microRNAs) appeared in a proportion of women that were exposed to GDM throughout the postpartal life. Likewise, a previous occurrence of either GH, PE, and/or FGR, as well as a previous occurrence of GDM, is associated with the upregulation of miR-1-3p, miR-17-5p, miR-20a-5p, miR-20b-5p, miR-29a-3p, miR-100-5p, miR-125b-5p, miR-126-3p, miR-130b-3p, miR-133a-3p, miR-143-3p, miR-145-5p, miR-146a-5p, miR-181a-5p, miR-199a-5p, miR-221-3p, and miR-499a-5p. On the other hand, upregulation of miR-16-5p, miR-21-5p, miR-23a-3p, miR-24-3p, miR-26a-5p, miR-103a-3p, miR-195-5p, miR-342-3p, and miR-574-3p represents a unique feature
\end{abstract}


of aberrant expression profile of women with a prior exposure to GDM. Screening of particular microRNAs may stratify a high-risk group of mothers with a history of GDM who might benefit from implementation of early primary prevention strategies.

Keywords: cardiovascular/cerebrovascular diseases; cardiovascular risk; gestational diabetes mellitus; microRNA expression; mothers; pregnancy complications; primary prevention; screening

\section{Introduction}

Recently, we demonstrated that history of pregnancy-related complications, such as gestational hypertension (GH), preeclampsia (PE), and/or fetal growth restriction (FGR), was associated with postpartum epigenetic changes characteristic for cardiovascular and cerebrovascular diseases [1-3].

We showed that previous occurrence of either GH, PE, and/or FGR might predispose a proportion of women to later development of cardiovascular and cerebrovascular diseases due to the presence of alterations in the expression of cardiovascular and cerebrovascular disease-associated microRNAs in their whole peripheral blood [1-3].

Gestational diabetes mellitus (GDM) is defined as carbohydrate intolerance that develops during pregnancy, usually during second or third trimester of gestation [4]. Women with GDM have an increased risk of developing diabetes (predominantly type 2 diabetes) later in life. It is estimated that up to $70 \%$ of women with GDM will develop diabetes within 22-28 years after pregnancy [4-7].

The incidence of GDM increases with the same risk factors seen for type 2 diabetes such as obesity, sedentary lifestyle, and increasing reproductive age of women [4-7].

The initial criteria for diagnosis of GDM were established more than 40 years ago to identify women at a high risk for development of diabetes after pregnancy [8-10]. The two-step screening approach to testing for GDM is currently recommended by The American College of Obstetricians and Gynecologists [4] and The International Association of Diabetes and Pregnancy Study Groups (IADPSG) [10]. Overall, using the proposed IADPSG criteria, 18\% of pregnant women in the United States are identified as having GDM [10].

The goal of the current study was to evaluate the risk of later development of diabetes mellitus, and cardiovascular and cerebrovascular diseases based on an epigenetic profile of cardiovascular/cerebrovascular disease associated microRNAs in whole peripheral blood of young and middle-aged mothers with a history of gestational diabetes mellitus (GDM) 3-11 years after the delivery. The hypothesis of the assessment of cardiovascular risk in women was based on the knowledge that a series of microRNAs play a role in the pathogenesis of diabetes mellitus and cardiovascular/cerebrovascular diseases (Table 1) [11-170].

Postpartal epigenetic profiling of microRNAs (miR-1-3p, miR-16-5p, miR-17-5p, miR-20a-5p, miR-20b-5p, miR-21-5p, miR-23a-3p, miR-24-3p, miR-26a-5p, miR-29a-3p, miR-92a-3p, miR-100-5p, miR-103a-3p, miR-125b-5p, miR-126-3p， miR-130b-3p， miR-133a-3p， miR-143-3p， miR-145-5p， miR-146a-5p, miR-155-5p, miR-181a-5p, miR-195-5p, miR-199a-5p, miR-210-3p, miR-221-3p, miR-342-3p, miR-499a-5p, and miR-574-3p) known to be involved in the onset of insulin resistance and diabetes, dyslipidemia, hypertension, vascular inflammation, atherosclerosis, angiogenesis, coronary artery disease, myocardial infarction and heart failure, stroke, intracranial aneurysm, pulmonary arterial hypertension, and peripartum cardiomyopathy was the subject of our interest.

MicroRNAs, small noncoding RNAs, are known to regulate gene expression at the posttranscriptional level by blocking translation or degrading of target messenger RNA [171].

To the best of our present knowledge, no study on expression profiling of microRNAs associated with diabetes mellitus, and cardiovascular and cerebrovascular diseases in whole peripheral blood of mothers after pregnancies affected by gestational diabetes mellitus has been carried out. 
Table 1. The role of differentially expressed microRNAs in mothers with a history of GDM in the pathogenesis of diabetes mellitus, cardiovascular/cerebrovascular diseases.

\begin{tabular}{|c|c|c|}
\hline miRBase ID & $\begin{array}{l}\text { Gene Location on } \\
\text { Chromosome }\end{array}$ & Role in the Pathogenesis of Cardiovascular/Cerebrovascular Diseases \\
\hline hsa-miR-1-3p & $\begin{array}{c}20 \mathrm{q} 13.3 \\
18 \mathrm{q} 11.2[11]\end{array}$ & $\begin{array}{c}\text { Acute myocardial infarction, heart ischemia, post-myocardial infarction complications [12], diabetes mellitus [13,14], } \\
\text { vascular endothelial dysfunction [15] }\end{array}$ \\
\hline hsa-miR-16-5p & $13 q 14.2$ & $\begin{array}{l}\text { Myocardial infarction [16,17], heart failure [18], acute coronary syndrome, cerebral ischaemic events [19], gestational } \\
\text { diabetes mellitus [20,21], diabetes mellitus [22-24] }\end{array}$ \\
\hline hsa-miR-17-5p & $13 q 31.3[25,26]$ & $\begin{array}{l}\text { Cardiac development [27], ischemia/reperfusion-induced cardiac injury [28], kidney ischemia-reperfusion injury [29], } \\
\text { diffuse myocardial fibrosis in hypertrophic cardiomyopathy [30], acute ischemic stroke [31], coronary artery disease [32], } \\
\text { adipogenic differentiation [33], gestational diabetes mellitus [20,21], diabetes mellitus [24,34] }\end{array}$ \\
\hline hsa-miR-20a-5p & $13 q 31.3[35]$ & $\begin{array}{l}\text { Pulmonary hypertension [36], gestational diabetes mellitus [20,21,37], diabetic retinopathy [38], diabetes with abdominal } \\
\text { aortic aneurysm [39] }\end{array}$ \\
\hline hsa-miR-20b-5p & Xq26.2 [35] & Hypertension-induced heart failure [40], insulin resistance [41], T2DM [42,43], diabetic retinopathy [44] \\
\hline hsa-miR-21-5p & $17 q 23.2[45]$ & $\begin{array}{l}\text { Homeostasis of the cardiovascular system [46], cardiac fibrosis and heart failure [47,48], ascending aortic aneurysm [49], } \\
\text { regulation of hypertension-related genes [50], myocardial infarction [51], insulin resistance [41], T2DM [52], T2DM with } \\
\text { major cardiovascular events [53], T1DM [54-56], diabetic nephropathy [57] }\end{array}$ \\
\hline hsa-miR-23a-3p & $19 \mathrm{p} 13.12$ & $\begin{array}{l}\text { Heart failure [58], coronary artery disease [59], cerebral ischemia-reperfusion [60], vascular endothelial dysfunction [15], } \\
\text { small and large abdominal aortic aneurysm [61], obesity and insulin resistance [62] }\end{array}$ \\
\hline hsa-miR-24-3p & $19 \mathrm{p} 13.12$ & $\begin{array}{l}\text { Asymptomatic carotid stenosis [63], familial hypercholesterolemia and coronary artery disease [64], angina pectoris [65], } \\
\text { ischemic dilated cardiomyopathy [66], small and large abdominal aortic aneurysm [61], myocardial ischemia/reperfusion } \\
\text { [67,68], diabetes mellitus [14,24,52,54] }\end{array}$ \\
\hline hsa-miR-26a-5p & $\begin{array}{c}3 \mathrm{p} 22.2 \\
12 \mathrm{q} 14.1[69]\end{array}$ & $\begin{array}{l}\text { Heart failure, cardiac hypertrophy [70], myocardial infarction [51,71,72], ischemia/reperfusion injury [73], pulmonary } \\
\text { arterial hypertension [74], T1DM [75], diabetic nephropathy [57] }\end{array}$ \\
\hline hsa-miR-29a-3p & $7 \mathrm{q} 32.3$ & $\begin{array}{c}\text { Ischemia/reperfusion-induced cardiac injury [76], cardiac cachexia, heart failure [77], atrial fibrillation [78], diffuse } \\
\text { myocardial fibrosis in hypertrophic cardiomyopathy [30], coronary artery disease [79], pulmonary arterial hypertension } \\
\text { [74], gestational diabetes mellitus [80], diabetes mellitus [13,23,81,82] }\end{array}$ \\
\hline hsa-miR-100-5p & $11 q 24.1$ & $\begin{array}{l}\text { Failing human heart, idiopathic dilated cardiomyopathy, ischemic cardiomyopathy [66], regulation of } \\
\text { hypertension-related genes [50], T1DM [54] }\end{array}$ \\
\hline
\end{tabular}


Table 1. Cont.

\begin{tabular}{|c|c|c|}
\hline miRBase ID & $\begin{array}{l}\text { Gene Location on } \\
\text { Chromosome }\end{array}$ & Role in the Pathogenesis of Cardiovascular/Cerebrovascular Diseases \\
\hline hsa-miR-103a-3p & $\begin{array}{c}5 \mathrm{q} 34 \\
20 \mathrm{p} 13[83]\end{array}$ & $\begin{array}{l}\text { Hypertension [84], hypoxia-induced pulmonary hypertension [85], myocardial ischemia/reperfusion injury, acute } \\
\text { myocardial infarction [84], ischemic dilated cardiomyopathy [66], obesity, regulation of insulin sensitivity [86], T1DM [87] }\end{array}$ \\
\hline hsa-miR-125b-5p & $\begin{array}{c}11 \mathrm{q} 24.1 \\
21 \mathrm{q} 21.1[88]\end{array}$ & $\begin{array}{l}\text { Acute ischemic stroke [89], acute myocardial infarction [90,91], ischemic dilated cardiomyopathy [66], ascending aortic } \\
\text { aneurysm [49], gestational diabetes mellitus [92], T1DM [93,94], T2DM [95] }\end{array}$ \\
\hline hsa-miR-126-3p & $9 q 34.3[96]$ & $\begin{array}{l}\text { Acute myocardial infarction [72], T2DM [53,97], T2DM with major cardiovascular events [53], gestational diabetes } \\
\text { mellitus [98] }\end{array}$ \\
\hline hsa-miR-133a-3p & $\begin{array}{c}18 \mathrm{q} 11.2 \\
20 \mathrm{q} 13.33[105]\end{array}$ & $\begin{array}{l}\text { Heart failure [106], myocardial fibrosis in hypertrophic cardiomyopathy }[30,107] \text {, arrhythmogenesis in the hypertrophic } \\
\text { and failing hearts }[108,109] \text {, coronary artery calcification [110], ascending aortic aneurysm [49], diabetes mellitus }[13,14]\end{array}$ \\
\hline hsa-miR-143-3p & $5 q 33$ & $\begin{array}{c}\text { Intracranial aneurysms [111], coronary heart disease [112], myocardial infarction [113], myocardial hypertrophy [114], } \\
\text { dilated cardiomyopathy [115], pulmonary arterial hypertension [116], acute ischemic stroke [89], ascending aortic } \\
\text { aneurysm [49] }\end{array}$ \\
\hline hsa-miR-145-5p & $5 q 33$ & $\begin{array}{l}\text { Hypertension [117,118], dilated cardiomyopathy [119], myocardial infarction [120,121], stroke [121], acute cerebral } \\
\text { ischemic/reperfusion [122], T2DM [24,123], T1DM [52], diabetic retinopathy [124], gestational diabetes mellitus [125] }\end{array}$ \\
\hline hsa-miR-146a-5p & $5 q 33.3[126,127]$ & $\begin{array}{l}\text { Angiogenesis [128], hypoxia, ischemia/reperfusion-induced cardiac injury [129], myocardial infarction [17], coronary } \\
\text { atherosclerosis, coronary heart disease in patients with subclinical hypothyroidism [130], acute ischemic stroke, acute } \\
\text { cerebral ischemia [131], T2DM [24,52], T1DM [75], diabetic nephropathy [57] }\end{array}$ \\
\hline hsa-miR-181a-5p & $\begin{array}{c}1 \mathrm{q} 32.1 \\
9 \mathrm{q} 33.3[132]\end{array}$ & $\begin{array}{l}\text { Regulation of hypertension-related genes [50], atherosclerosis [132], metabolic syndrome, coronary artery disease [133], } \\
\text { non-alcoholic fatty liver disease [134], ischaemic stroke, transient ischaemic attack, acute myocardial infarction [135,136], } \\
\text { obesity and insulin resistance [62,132,133], T1DM [52,137], T2DM [132,136] }\end{array}$ \\
\hline hsa-miR-195-5p & $17 \mathrm{p} 13.1[138]$ & $\begin{array}{l}\text { Cardiac hypertrophy, heart failure [139,140], abdominal aortic aneurysms [141], aortic stenosis [142], T2DM [123], } \\
\text { gestational diabetes mellitus [143] }\end{array}$ \\
\hline
\end{tabular}


Table 1. Cont

\begin{tabular}{|c|c|c|}
\hline miRBase ID & $\begin{array}{l}\text { Gene Location on } \\
\text { Chromosome }\end{array}$ & Role in the Pathogenesis of Cardiovascular/Cerebrovascular Diseases \\
\hline hsa-miR-199a-5p & $\begin{array}{c}1 \mathrm{q} 24.3 \\
19 \mathrm{p} 13.2\end{array}$ & $\begin{array}{c}\text { Hypertension [144], congenital heart disease [145], pulmonary artery hypertension [146], unstable angina [147], diabetic } \\
\text { retinopathy [148], T1DM, T2DM, gestational diabetes mellitus [149] }\end{array}$ \\
\hline hsa-miR-221-3p & Xp11.3 & $\begin{array}{c}\text { Asymptomatic carotid stenosis [63], cardiac amyloidosis [150], heart failure [151], atherosclerosis [152,153], aortic } \\
\text { stenosis [154], acute myocardial infarction [155], acute ischemic stroke [156], focal cerebral ischemia [157], pulmonary } \\
\text { artery hypertension [158], obesity [159] }\end{array}$ \\
\hline hsa-miR-342-3p & $14 \mathrm{q} 32.2$ & $\begin{array}{c}\text { Cardiac amyloidosis [150], obesity [160], T1DM [52,149,161], T2DM [149,162,163], GDM [149], endothelial } \\
\text { dysfunction [164] }\end{array}$ \\
\hline hsa-miR-499a-5p & $20 q 11.22$ & Myocardial infarction [17,165], hypoxia [166], cardiac regeneration [167], vascular endothelial dysfunction [15] \\
\hline hsa-miR-574-3p & 4 p14 & Myocardial infarction [168], coronary artery disease [100], cardiac amyloidosis [150], stroke [169], T2DM [104,170] \\
\hline
\end{tabular}




\section{Results}

MicroRNA gene expression was compared between mothers after normal and GDM-complicated pregnancies in maternal whole peripheral blood 3 to 11 years postpartum. Just the results, when the complicated cases reached a statistical significance, are presented below.

2.1. Expression Profile of MicroRNAs Associated with Diabetes Mellitus and Cardiovascular/Cerebrovascular Diseases in Mothers after GDM Pregnancies

The expression of miR-1-3p $(p<0.001)$, miR-16-5p $(p<0.001), \operatorname{miR}-17-5 p(p<0.001), \operatorname{miR}-20 a-5 p$ $(p<0.001), \operatorname{miR}-20 b-5 p(p<0.001), \operatorname{miR}-21-5 p(p<0.001), \operatorname{miR}-23 a-3 p(p<0.001), \operatorname{miR}-24-3 p(p=0.007)$, miR-26a-5p $(p<0.001)$, miR-29a-3p $(p<0.001), \operatorname{miR}-100-5 p(p<0.001), \operatorname{miR}-103 a-3 p(p<0.001)$, miR-125b-5p $(p<0.001)$, miR-126-3p $(p<0.001)$, miR-130b-3p $(p<0.001), \operatorname{miR}-133 a-3 p(p<0.001)$, miR-143-3p $(p<0.001), \operatorname{miR}-145-5 p(p=0.002), \operatorname{miR}-146 a-5 p(p<0.001), \operatorname{miR}-181 a-5 p(p<0.001)$, miR-195-5p $(p<0.001)$, miR-199a-5p $(p<0.001)$, miR-221-3p $(p<0.001)$, miR-342-3p $(p=0.003)$, miR-499a-5p $(p<0.001)$, and miR-574-3p $(p<0.001)$ differed significantly between the control group and women previously affected with GDM. These microRNAs were upregulated in GDM-affected patients (Figures 1 and 2).

The ROC curve analysis confirmed a significant upregulation of these particular microRNAs for mothers with a history of GDM when the comparison to the controls was performed (Figure 1).

The very good sensitivity at 10.0\% FPR for miR-1-3p (43.24\%), miR-16-5p (20.72\%), miR-17-5p (21.62\%), miR-20b-5p (38.74\%), miR-21-5p (28.83\%), miR-23a-3p (17.12\%), miR-26a-5p (20.72\%), miR-29a-3p (21.62\%), miR-103a-3p (30.63\%), miR-133a-3p (18.92\%), miR-146a-5p (17.12\%), miR-181a-5p (31.53\%), miR-195-5p (16.22\%), miR-199a-5p (15.32\%), miR-221-3p (31.53\%), and miR-499a-5p (28.83\%) is found (Figure 2).

However, despite the upregulation of microRNAs, the poor sensitivity at $10.0 \%$ FPR for miR-20a-5p (10.81\%), miR-24-3p (0.90\%), miR-100-5p (13.51\%), miR-125b-5p (9.91\%), miR-126-3p (9.91\%), miR-130b-3p (3.60\%), miR-143-3p (13.51\%), miR-145-5p (7.21\%), miR-342-3p (1.8\%), and miR-574-3p $(10.81 \%)$ was detected, therefore, these particular microRNAs were not further used for diabetes mellitus and cardiovascular risk assessment in mothers previously affected with GDM (Figure 2).

Combined screening of miR-1-3p, miR-16-5p, miR-17-5p, miR-20b-5p, miR-21-5p, miR-23a-3p, miR-26a-5p, miR-29a-3p, miR-103a-3p, miR-133a-3p, miR-146a-5p, miR-181a-5p, miR-195-5p, miR-199a-5p, miR-221-3p, and miR-499a-5p showed the highest accuracy for mothers with a prior exposure to GDM (AUC 0.900, $p<0.001$, sensitivity $77.48 \%$, specificity $93.26 \%$, cut off $>0.611270413$ ). It was able to identify $77.48 \%$ of mothers with an increased cardiovascular risk at $10.0 \%$ FPR (Figure 3 ). 


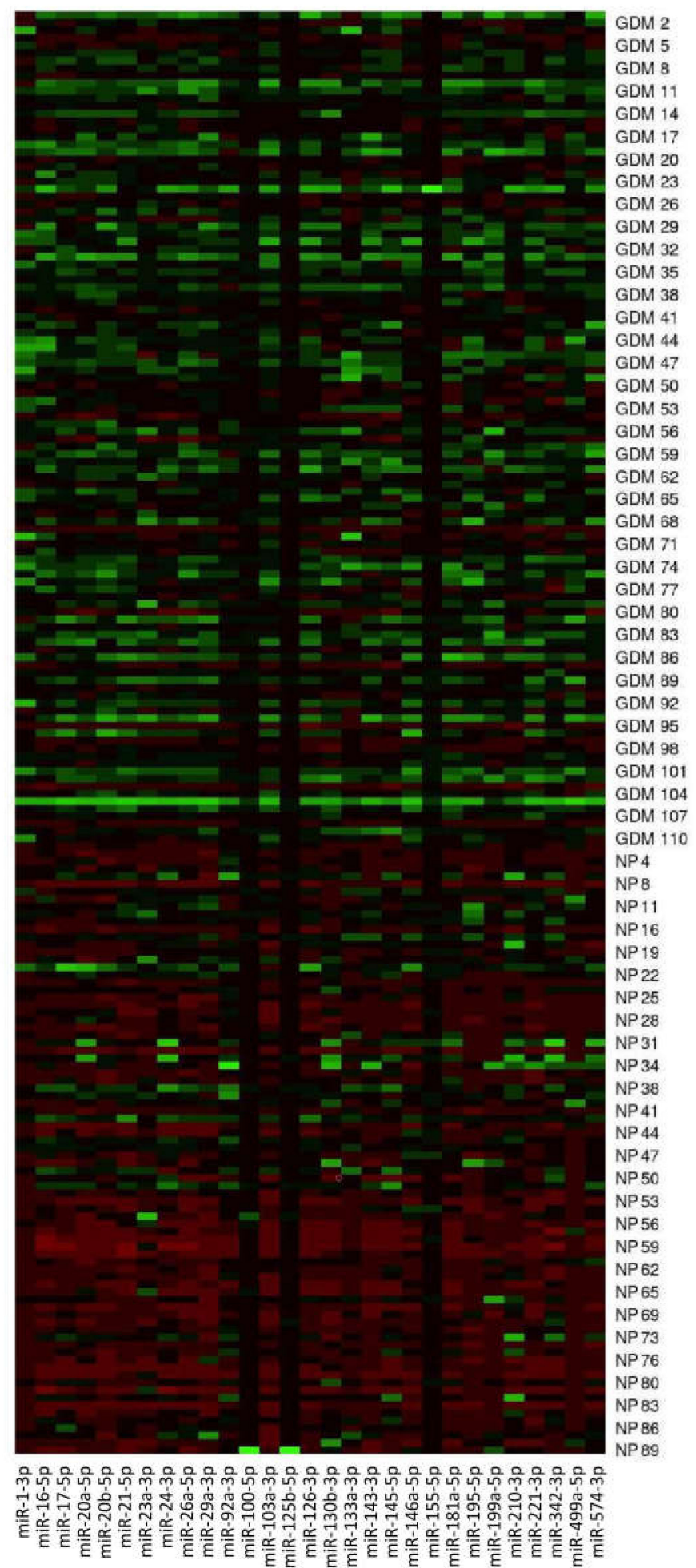

Figure 1. Postpartal microRNA expression profile in mothers with a history of GDM pregnancies. MicroRNA gene expression data $\left(2^{-\Delta \Delta \mathrm{Ct}}\right)$ are visualized using the heat map. In this setting, each row represents a sample (GDM1-GDM111, NP1-NP89) and each column represents a microRNA gene. The color and intensity of the boxes are used to represent changes of gene expression $\left(2^{-\Delta \Delta \mathrm{Ct}}\right)$. Green color indicates upregulation, and red color indicates downregulation. NP, normal pregnancies. 
A

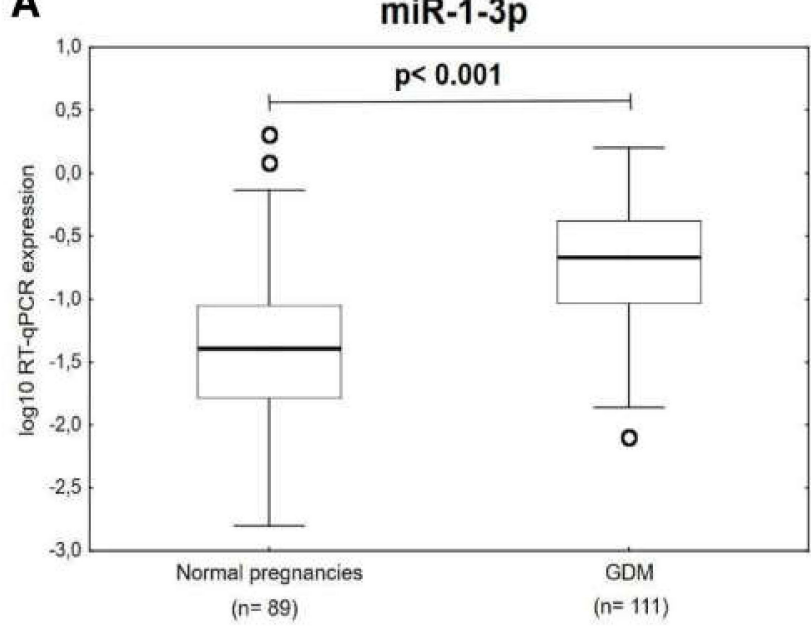

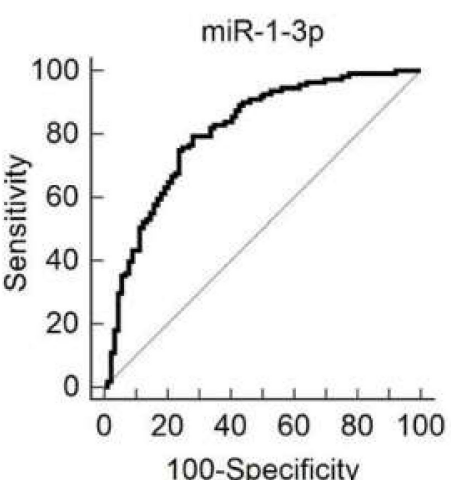

100-Specificity

\begin{tabular}{|l|r|}
\hline Area under the ROC curve (AUC) & 0.809 \\
\hline
\end{tabular}

\begin{tabular}{|l|r|}
\hline Standard Error & 0.0314 \\
\hline
\end{tabular}

\begin{tabular}{l|l}
$95 \%$ Confidence interval & 0.747 to 0.861
\end{tabular}

\begin{tabular}{l|r|}
\hline Significance level P (Area=0.5) & $<0.0001$ \\
\hline
\end{tabular}

Estimated sensitivity at fixed specificity

\begin{tabular}{l|l|l|l} 
Specificity & Sensitivity & $95 \% \mathrm{Cl}$ & Criterion
\end{tabular}

\begin{tabular}{|l|c|c|c|}
\hline 90.00 & 43.24 & 27.03 to 62.16 & $>0.243781644$ \\
\hline
\end{tabular}

\begin{tabular}{|l|r|r|r|r|r|r|r|r|}
\hline Criterion & Sensitivity & $95 \% \mathrm{Cl}$ & Specificity & $95 \% \mathrm{Cl}$ & $+\mathrm{LR}$ & $95 \% \mathrm{Cl}$ & $-\mathrm{LR}$ & $95 \% \mathrm{Cl}$ \\
\hline$>0.07707102$ & 79.28 & $70.5-86.4$ & 71.91 & $61.4-80.9$ & 2.82 & $2.0-4.0$ & 0.29 & $0.2-0.4$ \\
\hline
\end{tabular}

B

miR-16-5p

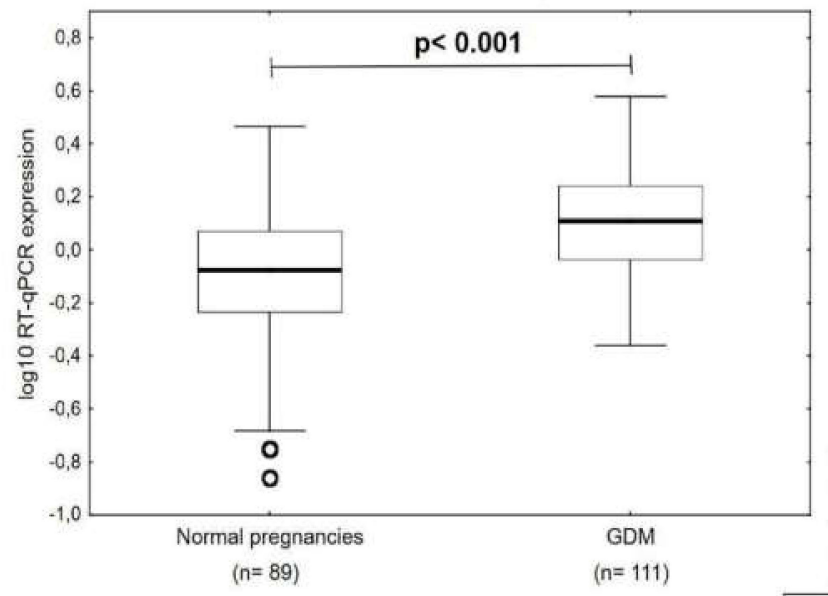

miR-16-5p

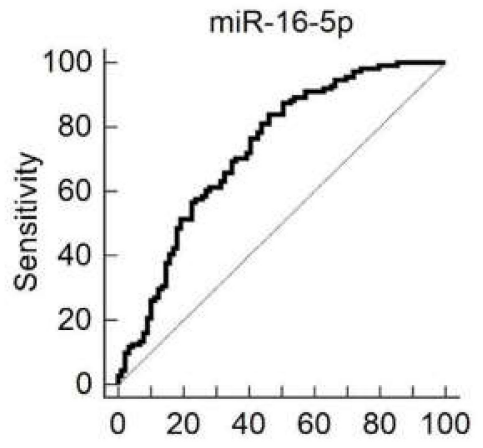

100-Specificity

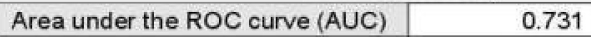

\begin{tabular}{|l|r|}
\hline Standard Error & 0.0364 \\
\hline
\end{tabular}

$95 \%$ Confidence interval $\quad 0.664$ to 0.792

\begin{tabular}{lr}
\hline Significance level P (Area $=0.5$ ) & $<0.0001$
\end{tabular}

Estimated sensitivity at fixed specificity

\begin{tabular}{l|l|l|l} 
Specificity & Sensitivity & $95 \% \mathrm{Cl}$ & Criterion
\end{tabular}

\begin{tabular}{|l|c|c|c|}
\hline 90.00 & 20.72 & 7.91 to 40.54 & $>1.811273587$ \\
\hline
\end{tabular}

\begin{tabular}{|l|r|r|r|r|r|r|r|r|}
\hline Criterion & Sensitivity & $95 \% \mathrm{Cl}$ & Specificity & $95 \% \mathrm{Cl}$ & $+\mathrm{LR}$ & $95 \% \mathrm{Cl}$ & $-\mathrm{LR}$ & $95 \% \mathrm{Cl}$ \\
\hline$>0.847047662$ & 83.78 & $75.6-90.1$ & 53.93 & $43.0-64.6$ & 1.82 & $1.4-2.3$ & 0.30 & $0.2-0.5$ \\
\hline
\end{tabular}

Figure 2. Cont. 
C

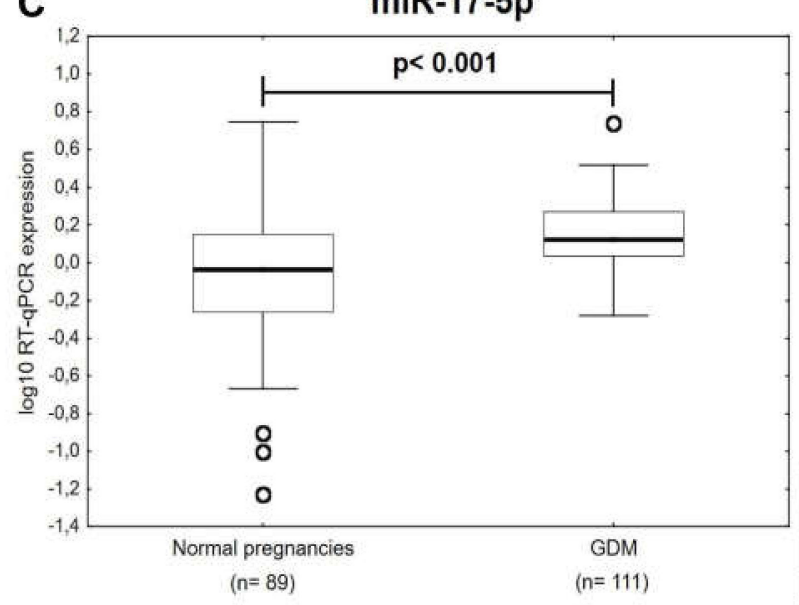

miR-17-5p

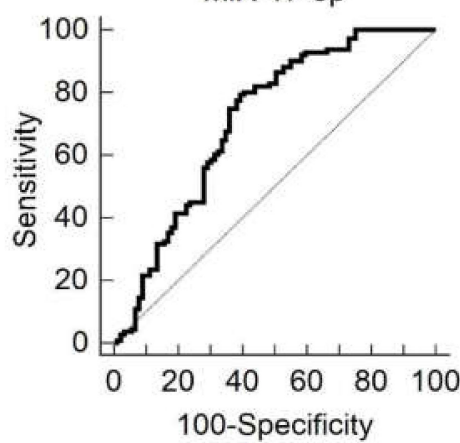

100-Specificity

\begin{tabular}{|l|r|}
\hline Area under the ROC curve (AUC) & 0.712 \\
\hline
\end{tabular} \begin{tabular}{|l|l}
\hline Standard Error & 0.0382 \\
\hline
\end{tabular} \begin{tabular}{l|l}
$95 \%$ Confidence interval & 0.644 to 0.774
\end{tabular} Significance level P (Area $=0.5$ ) $<0,0001$

Estimated sensitivity at fixed specificity \begin{tabular}{l|l|l|}
\hline Specificity & Sensitivity & $95 \% \mathrm{Cl}$ \\
\hline
\end{tabular} \begin{tabular}{|l|c|c|c|}
\hline 90.00 & 21.62 & 4.50 to 41.44 & $>1.978599453$ \\
\hline
\end{tabular}

\begin{tabular}{|l|r|r|r|r|r|r|r|r|}
\hline Criterion & Sensitivity & $95 \% \mathrm{Cl}$ & Specificity & $95 \% \mathrm{Cl}$ & +LR & $95 \% \mathrm{Cl}$ & $-\mathrm{LR}$ & $95 \% \mathrm{Cl}$ \\
\hline$>1.035235049$ & 79.28 & $70.5-86.4$ & 60.67 & $49.7-70.9$ & 2.02 & $1.5-2.7$ & 0.34 & $0.2-0.5$ \\
\hline
\end{tabular}

\section{D} miR-20a-5p

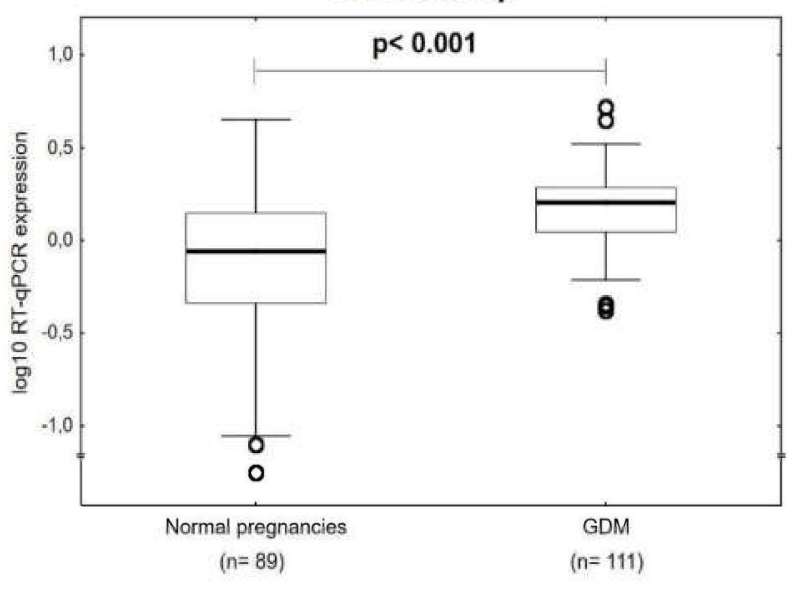

Figure 2. Cont. 
E
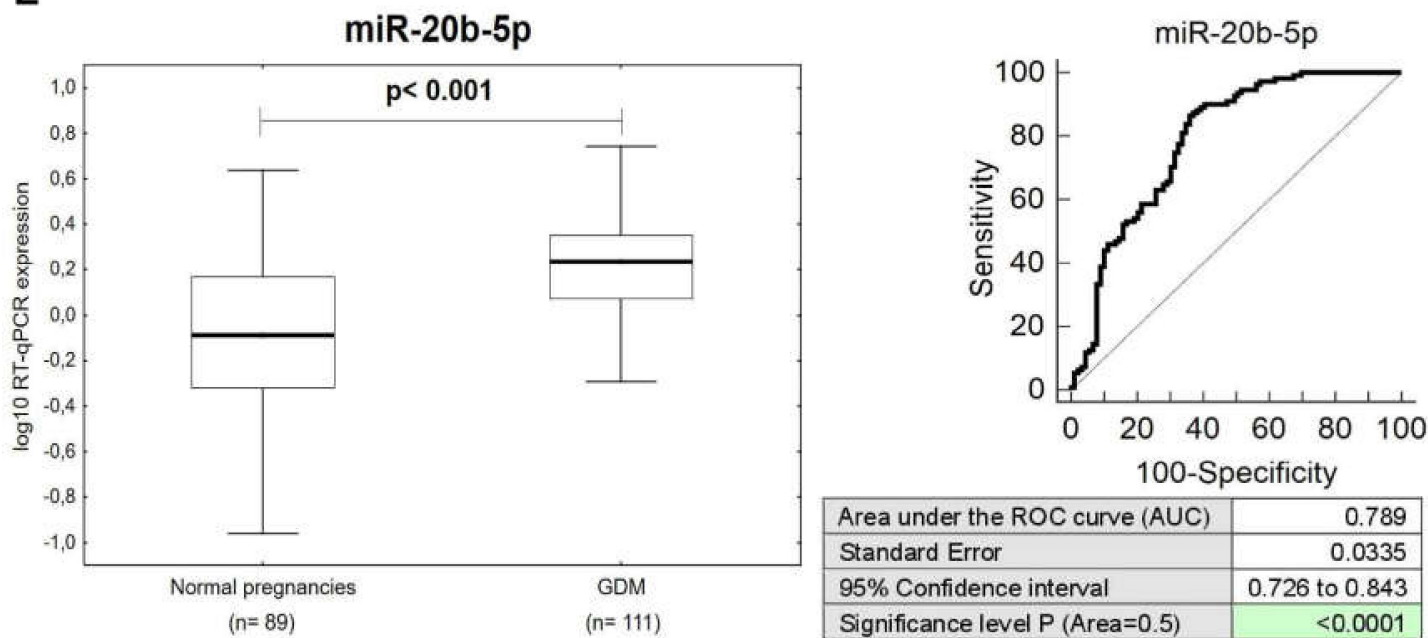

\begin{tabular}{|l|r|}
\hline Area under the ROC curve (AUC) & 0.789 \\
\hline Standard Error & 0.0335 \\
\hline $95 \%$ Confidence interval & 0.726 to 0.843 \\
\hline Significance level P (Area $=0.5$ ) & $<0.0001$ \\
\hline
\end{tabular}

Estimated sensitivity at fixed specificity

\begin{tabular}{|l|l|l|l} 
Specificity & Sensitivity & $95 \% \mathrm{Cl}$ & Criterion \\
\hline
\end{tabular}

\begin{tabular}{|l|c|c|c|}
\hline 90.00 & 38.74 & 7.21 to 54.05 & $>2.031561229$ \\
\hline
\end{tabular}

\begin{tabular}{|l|r|r|r|r|r|r|r|r|}
\hline Criterion & Sensitivity & $95 \% \mathrm{Cl}$ & Specificity & $95 \% \mathrm{Cl}$ & $+\mathrm{LR}$ & $95 \% \mathrm{Cl}$ & $-\mathrm{LR}$ & $95 \% \mathrm{Cl}$ \\
\hline$>1.06610565$ & 86.49 & $78.7-92.2$ & 64.04 & $53.2-73.9$ & 2.41 & $1.8-3.2$ & 0.21 & $0.1-0.3$ \\
\hline
\end{tabular}

\section{F}

miR-21-5p
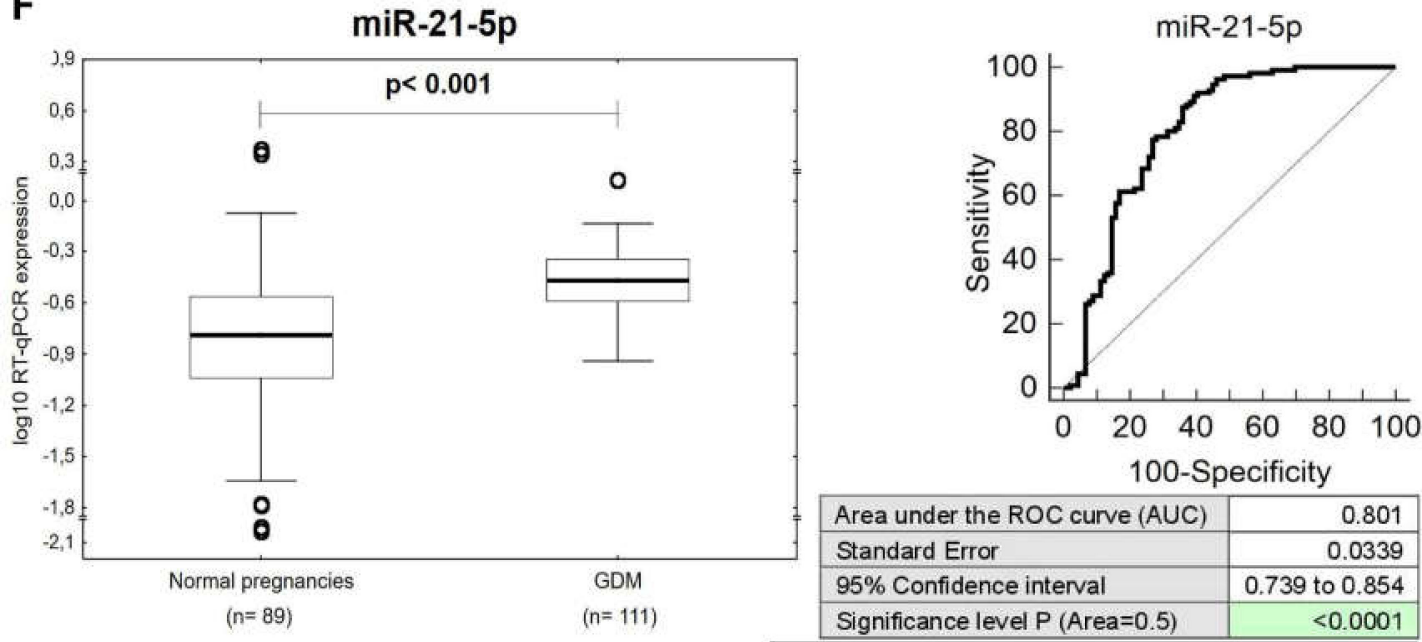

\begin{tabular}{|l|r|}
\hline Area under the ROC curve (AUC) & 0.801 \\
\hline Standard Error & 0.0339 \\
\hline $95 \%$ Confidence interval & 0.739 to 0.854 \\
\hline Significance level P (Area $=0.5$ ) & $<0.0001$ \\
\hline
\end{tabular}

Estimated sensitivity at fixed specificity

\begin{tabular}{|c|c|c|c|} 
Specificity & Sensitivity & $95 \% \mathrm{Cl}^{\text {a }}$ & Criterion \\
\hline
\end{tabular}

\begin{tabular}{|l|c|c|c|}
90.00 & 28.83 & 1.80 to 58.56 & $>0.4168634$ \\
\hline
\end{tabular}

\begin{tabular}{|l|r|r|r|r|r|r|r|r|}
\hline Criterion & Sensitivity & $95 \% \mathrm{Cl}$ & Specificity & $95 \% \mathrm{Cl}$ & $+\mathrm{LR}$ & $95 \% \mathrm{Cl}$ & $-\mathrm{LR}$ & $95 \% \mathrm{Cl}$ \\
\hline$>0.203612$ & 90.99 & $84.1-95.6$ & 60.67 & $49.7-70.9$ & 2.31 & $1.8-3.0$ & 0.15 & $0.08-0.3$ \\
\hline
\end{tabular}

Figure 2. Cont. 
G

miR-23a-3p

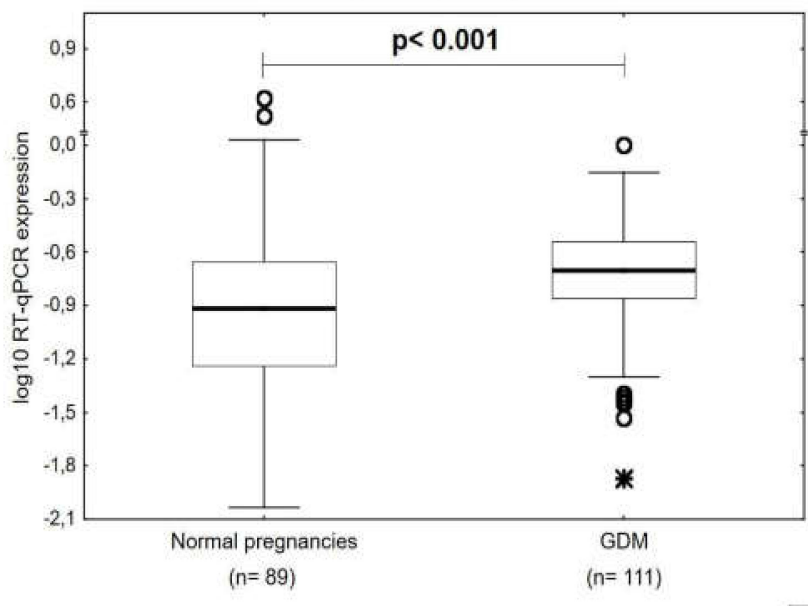

miR-23a-3p

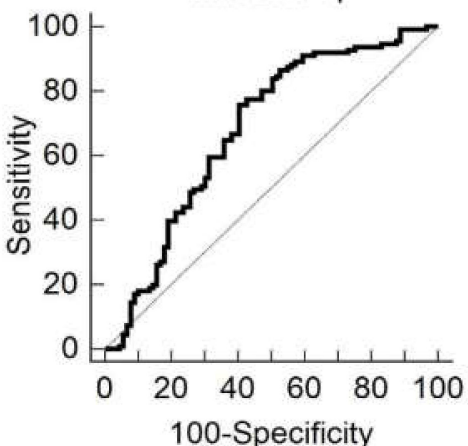

100-Specificity

\begin{tabular}{|l|r|}
\hline Area under the ROC curve (AUC) & 0.678 \\
\hline
\end{tabular}

Standard Error $\quad 0.0396$

\begin{tabular}{l|l}
\hline $95 \%$ Confidence interval & 0.608 to 0.742 \\
\hline
\end{tabular}

\begin{tabular}{ll} 
Significance level P (Area $=0.5)$ & $<0.0001$ \\
\hline
\end{tabular}

Estimated sensitivity at fixed specificity

\begin{tabular}{l|c|c|c|} 
Specificity & Sensitivity & $95 \% \mathrm{Cl}$ & Criterion \\
\hline
\end{tabular}

\begin{tabular}{l|c|c|c|}
\hline 90.00 & 17.12 & 2.70 to 35.14 & $>0.35543583$ \\
\hline
\end{tabular}

\begin{tabular}{|l|r|r|r|r|r|r|r|r|}
\hline Criterion & Sensitivity & $95 \% \mathrm{Cl}$ & Specificity & $95 \% \mathrm{Cl}$ & $+\mathrm{LR}$ & $95 \% \mathrm{Cl}$ & $-\mathrm{LR}$ & $95 \% \mathrm{Cl}$ \\
\hline$>0.138429628$ & 75.68 & $66.6-83.3$ & 59.55 & $48.6-69.8$ & 1.87 & $1.4-2.5$ & 0.41 & $0.3-0.6$ \\
\hline
\end{tabular}

H

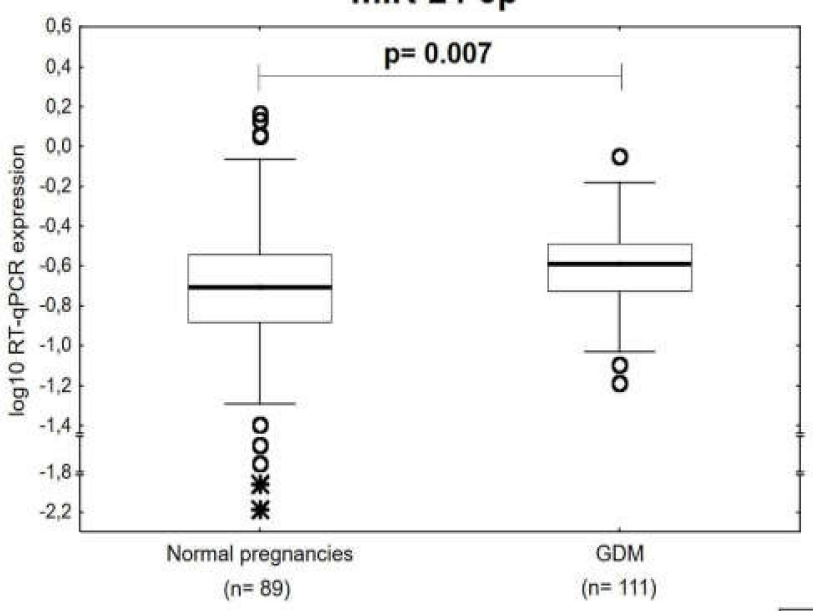

miR-24-3p

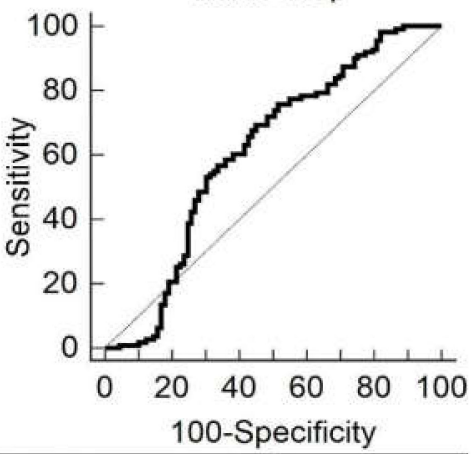

100-Specificity

\begin{tabular}{|l|r|}
\hline Area under the ROC curve (AUC) & 0.611 \\
\hline Standard Error & 0.0421 \\
\hline $95 \%$ Confidence interval & 0.540 to 0.679 \\
\hline Significance level P (Area=0.5) & 0.0084 \\
\hline
\end{tabular}
0.0084

Estimated sensitivity at fixed specificity

\begin{tabular}{l|l|l|l} 
Specificity & Sensitivity & $95 \% \mathrm{Cl}$ & Criterion
\end{tabular} \begin{tabular}{|c|c|c|c|}
\hline 90.00 & 0.90 & 0.00 to 3.60 & $>0.663051646$ \\
\hline
\end{tabular}

\begin{tabular}{|l|r|r|r|r|r|r|r|r|}
\hline Criterion & Sensitivity & $95 \% \mathrm{Cl}$ & Specificity & $95 \% \mathrm{Cl}$ & $+\mathrm{LR}$ & $95 \% \mathrm{Cl}$ & $-\mathrm{LR}$ & $95 \% \mathrm{Cl}$ \\
\hline$>0.206367886$ & 69.37 & $59.9-77.8$ & 55.06 & $44.1-65.6$ & 1.54 & $1.2-2.0$ & 0.56 & $0.4-0.8$ \\
\hline
\end{tabular}

Figure 2. Cont. 
I

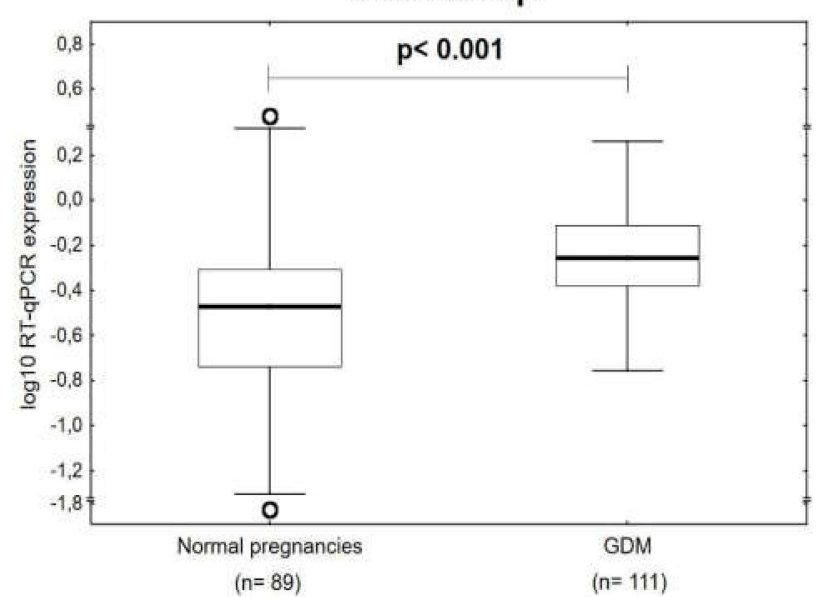

miR-26a-5p

\begin{tabular}{|c|c|c|c|c|c|c|c|c|c|}
\hline \multirow{2}{*}{\multicolumn{10}{|c|}{ Estimated sensitivity at fixed specificity }} \\
\hline & & & & & & & & & \\
\hline & & & \multicolumn{2}{|c|}{ Specificity } & \multicolumn{2}{|c|}{ Sensitivity } & $95 \% \mathrm{Cl}$ & \multicolumn{2}{|r|}{ Criterion } \\
\hline & & & \multicolumn{2}{|c|}{90.00} & \multicolumn{2}{|c|}{20.72} & 0.00 to 45.95 & \multicolumn{2}{|c|}{$>0.813409286$} \\
\hline Criterion & Sensitivity & $95 \% \mathrm{Cl}$ & Specificity & & $95 \% \mathrm{Cl}$ & + LR & $95 \% \mathrm{Cl}$ & $-L R$ & $95 \% \mathrm{Cl}$ \\
\hline$>0.30533702$ & 90.99 & $84.1-95.6$ & 49.44 & 38.7 & -60.2 & 1.80 & $1.5-2.2$ & 0.18 & $0.10-0.3$ \\
\hline
\end{tabular}

miR-26a-5p

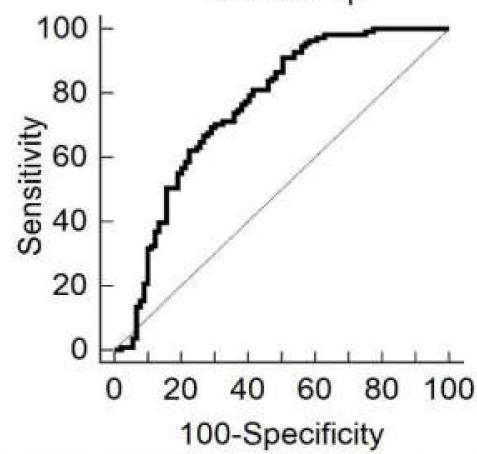

Area under the ROC curve (AUC)

Standard Error

95\% Confidence interva

0.814

0001

J

miR-29a-3p
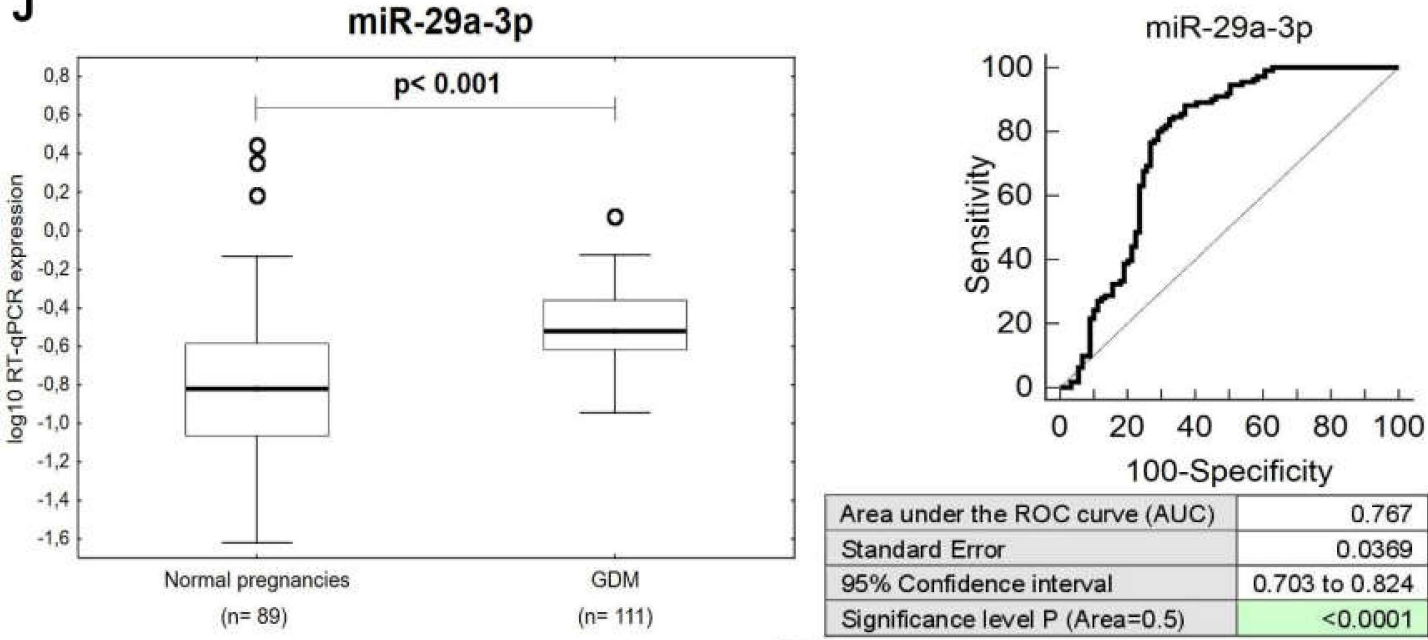

100-Specificity

\begin{tabular}{|l|r|}
\hline Area under the ROC curve (AUC) & 0.767 \\
\hline
\end{tabular}

\begin{tabular}{lr} 
Standard Error & 0.0369 \\
\hline
\end{tabular}

\begin{tabular}{l|l}
$95 \%$ Confidence interval & 0.703 to 0.824
\end{tabular}

Significance level P (Area $=0.5$ ) $\quad<0.0001$

Estimated sensitivity at fixed specificity

\begin{tabular}{|l|l|l|l}
\hline Specificity & Sensitivity & $95 \% \mathrm{Cl}$ & Criterion \\
\hline
\end{tabular}

\begin{tabular}{|l|c|c|c|}
\hline 90.00 & 21.62 & 0.90 to 35.14 & $>0.452747201$ \\
\hline
\end{tabular}

\begin{tabular}{|l|l|l|l|l|l|l|l|r|}
\hline Criterion & Sensitivity & $95 \% \mathrm{Cl}$ & Specificity & $95 \% \mathrm{Cl}$ & $+\mathrm{LR}$ & $95 \% \mathrm{Cl}$ & $-\mathrm{LR}$ & $95 \% \mathrm{Cl}$ \\
\hline
\end{tabular}

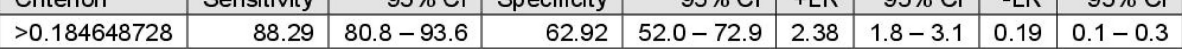

Figure 2. Cont. 
K

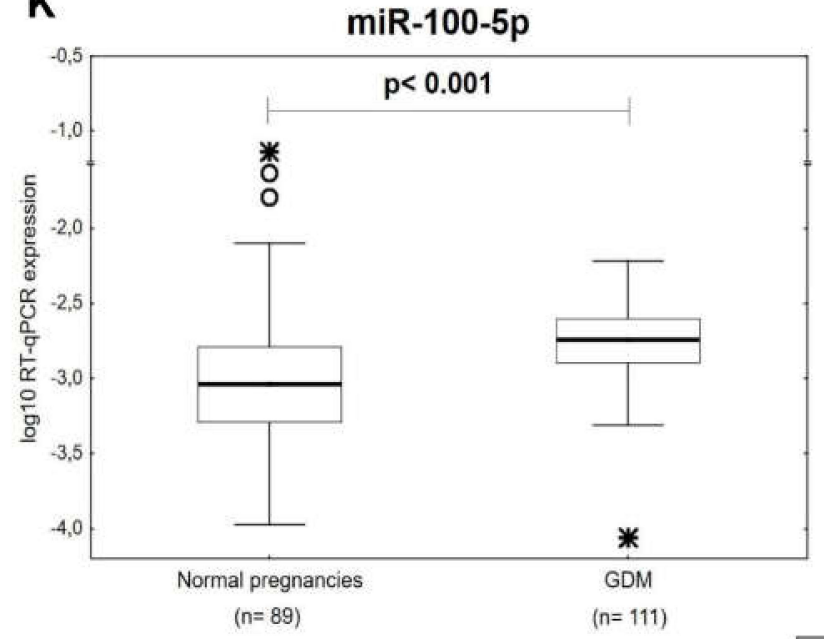

miR-100-5p

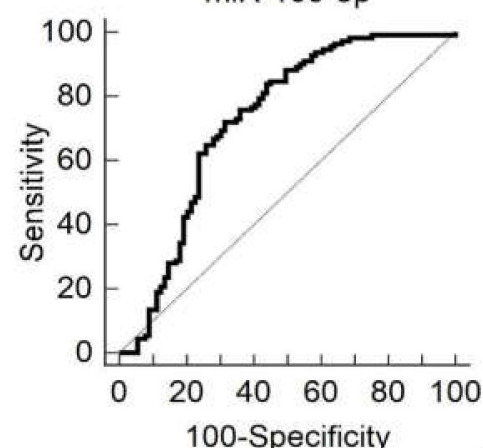

100-Specificity

\begin{tabular}{|r|r|}
\hline Area under the ROC curve (AUC) & 0.726 \\
\hline
\end{tabular}

Standard Error

95\% Confidence interval

0.0384

\begin{tabular}{l|l}
0.659 to 0.787 \\
\hline
\end{tabular}

Estimated sensitivity at fixed specificity

\begin{tabular}{|c|c|c|c|} 
Specificity & Sensitivity & $95 \% \mathrm{Cl}$ & Criterion \\
\hline
\end{tabular}

\begin{tabular}{l|c|c|c|}
\hline 90.00 & 13.51 & 0.00 to 36.04 & $>0.003245268$ \\
\hline
\end{tabular}

\begin{tabular}{|l|r|r|r|r|r|r|r|r|}
\hline Criterion & Sensitivity & $95 \% \mathrm{Cl}$ & Specificity & $95 \% \mathrm{Cl}$ & $+\mathrm{LR}$ & $95 \% \mathrm{Cl}$ & $-\mathrm{LR}$ & $95 \% \mathrm{Cl}$ \\
\hline$>0.001373692$ & 72.07 & $62.8-80.2$ & 68.54 & $57.8-78.0$ & 2.29 & $1.7-3.2$ & 0.41 & $0.3-0.6$ \\
\hline
\end{tabular}

L

miR-103a-3p
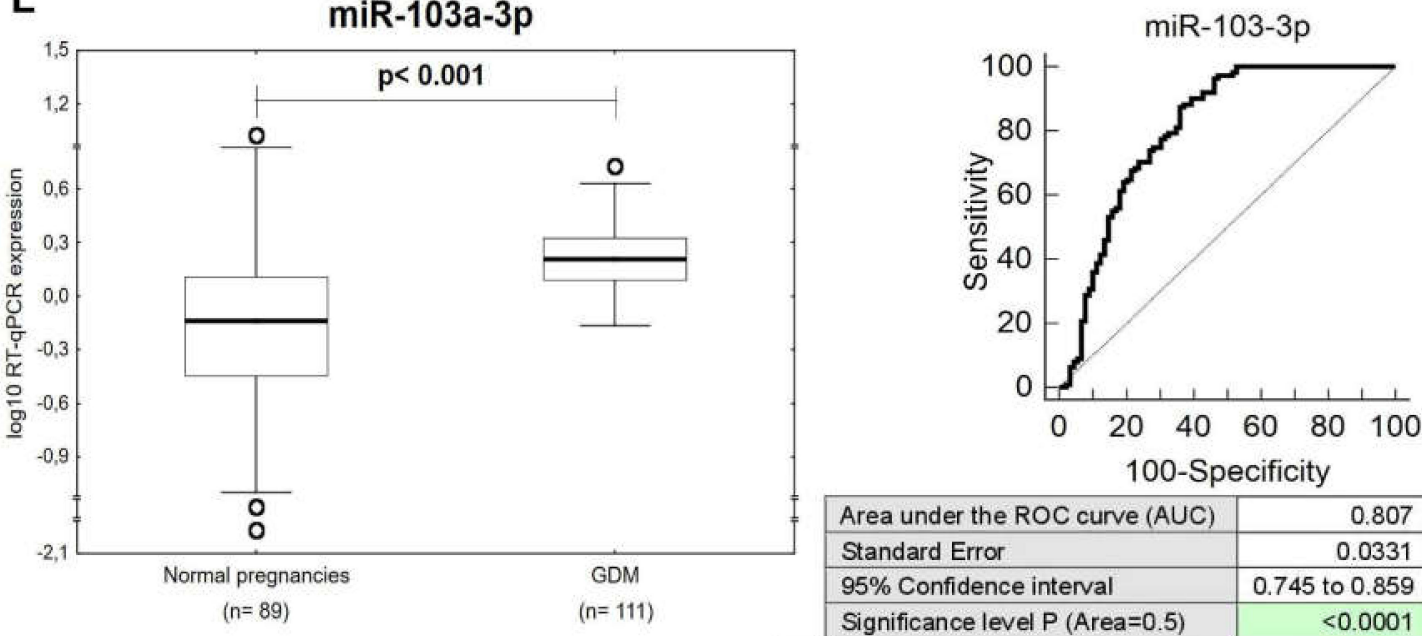

100-Specificity

\begin{tabular}{|l|r|}
\hline Area under the ROC curve (AUC) & 0.807 \\
\hline
\end{tabular}

\begin{tabular}{l|r} 
Standard Error & 0.0331
\end{tabular}

\begin{tabular}{l|l}
$95 \%$ Confidence interval & 0.745 to 0.859
\end{tabular}

$\begin{array}{ll}\text { Significance level P (Area }=0.5 \text { ) } & <0.0001\end{array}$

Estimated sensitivity at fixed specificity

\begin{tabular}{l|l|l|l} 
Specificity & Sensitivity & $95 \% \mathrm{Cl}$ & Criterion
\end{tabular}

\begin{tabular}{|l|c|c|c|}
\hline 90.00 & 30.63 & 2.80 to 54.05 & $>1.921479885$ \\
\hline
\end{tabular}

\begin{tabular}{|l|r|r|r|r|r|r|r|r|}
\hline Criterion & Sensitivity & $95 \% \mathrm{Cl}$ & Specificity & $95 \% \mathrm{Cl}$ & $+\mathrm{LR}$ & $95 \% \mathrm{Cl}$ & $-\mathrm{LR}$ & $95 \% \mathrm{Cl}$ \\
\hline$>1.050663852$ & 87.39 & $79.7-92.9$ & 64.04 & $53.2-73.9$ & 2.43 & $1.8-3.2$ & 0.20 & $0.1-0.3$ \\
\hline
\end{tabular}

Figure 2. Cont. 
M

miR-125b-5p

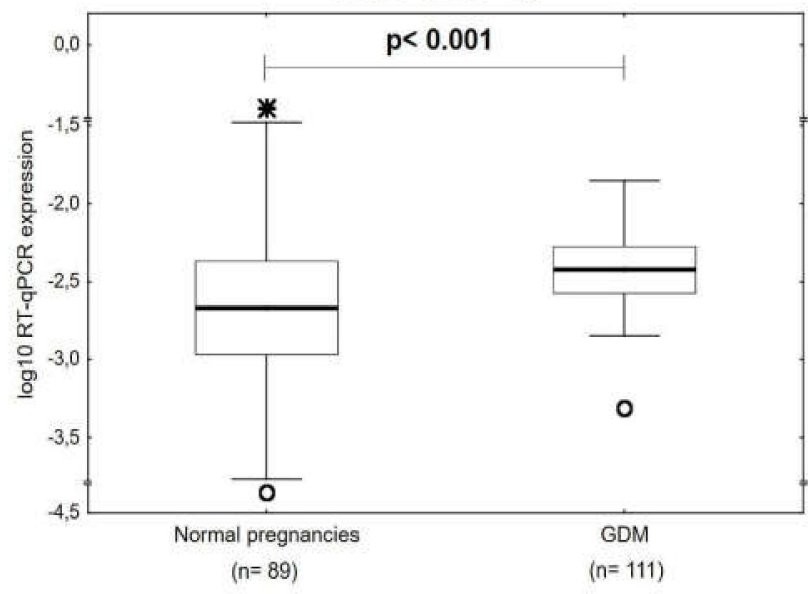

miR-125b-5p

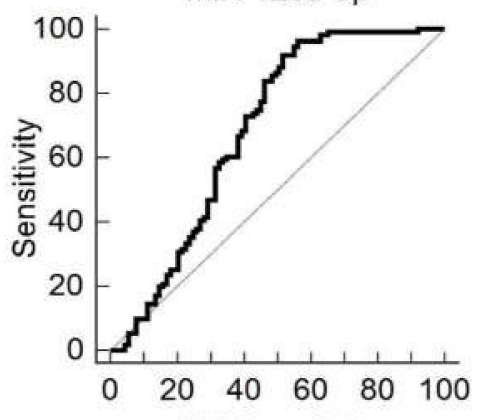

100-Specificity

\begin{tabular}{|l|r|}
\hline Area under the ROC curve (AUC) & 0.685 \\
\hline
\end{tabular}

\begin{tabular}{l|r} 
Standard Error & 0.0406 \\
\hline
\end{tabular}

\begin{tabular}{l|l}
$95 \%$ Confidence interval & 0.616 to 0.749
\end{tabular}

Significance level P (Area $=0.5$ ) $\quad<0.0001$

Estimated sensitivity at fixed specificity

\begin{tabular}{|l|l|l|l} 
Specificity & Sensitivity & $95 \% \mathrm{Cl}$ & Criterion
\end{tabular}

\begin{tabular}{|l|l|l|l|}
\hline 90.00 & 9.91 & 0.90 to 24.32 & $>0.006963705$ \\
\hline
\end{tabular}

\begin{tabular}{|l|r|r|r|r|r|r|r|r|}
\hline Criterion & Sensitivity & $95 \% \mathrm{Cl}$ & Specificity & $95 \% \mathrm{Cl}$ & $+\mathrm{LR}$ & $95 \% \mathrm{Cl}$ & $-\mathrm{LR}$ & $95 \% \mathrm{Cl}$ \\
\hline$>0.001716706$ & 96.40 & $91.0-99.0$ & 43.82 & $33.3-54.7$ & 1.72 & $1.4-2.1$ & 0.082 & $0.03-0.2$ \\
\hline
\end{tabular}

$\mathbf{N}$

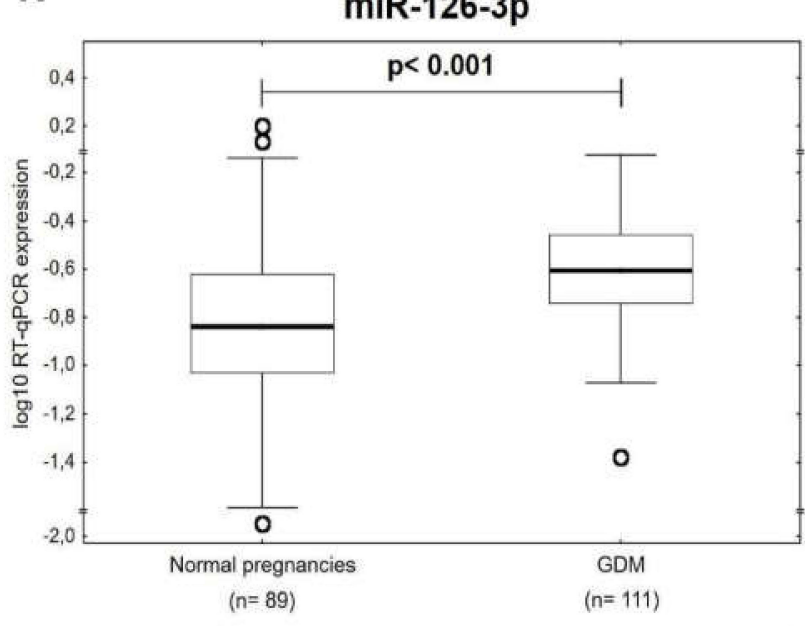

miR-126-3p

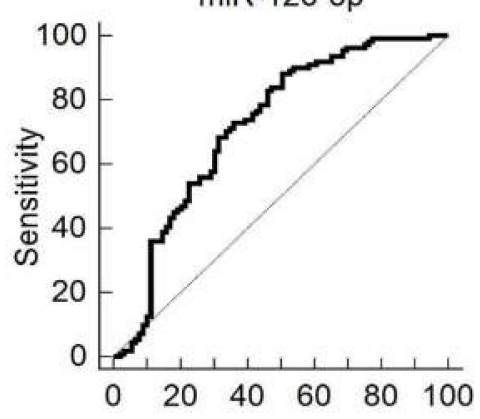

100-Specificity

\begin{tabular}{|l|r|}
\hline Area under the ROC curve (AUC) & 0.721 \\
\hline Standard Error & 0.0378 \\
\hline 95\% Confidence interval & 0.653 to 0.782 \\
\hline Significance level P (Area $=0.5$ ) & $<0.0001$ \\
\hline
\end{tabular}

Estimated sensitivity at fixed specificity \begin{tabular}{|l|c|c|c|}
\hline Specificity & Sensitivity & $95 \% \mathrm{Cl}$ & Criterion \\
\hline
\end{tabular} \begin{tabular}{|l|c|c|c|}
\hline 90.00 & 9.91 & 0.90 to 43.24 & $>0.463658697$ \\
\hline
\end{tabular}

\begin{tabular}{|l|r|r|r|r|r|r|r|r|}
\hline Criterion & Sensitivity & $95 \% \mathrm{Cl}$ & Specificity & $95 \% \mathrm{Cl}$ & $+\mathrm{LR}$ & $95 \% \mathrm{Cl}$ & $-\mathrm{LR}$ & $95 \% \mathrm{Cl}$ \\
\hline$>0.137792568$ & 88.29 & $80.8-93.6$ & 49.44 & $38.7-60.2$ & 1.75 & $1.4-2.2$ & 0.24 & $0.1-0.4$ \\
\hline
\end{tabular}

Figure 2. Cont. 
0

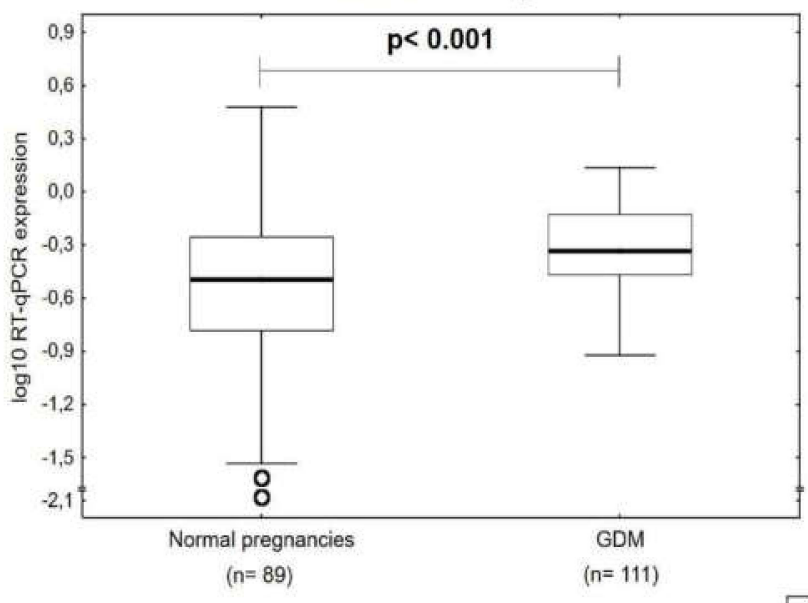

miR-130b-3p

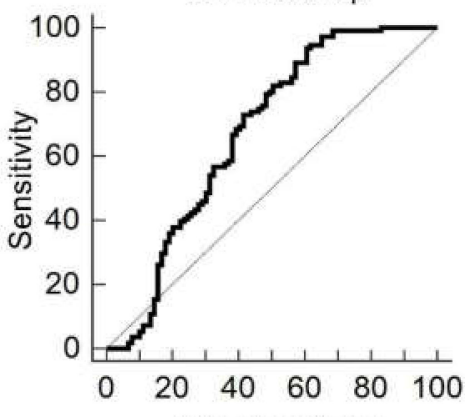

100-Specificity

\begin{tabular}{|l|r|}
\hline Area under the ROC curve (AUC) & 0.671 \\
\hline Standard Error & 0.0407 \\
\hline 95\% Confidence interval & 0.601 to 0.736 \\
\hline Significance level P (Area=0.5) & $<0.0001$ \\
\hline
\end{tabular}

Significance level P (Area $=0.5$ )

Estimated sensitivity at fixed specificity

\begin{tabular}{l|l|l|l}
\hline Specificity & Sensitivity & $95 \% \mathrm{Cl}$ & Criterion \\
\hline
\end{tabular}

\begin{tabular}{|l|c|c|c|}
\hline 90.00 & 3.60 & 0.00 to 16.22 & $>1.222296607$ \\
\hline
\end{tabular}

\begin{tabular}{|l|r|r|r|r|r|r|r|r|}
\hline Criterion & Sensitivity & $95 \% \mathrm{Cl}$ & Specificity & $95 \% \mathrm{Cl}$ & $+\mathrm{LR}$ & $95 \% \mathrm{Cl}$ & $-\mathrm{LR}$ & $95 \% \mathrm{Cl}$ \\
\hline$>0.237338536$ & 93.69 & $87.4-97.4$ & 39.33 & $29.1-50.3$ & 1.54 & $1.3-1.8$ & 0.16 & $0.07-0.3$ \\
\hline
\end{tabular}

$\mathbf{P}$

miR-133a-3p

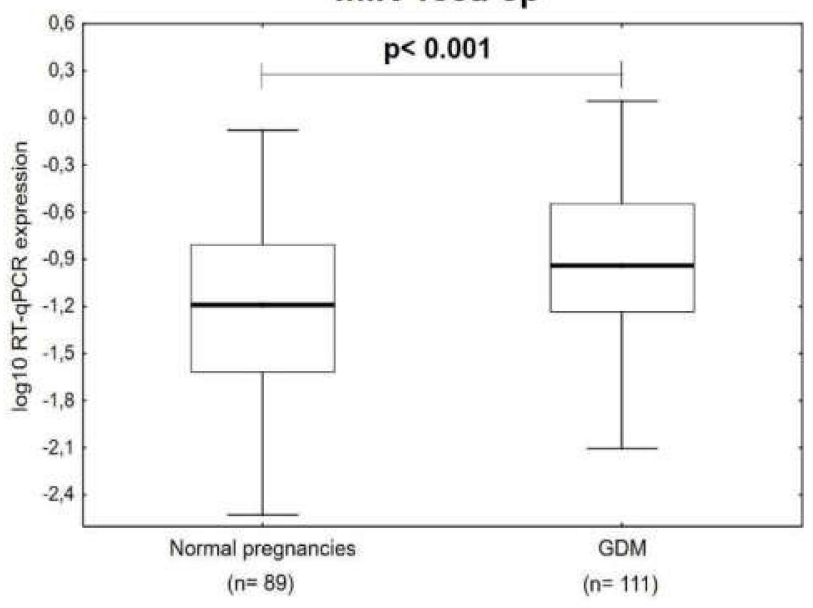

miR-133a-3p

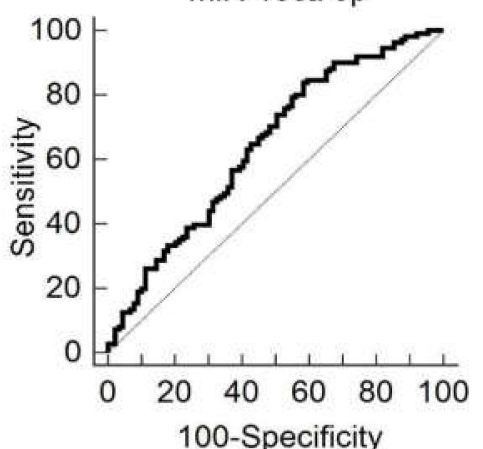

100-Specificity

\begin{tabular}{|l|r|}
\hline Area under the ROC curve (AUC) & 0.642 \\
\hline Standard Error & 0.0396 \\
\hline
\end{tabular}

\begin{tabular}{l|r} 
Standard Error & 0.0396 \\
\hline $95 \%$ Confidence interval & 0.572 to 0.709
\end{tabular}

\begin{tabular}{ll}
\hline Significance level P (Area $=0.5$ ) & 0.0003 \\
\hline
\end{tabular}

Estimated sensitivity at fixed specificity

\begin{tabular}{|l|c|c|c|}
\hline Specificity & Sensitivity & $95 \% \mathrm{Cl}$ & Criterion \\
\hline
\end{tabular}

\begin{tabular}{|l|c|c|c|}
\hline 90.00 & 18.92 & 8.46 to 34.23 & $>0.339958635$ \\
\hline
\end{tabular}

\begin{tabular}{|l|r|r|r|r|r|r|r|r|}
\hline Criterion & Sensitivity & $95 \% \mathrm{Cl}$ & Specificity & $95 \% \mathrm{Cl}$ & $+\mathrm{LR}$ & $95 \% \mathrm{Cl}$ & $-\mathrm{LR}$ & $95 \% \mathrm{Cl}$ \\
\hline$>0.043814829$ & 83.78 & $75.6-90.1$ & 41.57 & $31.2-52.5$ & 1.43 & $1.2-1.7$ & 0.39 & $0.2-0.6$ \\
\hline
\end{tabular}

Figure 2. Cont. 
Q

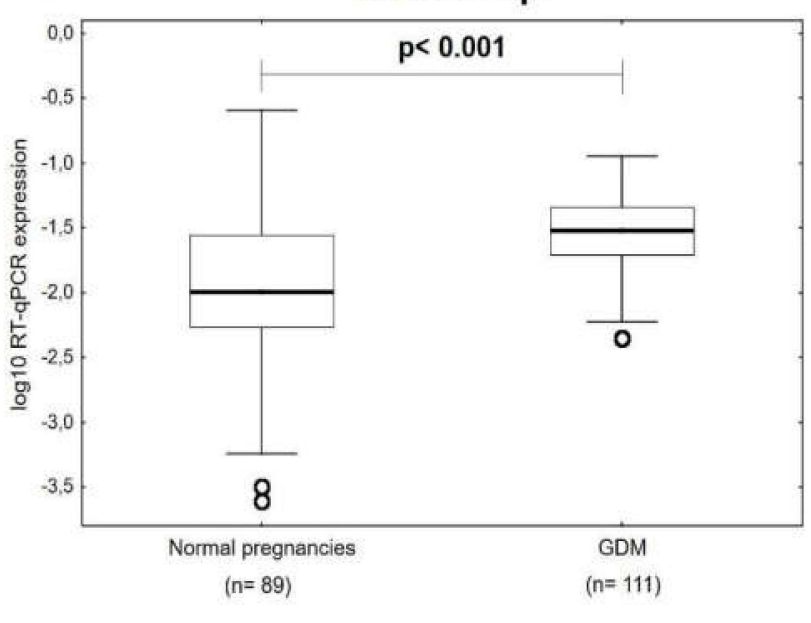

miR-143-3p

\begin{tabular}{|c|c|c|c|c|c|c|c|c|}
\hline & & & \multicolumn{6}{|c|}{ Estimated sensitivity at fixed specificity } \\
\hline & & & Specific & \begin{tabular}{l|l} 
city & Sensitiv \\
\end{tabular} & & $95 \% \mathrm{Cl}$ & & iterion \\
\hline & & & 90.00 & 13.51 & & .00 to 28.83 & $>0.05$ & 58609791 \\
\hline Colier & Sensitivity & $95 \% \mathrm{Cl}$ & Specificity & $95 \% \mathrm{Cl}$ & $+\mathrm{LR}$ & $95 \% \mathrm{Cl}$ & -LR & $95 \% \mathrm{Cl}$ \\
\hline$>0.015321578$ & 85.59 & $77.6-91.5$ & 62.92 & $52.0-72.9$ & 2.31 & $1.7-3.1$ & 0.23 & $0.1-0.4$ \\
\hline
\end{tabular}

R

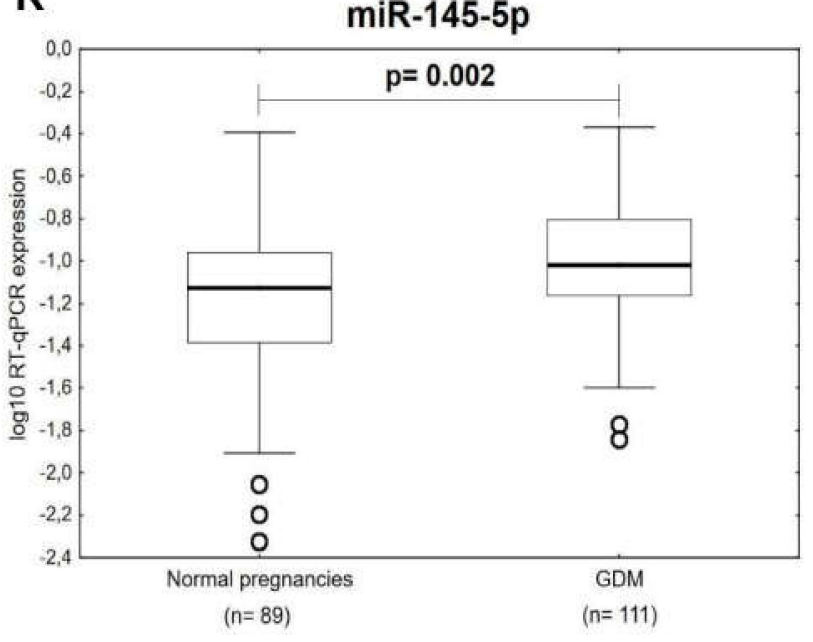

miR-145-5p

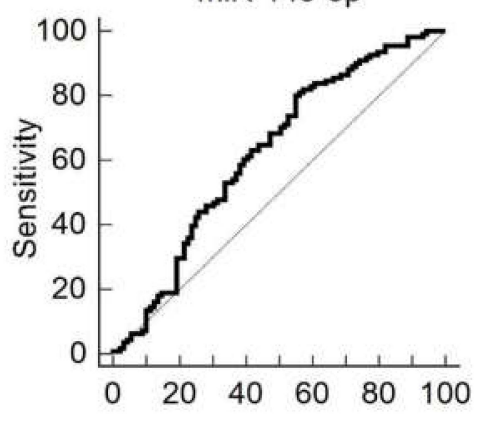

100-Specificity

\begin{tabular}{|l|r|}
\hline Area under the ROC curve (AUC) & 0.626 \\
\hline Standard Error & 0.0407 \\
\hline $95 \%$ Confidence interval & 0.555 to 0.693 \\
\hline Significance level P (Area=0.5) & 0.0019 \\
\hline
\end{tabular}

Estimated sensitivity at fixed specificity

\begin{tabular}{|c|c|c|c|} 
Specificity & Sensitivity & $95 \% \mathrm{Cl}$ & Criterion \\
\hline
\end{tabular}

\begin{tabular}{|l|c|c|c|}
\hline 90.00 & 7.21 & 0.90 to 18.02 & $>0.203564228$ \\
\hline
\end{tabular}

\begin{tabular}{|l|r|r|r|r|r|r|r|r|}
\hline Criterion & Sensitivity & $95 \% \mathrm{Cl}$ & Specificity & $95 \% \mathrm{Cl}$ & $+\mathrm{LR}$ & $95 \% \mathrm{Cl}$ & $-\mathrm{LR}$ & $95 \% \mathrm{Cl}$ \\
\hline$>0.062462943$ & 80.18 & $71.5-87.1$ & 44.94 & $34.4-55.9$ & 1.46 & $1.2-1.8$ & 0.44 & $0.3-0.7$ \\
\hline
\end{tabular}

Figure 2. Cont. 
S

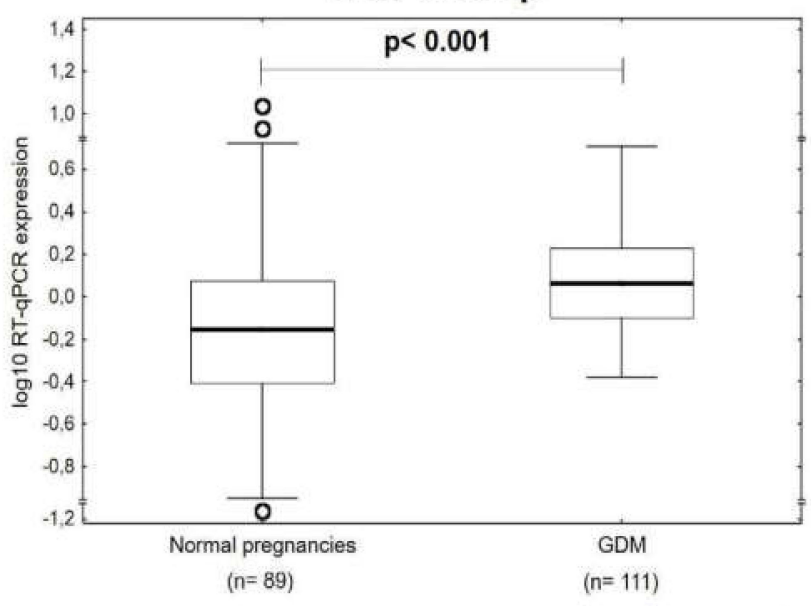

miR-146a-5p

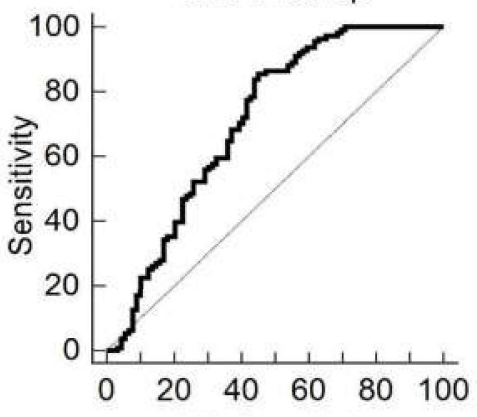

100-Specificity

\begin{tabular}{|l|r|}
\hline Area under the ROC curve (AUC) & 0.710 \\
\hline
\end{tabular}

Standard Error $\quad 0.0385$

\begin{tabular}{l|l}
\hline $95 \%$ Confidence interval & 0.642 to 0.772
\end{tabular}

\begin{tabular}{l|l} 
Significance level P (Area $=0.5$ ) & $<0.0001$ \\
\hline
\end{tabular}

Estimated sensitivity at fixed specificity

\begin{tabular}{|l|l|l|l}
\hline Specificity & Sensitivity & $95 \% \mathrm{Cl}$ & Criterion \\
\hline
\end{tabular}

\begin{tabular}{|l|c|c|c|}
\hline 90.00 & 17.12 & 0.90 to 31.77 & $>2.016985101$ \\
\hline
\end{tabular}

\begin{tabular}{|l|r|r|r|r|r|r|r|r|}
\hline Criterion & Sensitivity & $95 \% \mathrm{Cl}$ & Specificity & $95 \% \mathrm{Cl}$ & $+\mathrm{LR}$ & $95 \% \mathrm{Cl}$ & $-\mathrm{LR}$ & $95 \% \mathrm{Cl}$ \\
\hline$>0.719213841$ & 85.59 & $77.6-91.5$ & 55.06 & 44.65 .6 & 1.90 & $1.5-2.4$ & 0.26 & $0.2-0.4$ \\
\hline
\end{tabular}

T

miR-181a-5p
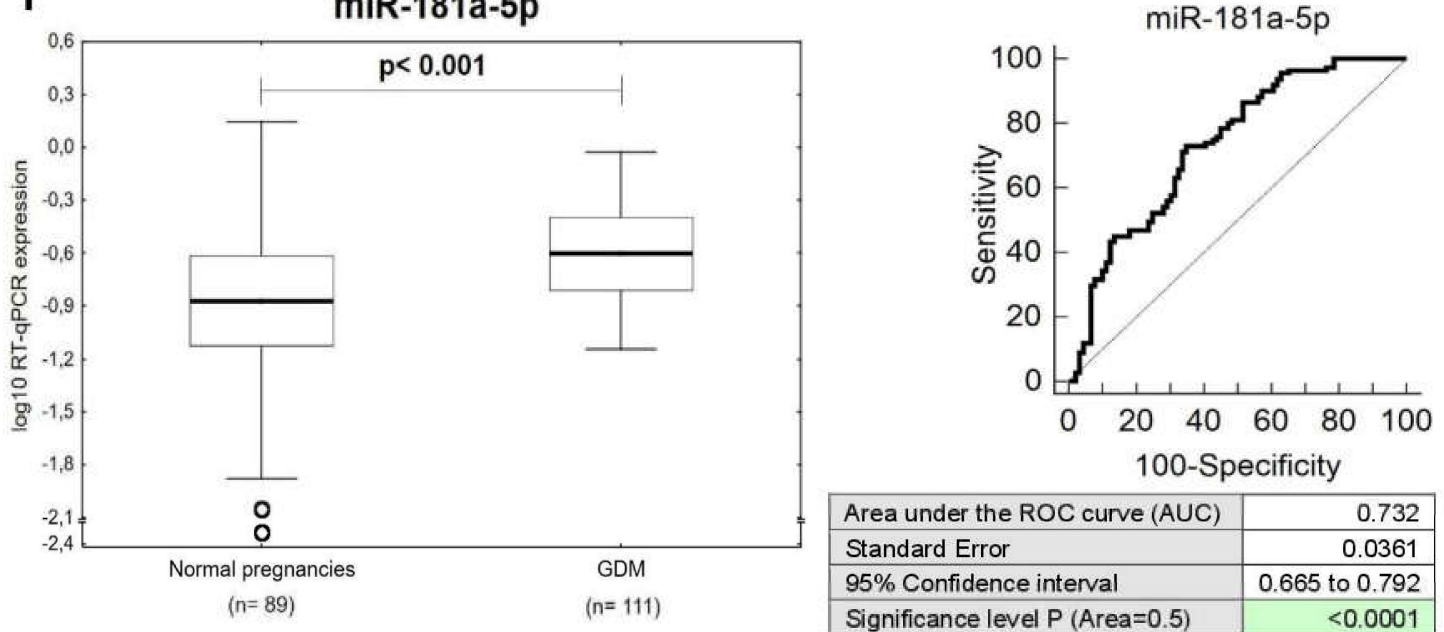

\begin{tabular}{|l|r|}
\hline Area under the ROC curve (AUC) & 0.732 \\
\hline
\end{tabular}

\begin{tabular}{l|r} 
Standard Error & 0.0361 \\
\hline
\end{tabular}

\begin{tabular}{l|l}
$95 \%$ Confidence interval & 0.665 to 0.792
\end{tabular}

Significance level P (Area $=0.5$ $<0.0001$

Estimated sensitivity at fixed specificity

\begin{tabular}{l|l|l|l} 
Specificity & Sensitivity & $95 \% \mathrm{Cl}$ & Criterion
\end{tabular}

\begin{tabular}{|l|c|c|c|}
\hline 90.00 & 31.53 & 4.50 to 46.85 & $>0.341828696$ \\
\hline
\end{tabular}

\begin{tabular}{|l|r|r|r|r|r|r|r|r|}
\hline Criterion & Sensitivity & $95 \% \mathrm{Cl}$ & Specificity & $95 \% \mathrm{Cl}$ & $+\mathrm{LR}$ & $95 \% \mathrm{Cl}$ & $-\mathrm{LR}$ & $95 \% \mathrm{Cl}$ \\
\hline$>0.179703784$ & 72.97 & $63.7-81.0$ & 65.17 & $54.3-75.0$ & 2.10 & $1.5-2.8$ & 0.41 & $0.3-0.6$ \\
\hline
\end{tabular}

Figure 2. Cont. 
U

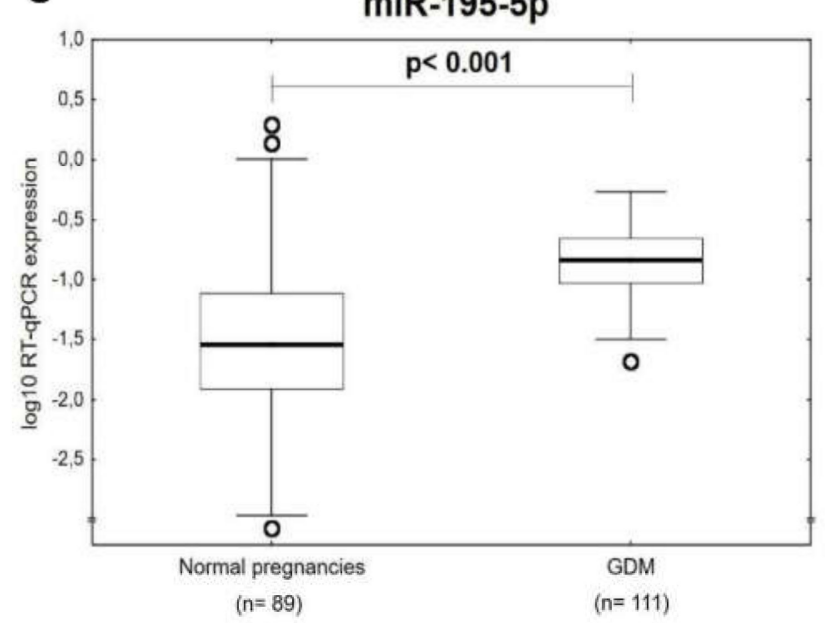

miR-195-5p

\begin{tabular}{|c|c|c|c|c|c|c|}
\hline \multicolumn{7}{|c|}{ Estimated sensitivity at fixed specificity } \\
\hline \multicolumn{2}{|c|}{ Specificity } & \multicolumn{2}{|c|}{ Sensitivity } & $95 \% \mathrm{Cl}$ & \multicolumn{2}{|r|}{ Criterion } \\
\hline 90.00 & & 16.2 & & 0.90 to 66.67 & & .245660227 \\
\hline sificity & & $95 \% \mathrm{Cl}$ & $+\mathrm{LR}$ & $95 \% \mathrm{Cl}$ & $-L R$ & $95 \% \mathrm{Cl}$ \\
\hline 70.79 & 60.2 & $2-79.9$ & 3.08 & \begin{tabular}{l|l}
8 & $2.2-4.3$ \\
\end{tabular} & 0.14 & $0.08-0.2$ \\
\hline
\end{tabular}

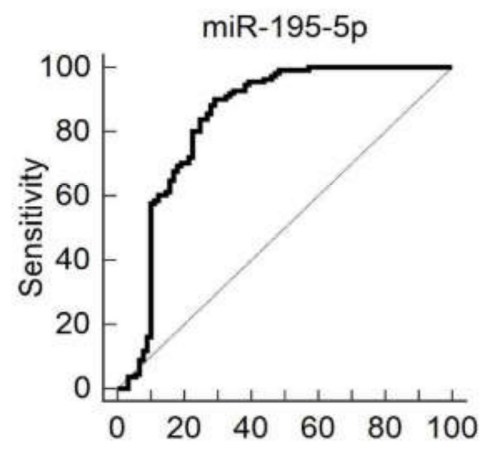

100-Specificity

\begin{tabular}{|l|r|}
\hline Area under the ROC curve (AUC) & 0.837 \\
\hline Standard Error & 0.0323 \\
\hline $95 \%$ Confidence interval & 0.779 to 0.886 \\
\hline Significance level P (Area=0.5) & $<0.0001$ \\
\hline
\end{tabular}

. 7

\begin{tabular}{|l|r|r|r|r|r|r|r|r|}
\hline Criterion & Sensitivity & $95 \% \mathrm{Cl}$ & Specificity & $95 \% \mathrm{Cl}$ & $+\mathrm{LR}$ & $95 \% \mathrm{Cl}$ & $-\mathrm{LR}$ & $95 \% \mathrm{Cl}$ \\
\hline$>0.057571652$ & 90.09 & $83.0-94.9$ & 70.79 & $60.2-79.9$ & 3.08 & $2.2-4.3$ & 0.14 & $0.08-0.2$ \\
\hline
\end{tabular}

\section{v}

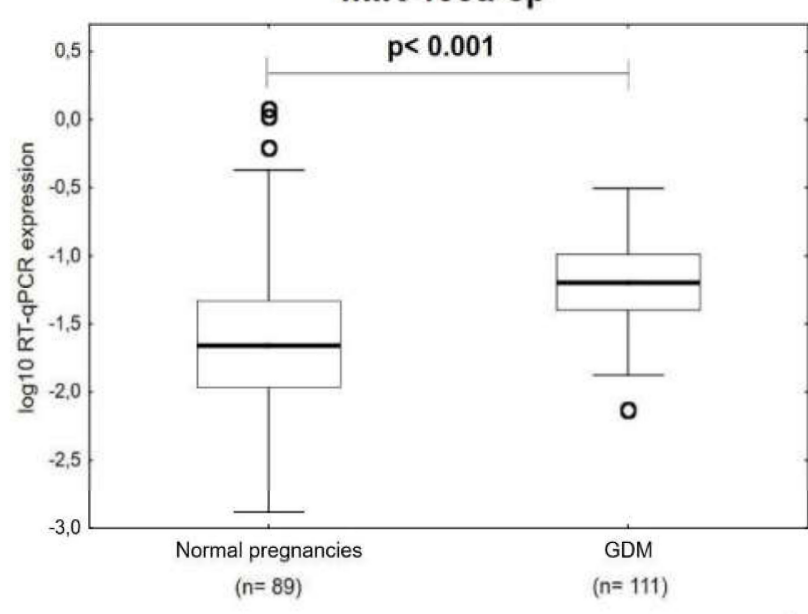

miR-199a-5p

\begin{tabular}{|c|c|c|c|c|c|c|c|c|c|}
\hline & & & \multirow{2}{*}{\multicolumn{7}{|c|}{ Estimated sensitivity at fixed specificity }} \\
\hline & & & & & & & & & \\
\hline & & & \multicolumn{2}{|c|}{ Specificity } & \multicolumn{2}{|c|}{ Sensitivity } & $95 \% \mathrm{Cl}$ & \multicolumn{2}{|c|}{ Criterion } \\
\hline & & & \multicolumn{2}{|l|}{90.00} & \multicolumn{2}{|l|}{15.32} & 0.00 to 46.85 & \multicolumn{2}{|c|}{$>0.130601067$} \\
\hline Criterion & Sensitivity & $95 \% \mathrm{Cl}$ & Specificity & & $95 \% \mathrm{Cl}$ & $+\mathrm{LR}$ & $95 \% \mathrm{Cl}$ & -LR & $95 \% \mathrm{Cl}$ \\
\hline$>0.03337978$ & 84.68 & $76.6-90.8$ & 65.17 & 54. & $3-75.0$ & 2.43 & \begin{tabular}{l|l}
3 & $1.8-3.3$
\end{tabular} & 0.24 & $0.1-0.4$ \\
\hline
\end{tabular}

Figure 2. Cont. 

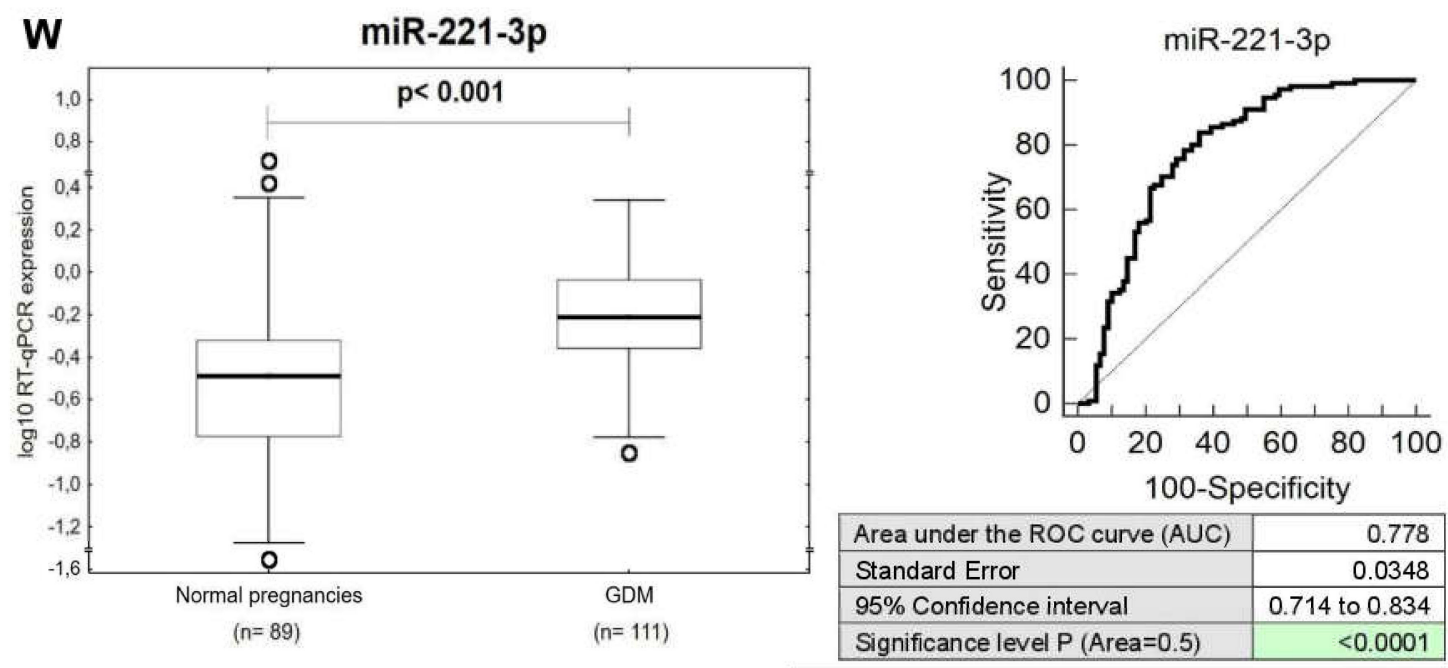

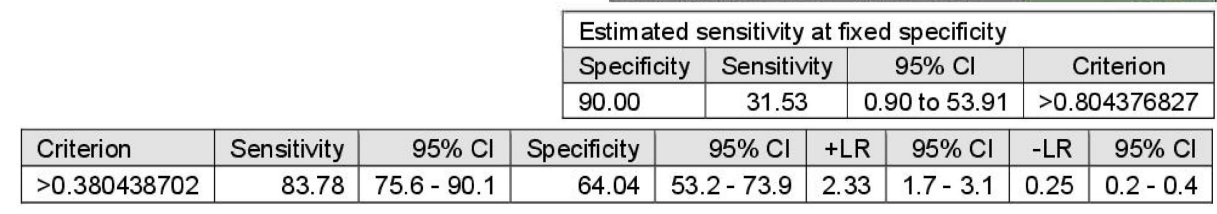

$\mathbf{X}$

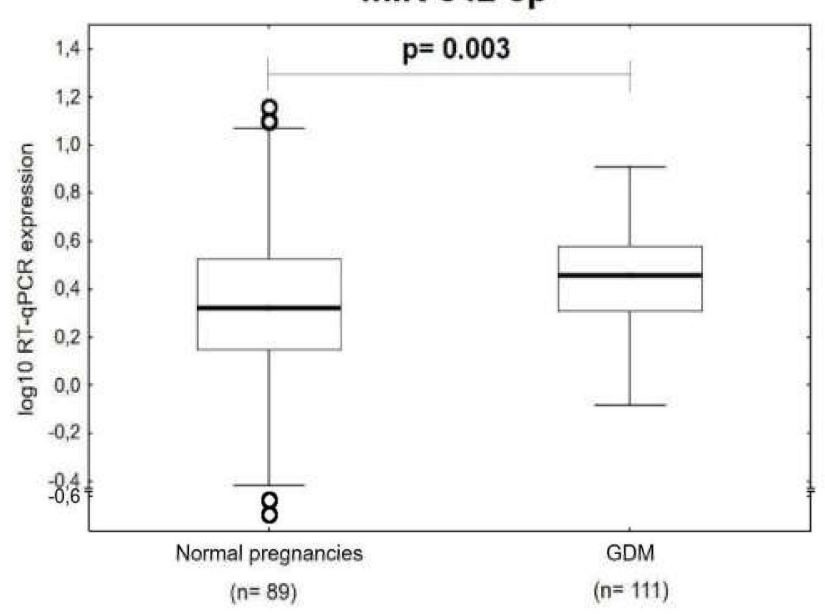

miR-342-3p

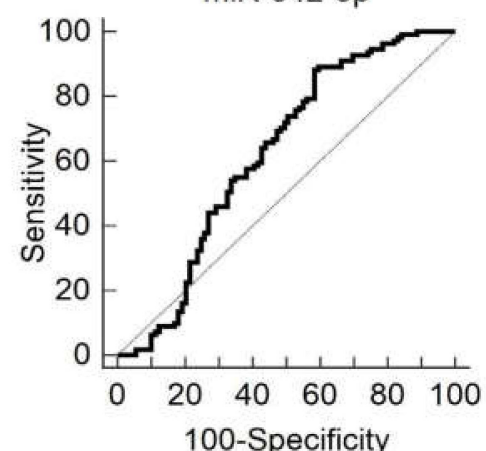

100-Specificity

\begin{tabular}{|l|r|}
\hline Area under the ROC curve (AUC) & 0.623 \\
\hline Standard Error & 0.0419 \\
\hline $95 \%$ Confidence interval & 0.552 to 0.691 \\
\hline Significance level P (Area=0.5) & 0.0032 \\
\hline
\end{tabular}

Estimated sensitivity at fixed specificity \begin{tabular}{l|l|l|l} 
Specificity & Sensitivity & $95 \% \mathrm{Cl}$ & Criterion \\
\hline
\end{tabular} \begin{tabular}{|l|c|c|c|}
\hline 90.00 & 1.80 & 0.00 to 9.01 & $>5.411758464$ \\
\hline
\end{tabular}

\begin{tabular}{|l|r|r|r|r|r|r|r|r|}
\hline Criterion & Sensitivity & $95 \% \mathrm{Cl}$ & Specificity & $95 \% \mathrm{Cl}$ & $+\mathrm{LR}$ & $95 \% \mathrm{Cl}$ & $-\mathrm{LR}$ & $95 \% \mathrm{Cl}$ \\
\hline$>1.82186452$ & 88.29 & $80.8-93.6$ & 41.57 & $31.2-52.5$ & 1.51 & $1.3-1.8$ & 0.28 & $0.2-0.5$ \\
\hline
\end{tabular}

Figure 2. Cont. 
miR-499a-5p

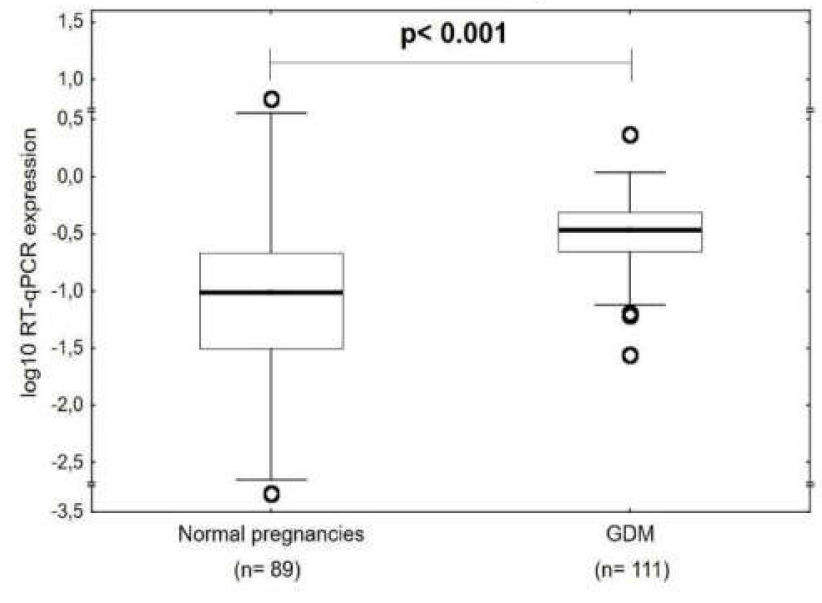

miR-499a-5p

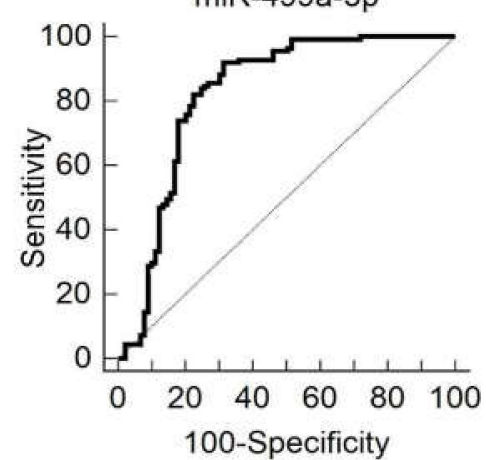

\begin{tabular}{|l|r|}
\hline Area under the ROC curve (AUC) & 0.824 \\
\hline
\end{tabular}

\begin{tabular}{l|r} 
Standard Error & 0.0333
\end{tabular}

95\% Confidence interval 0.764 to 0.874

Significance level P (Area $=0.5$

Estimated sensitivity at fixed specificity \begin{tabular}{l|l|l|l|}
\hline Specificity & Sensitivity & $95 \% \mathrm{Cl}$ & Criterion
\end{tabular} \begin{tabular}{|l|c|c|c|}
\hline 90.00 & 28.83 & 3.60 to 63.62 & $>0.462757253$ \\
\hline
\end{tabular}

\begin{tabular}{|l|r|r|r|r|r|r|r|r|}
\hline Criterion & Sensitivity & $95 \% \mathrm{Cl}$ & Specificity & $95 \% \mathrm{Cl}$ & +LR & $95 \% \mathrm{Cl}$ & -LR & $95 \% \mathrm{Cl}$ \\
\hline$>0.12646684$ & 91.89 & $85.2-96.2$ & 68.54 & $57.8-78.0$ & 2.92 & $2.1-4.0$ & 0.12 & $0.06-0.2$ \\
\hline
\end{tabular}

Z

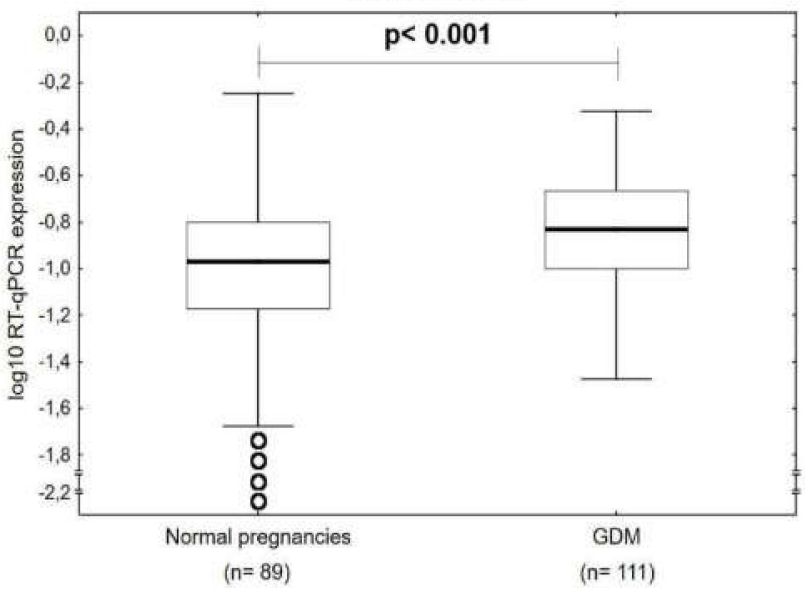

miR-574-3p

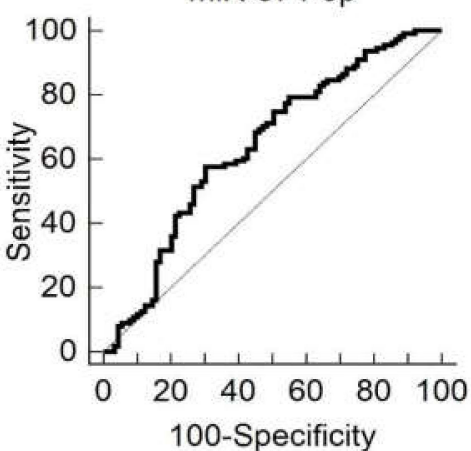

00-Specificity

\begin{tabular}{|l|r|}
\hline Area under the ROC curve (AUC) & 0.640 \\
\hline Standard Error & 0.0401 \\
\hline $95 \%$ Confidence interval & 0.570 to 0.707 \\
\hline Significance level P (Area $=0.5$ ) & 0.0005 \\
\hline
\end{tabular}

Estimated sensitivity at fixed specificity \begin{tabular}{l|l|l|l} 
Specificity & Sensitivity & $95 \% \mathrm{Cl}$ & Criterion \\
\hline
\end{tabular} \begin{tabular}{|l|c|c|c|}
\hline 90.00 & 10.81 & 2.70 to 28.83 & $>0.275544234$ \\
\hline
\end{tabular}

\begin{tabular}{|l|r|r|r|r|r|r|r|r|}
\hline Criterion & Sensitivity & $95 \% \mathrm{Cl}$ & Specificity & $95 \% \mathrm{Cl}$ & $+\mathrm{LR}$ & $95 \% \mathrm{Cl}$ & $-\mathrm{LR}$ & $95 \% \mathrm{Cl}$ \\
\hline$>0.132225234$ & 57.66 & $47.9-67.0$ & 69.66 & $59.0-79.0$ & 1.90 & $1.3-2.7$ & 0.61 & $0.5-0.8$ \\
\hline
\end{tabular}

Figure 2. Postpartal microRNA expression profile in mothers with a history of GDM pregnancies. (A-Z) Upregulation of miR-1-3p, miR-16-5p, miR-17-5p, miR-20a-5p, miR-20b-5p, miR-21-5p, miR-23a-3p, miR-24-3p, miR-26a-5p, miR-29a-3p, miR-100-5p, miR-103a-3p, miR-125b-5p, miR-126-3p, miR-130b-3p, miR-133a-3p, miR-143-3p, miR-145-5p, miR-146a-5p, miR-181a-5p, miR-195-5p, miR-199a-5p, miR-221-3p, miR-342-3p, miR-499a-5p, and miR-574-3p was observed in mothers after GDM pregnancies when the comparison to the controls was performed using Mann-Whitney test. Receivers operating characteristic (ROC) curves were constructed to calculate the area under the curve (AUC), the best cut-off point (criterion), the sensitivity, specificity, likelihood ratio positive (LR+), and likelihood ratio negative (LR-) for particular microRNA. In addition, respective sensitivity at 90.0\% 
specificity was reported for miR-1-3p (43.24\%), miR-16-5p (20.72\%), miR-17-5p (21.62\%), miR-20a-5p (10.81\%), miR-20b-5p (38.74\%), miR-21-5p (28.83\%), miR-23a-3p (17.12\%), miR-24-3p (0.90\%), miR-26a-5p (20.72\%), miR-29a-3p (21.62\%), miR-100-5p (13.51\%), miR-103a-3p (30.63\%), miR-125b-5p (9.91\%), miR-126-3p (9.91\%), miR-130b-3p (3.60\%), miR-133a-3p (18.92\%), miR-143-3p (13.51\%), miR-145-5p (7.21\%), miR-146a-5p (17.12\%), miR-181a-5p (31.53\%), miR-195-5p (16.22\%), miR-199a-5p (15.32\%), miR-221-3p (31.53\%), miR-342-3p (1.8\%), miR-499a-5p (28.83\%), and miR-574-3p (10.81\%).

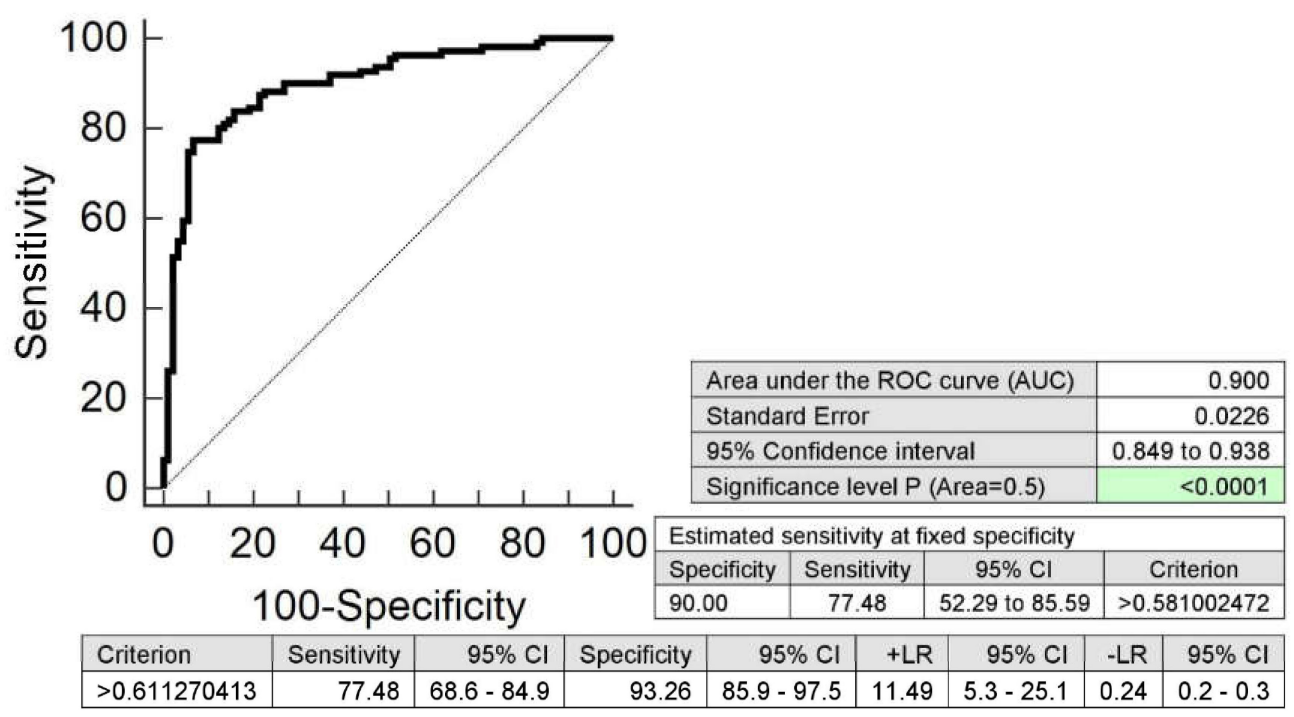

Figure 3. Combined postpartal screening of microRNAs in the identification of mothers with a history of GDM at an increased cardiovascular risk. Postpartal combined screening of miR-1-3p, miR-16-5p, miR-17-5p, miR-20b-5p, miR-21-5p, miR-23a-3p, miR-26a-5p, miR-29a-3p, miR-103a-3p, miR-133a-3p, miR-146a-5p, miR-181a-5p, miR-195-5p, miR-199a-5p, miR-221-3p, and miR-499a-5p showed the highest accuracy for the identification of mothers with a prior exposure to GDM at a higher risk of later development of diabetes mellitus, and cardiovascular/cerebrovascular diseases.

2.2. Expression Profile of MicroRNAs Associated with Diabetes Mellitus and Cardiovascular/Cerebrovascular Diseases in Mothers after GDM Pregnancies with Regard to the Treatment Strategies (Diet Only and/or Diet and Therapy)

Concurrently, it was observed that the expression of miR-1-3p $(p<0.001, p=0.013), \operatorname{miR}-16-5 p$ $(p<0.001, p=0.057)$, miR-17-5p $(p<0.001, p=0.035), \operatorname{miR}-20 \mathrm{~b}-5 \mathrm{p}(p<0.001, p=0.002), \mathrm{miR}-21-5 \mathrm{p}$ $(p<0.001, p<0.001)$, miR-23a-3p $(p<0.001, p=0.096), \operatorname{miR}-26 a-5 p(p<0.001, p=0.002)$, miR-29a-3p $(p<0.001, p<0.001)$, miR-103a-3p $(p<0.001, p=0.001), \operatorname{miR}-146 a-5 p(p<0.001, p=0.019)$, miR-181a-5p $(p<0.001, p=0.003)$, miR-195-5p $(p<0.001, p<0.001), \operatorname{miR}-199 \mathrm{a}-5 \mathrm{p}(p<0.001, p=0.002), \mathrm{miR}-221-3 \mathrm{p}$ $(p<0.001, p<0.001)$, and miR-499a-5p $(p<0.001, p<0.001)$ differed significantly or showed a trend toward statistical significance between the groups of mothers affected with GDM regardless of the treatment strategies (diet only and/or the combination of diet and therapy) and the controls (Figure 4). Nevertheless, miR-133a-3p was upregulated just in the group of patients previously affected with GDM on diet only ( $p=0.001$ ) (Figure 4 ).

Due to a low number of women on the diet and therapy $(n=18)$ in our studied group of patients previously affected with GDM, we decided not to perform ROC curve analysis for particular groups of patients regarding the GDM treatment strategies (diet only and/or combination of diet and therapy).

No difference in microRNA expression profiles was observed between the groups of mothers with a history of GDM on diet only and on the combination of diet and therapy ( $p=1.0$ for nearly all examined microRNAs). 
A

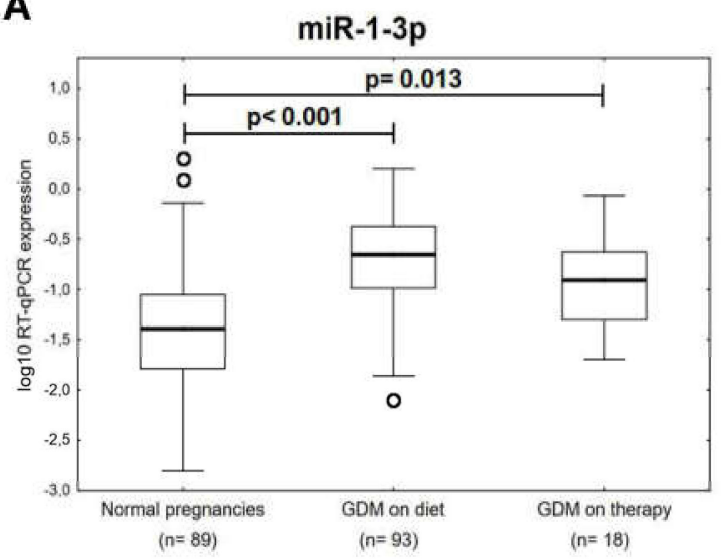

C

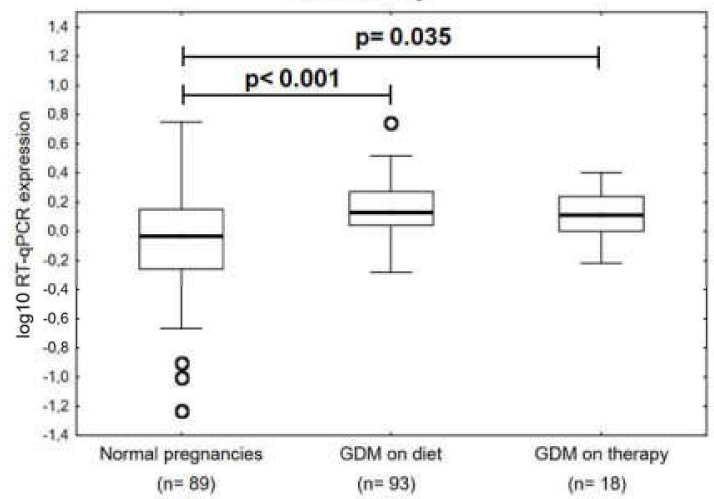

E

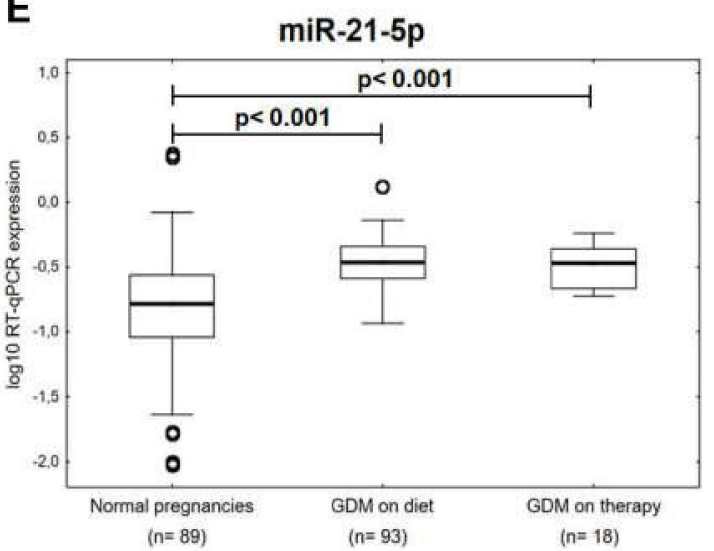

B

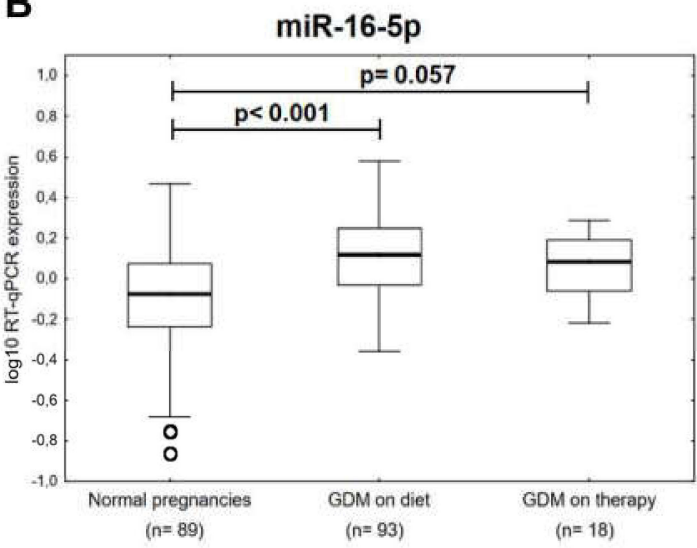

D

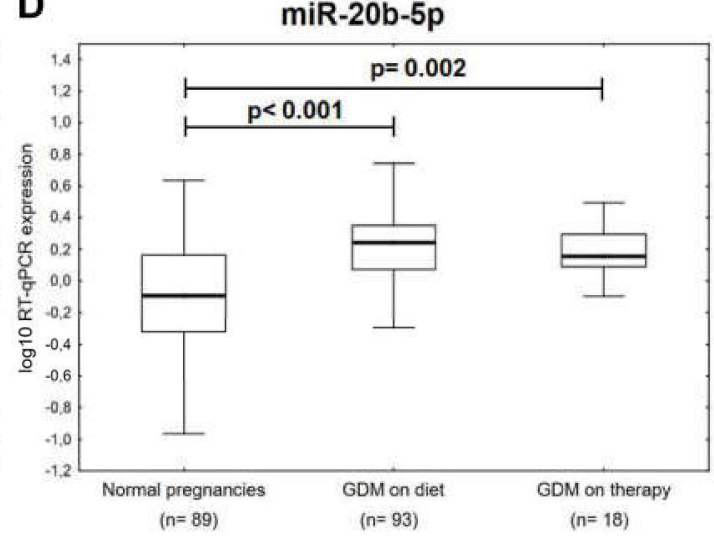

$\mathbf{F}$

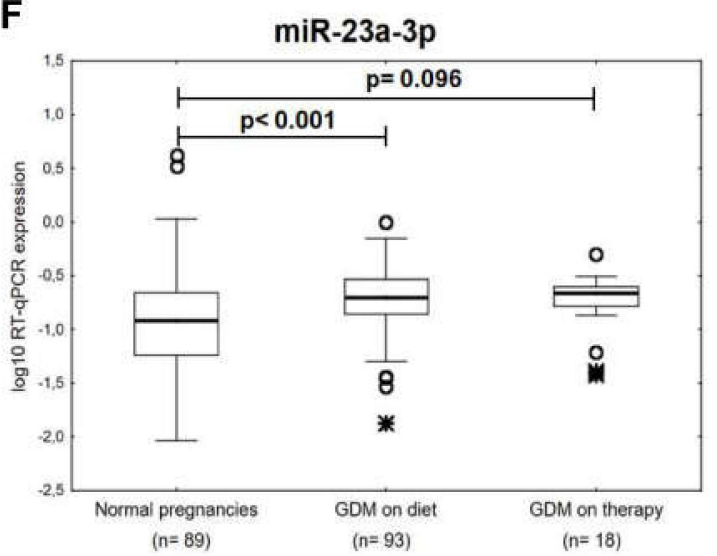

Figure 4. Cont. 
G
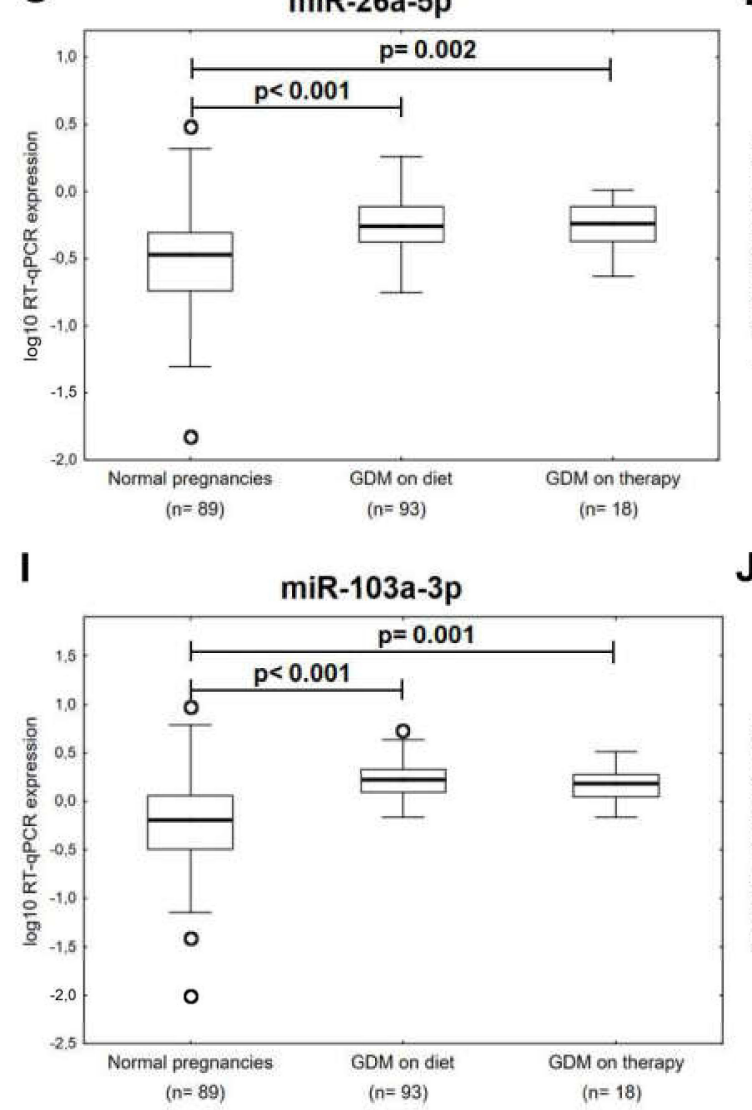

K

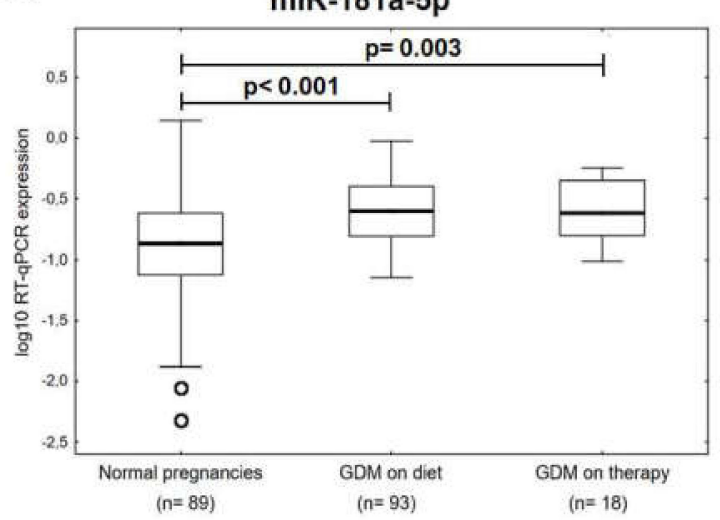

H

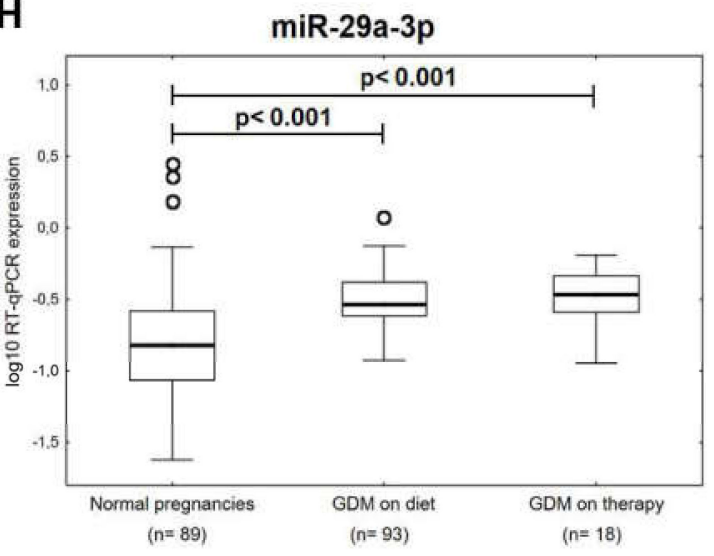

J

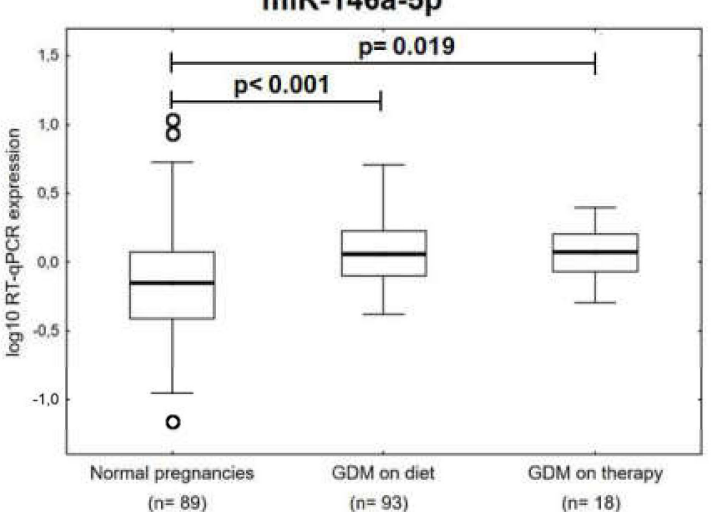

$\mathbf{L}$

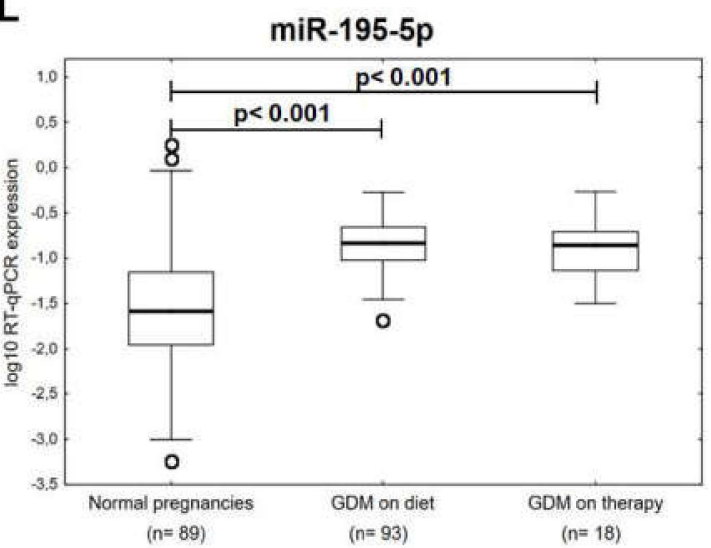

Figure 4. Cont. 
M

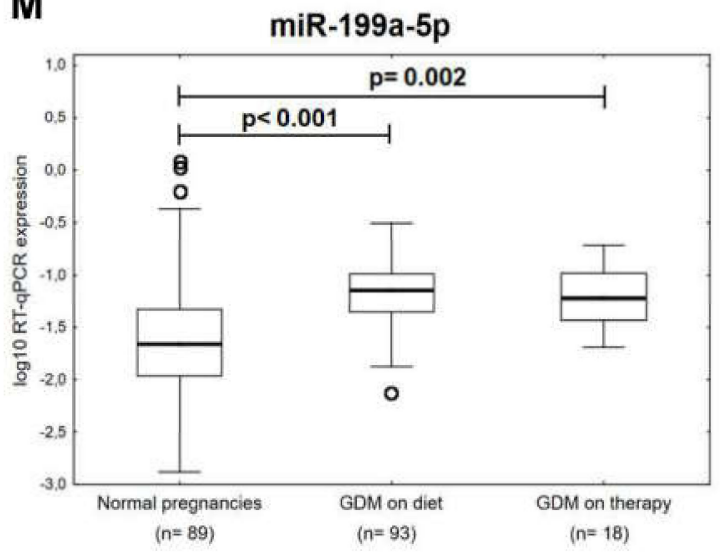

0

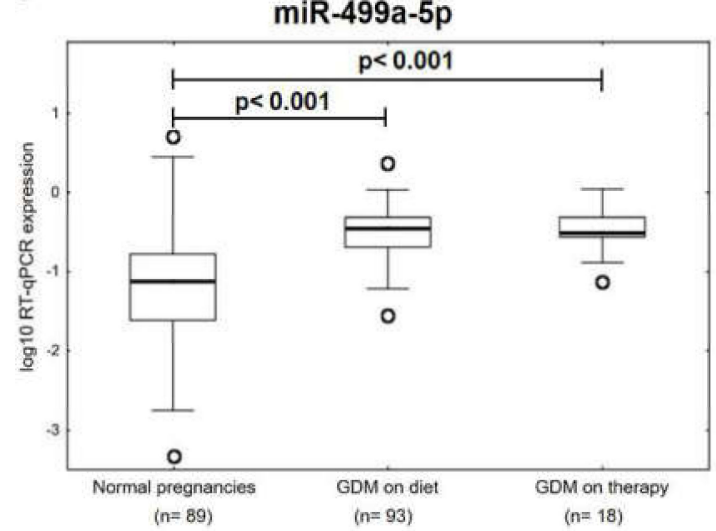

N

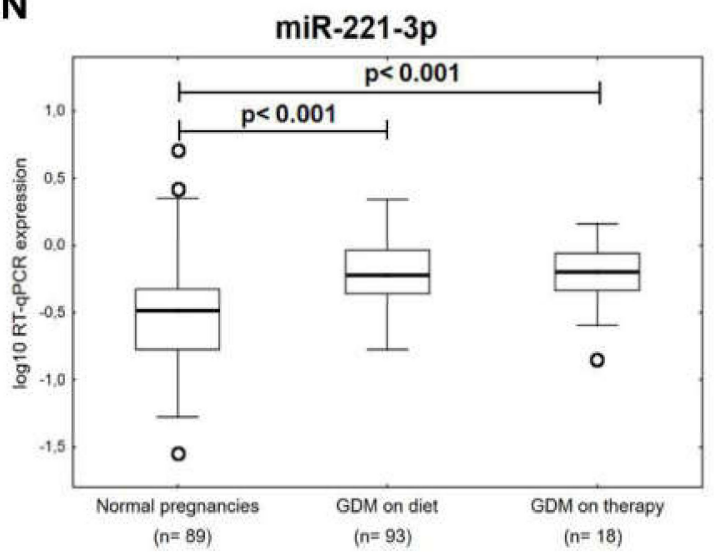

$\mathbf{P}$

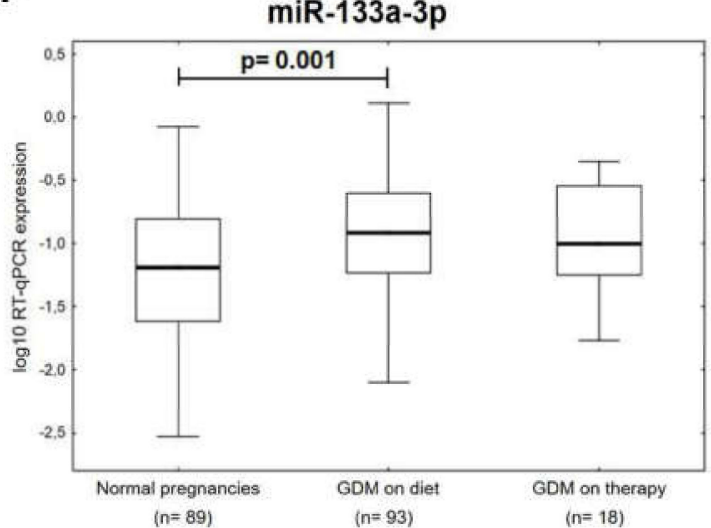

Figure 4. Postpartal microRNA expression profile in mothers with a history of GDM pregnancies with regard to the treatment strategies. (A-O) Upregulation or trend towards upregulation of miR-1-3p, miR-16-5p, miR-17-5p, miR-20b-5p, miR-21-5p, miR-23a-3p, miR-26a-5p, miR-29a-3p, miR-103a-3p, miR-146a-5p, miR-181a-5p, miR-195-5p, miR-199a-5p, miR-221-3p, and miR-499a-5p was observed in mothers after GDM pregnancies regardless of the treatment strategies (diet only and/or the combination of diet and therapy). (P) Upregulation of miR-133a-3p was observed in mothers after GDM pregnancies on diet only.

\subsection{Information on MicroRNA-Gene-Biological Pathway Interactions}

The extensive file of predicted targets of all microRNAs aberrantly expressed in whole peripheral blood of mothers with a history of GDM indicates that a large group of genes is involved in biological pathways related to insulin signaling, type 1 diabetes mellitus, and type 2 diabetes mellitus (Tables $2-4$ ).

Table 2. A list of predicted targets of appropriate microRNAs dysregulated in whole peripheral blood of patients with a history of GDM in relation to insulin signaling pathway using miRWalk2.0 database.

\begin{tabular}{cc}
\hline microRNA & Predicted Targets \\
\hline miR-1 & CALM2, CBL, IKBKB, KRAS, PHKG2, PIK3R5, PTPN1, PTPRF, TRIP10 \\
\hline miR-16-5p & IKBKB, PHKA1, PRKAR1A, MAP2K1, RAF1, IRS4, MKNK1, EXOC7, FASN \\
\hline miR-17-5p & PHKA2, CRK, GRB2, PDE3A, PHKG1, PIK3R2, PRKAA2, PRKAR2A, MAPK9, PRKX, \\
& PPP1R3B, HK1, PCK1, SREBF1 \\
\hline miR-20a-5p & BRAF, MKNK2, CRK, SLC2A4, TRIP10, KRAS, PCK1 \\
\hline miR-20b-5p & CRK, GRB2, PDE3A, PHKA2, PHKG1, PIK3R2, PRKAA2, PRKAR2A, MAPK9, PRKX, \\
\hline miR-21-5p & PPP1R3B, HK1, PCK1, SREBF1 \\
\hline
\end{tabular}


Table 2. Cont.

\begin{tabular}{cc}
\hline microRNA & Predicted Targets \\
\hline miR-23a-3p & G6PC, IRS2, IKBKB, PIK3CB, FASN, PRKAG3 \\
\hline miR-24-3p & IKBKB, PIK3CB, PTPRF, SHC2, INPP5K, PHKG1, PRKAG3 \\
\hline miR-26a-5p & G6PC, MKNK2, GYS2, PPP1R3D, RHOQ, KRAS, PRKAG1, PYGL \\
\hline miR-29a-3p & NRAS, EIF4E2, CALM3 \\
\hline miR-100-5p & MTOR \\
\hline miR-103a-3p & FAS, RAPGEF1, PDE3B, ACACB, PHKAR1A, PRKCI, IRS2, LIPE, PRKC2, MAPK3, \\
\hline miR-125b-5p & TRIP10, CBLC, CALML5 \\
\hline miR-126-3p & PHKA1, RAF1, ACACB, FLOT2, HK2, EIF4E2, PHKG1 \\
\hline miR-130b-3p & RPS6KB1, MAP2K1, SOS2, FLOT2, EXOC7, PHKG2, PIK3CA, PRKC2, TSC2, PRKAG3 \\
\hline miR-133a-3p & PRKAB1 \\
\hline miR-143-3p & FOXO1, KRAS, HK2, PHKG2, MAPK3, MAPK9, SREBF1 \\
\hline miR-145-5p & IRS1, IRS2, PIK3R5, PRKAG3 \\
\hline miR-181a-5p & NRAS, AKT3, SOCS4, HK2, PDE3B, PPP1R3C, PRKAR2A, MAPK1, PPP1R3D, PRKAA1 \\
\hline miR-195-5p & IKBKB, PHKA1, PRKAR1A, MAP2K1, RAF1, IRS4, MKNK1, EXOC7, FASN \\
\hline miR-199a-5p & PRKX, PCK1, IRS1, SLC2A4, MAPK9, RHEB, PRKAR1A \\
\hline miR-221-3p & AKT3, PIK3CD, MAPK10 \\
\hline miR-342-3p & PDPK1, INSR, PHKG2, EIF4E2, PIK3CD, RPS6KB2 \\
\hline miR-499a-5p & AKT2, CRK, KRAS, PIK3CD, PRKAR1A, SOS2 \\
\hline miR-574-3p & PRKCZ, HK1 \\
\hline &
\end{tabular}

Table 3. A list of predicted targets of appropriate microRNAs dysregulated in whole peripheral blood of patients with a history of GDM in relation to type 1 diabetes mellitus pathway using miRWalk2.0 database.

\begin{tabular}{|c|c|}
\hline microRNA & Predicted Targets \\
\hline miR-1 & CD28, LTA \\
\hline miR-16-5p & HLA-DQA1 \\
\hline miR-17-5p & FASLG, CD28, HLA-DQA, GAD2, HLA-DPA1 \\
\hline miR-20a-5p & IL12A \\
\hline miR-20b-5p & HLA-DOA, FASLG, CD28, HLA-DPA1, GAD2 \\
\hline miR-21-5p & HLA-DPB1, FASLG, IL12A \\
\hline miR-23a-3p & IFNG \\
\hline $\operatorname{miR}-24-3 p$ & CD28, CD86, IFNG, FASLG, IL1B, HLA-DOA \\
\hline miR-26a-5p & HLA-DPB1, HLA-DPA1, HLA-A, IFNG \\
\hline miR-29a-3p & HLA-DQA2 \\
\hline miR-103a-3p & HLA-DPB1, CD80 \\
\hline miR-125b-5p & PRF-1 \\
\hline miR-130b-3p & HLA-DOA, HLA-DQB1, HLA-A, HLA-B, HLA-C, HLA-G \\
\hline miR-133a-3p & HLA-DOA, CD28, GAD2, LTA \\
\hline miR-143-3p & HLA-DOA, HLA-DPB1, HLA-DPA1, IFNG, CD28 \\
\hline miR-146a-5p & CD80, CD86, PRF1, ICA1, HLA-C, GAD2 \\
\hline miR-181a-5p & IL2, HLA-E, IL1A \\
\hline miR-195-5p & HLA-DQA1 \\
\hline miR-199a-5p & G2MB, ICA1,TNF \\
\hline miR-221-3p & HLA-DQA1, PTPRN \\
\hline miR-342-3p & PTPRN2, HLA-A, HLA-F \\
\hline
\end{tabular}


Table 4. A list of predicted targets of appropriate microRNAs dysregulated in whole peripheral blood of patients with a history of GDM in relation to type 2 diabetes mellitus pathway using miRWalk2.0 database.

\begin{tabular}{|c|c|}
\hline microRNA & Predicted Targets \\
\hline miR-16-5p & CACNA1E, IKBKB, IRS4 \\
\hline $\operatorname{miR}-17-5 p$ & PIK3R2, MAPK9, HK1 \\
\hline miR-20a-5p & SLC2A4 \\
\hline $\operatorname{miR}-20 b-5 p$ & MAPK9, HK1 \\
\hline $\operatorname{miR}-23 a-3 p$ & IRS2, IKBKB, PIK3CB \\
\hline miR-24-3p & IKBKB, PIK3CB, KCNJ11 \\
\hline $\operatorname{miR}-26 a-5 p$ & PRKCD \\
\hline miR-29a-3p & CACNA1A, CACNA1B \\
\hline miR-100-5p & MTOR \\
\hline miR-103a-3p & CACNA1E, IRS2, PRKCZ, MAPK3 \\
\hline $\operatorname{miR}-125 b-5 p$ & HK2 \\
\hline $\operatorname{miR}-130 b-3 p$ & PIK3CA, PRKCZ \\
\hline miR-143-3p & CACNA1A, HK2, PRKCE, MAPK3, MAPK9 \\
\hline $\operatorname{miR}-145-5 p$ & IRS2, IRS1, PIK3R5 \\
\hline miR-146a-5p & PRKCE \\
\hline miR-181a-5p & SOCS4, HK2, MAPK1 \\
\hline $\operatorname{miR}-195-5 p$ & CACNA1E, IKBKB, IRS4 \\
\hline miR-199a-5p & IRS1, SLC2A4, MAPK9, PKM, TNF, CACNA1G \\
\hline $\operatorname{miR}-221-3 p$ & PIK3CD, MAPK10 \\
\hline $\operatorname{miR}-342-3 p$ & CACNA1C, INSR, PIK3CD \\
\hline miR-499a-5p & PRKCE, PIK3CD \\
\hline miR-574-3p & PRKCZ, HK1 \\
\hline
\end{tabular}

The miRWalk database and the predicted target module were used to provide information on predicted interactions between appropriate microRNAs and specific genes involved in human biological pathways such as insulin signaling, type 1 diabetes mellitus, and type 2 diabetes mellitus.

\section{Discussion}

Postpartal expression profile of cardiovascular/cerebrovascular disease associated microRNAs was assessed 3-11 years after the delivery in whole peripheral blood of young and middle-aged mothers with a prior exposure to GDM with the aim to identify a high-risk group of mothers at risk of later development of diabetes mellitus and cardiovascular and cerebrovascular diseases who would benefit from implementation of early primary prevention strategies and long-term follow-up. The hypothesis of the assessment of cardiovascular risk in women was based on the knowledge that a serious of microRNAs play a role in the pathogenesis of diabetes mellitus and cardiovascular/cerebrovascular diseases (Table 1) [11-170].

As expected, the expression profile of microRNAs differed between mothers affected with GDM and controls. Abnormal expression profile of multiple microRNAs was found in mothers with a history of GDM (26/29 studied microRNAs: miR-1-3p, miR-16-5p, miR-17-5p, miR-20a-5p, miR-20b-5p, miR-21-5p, miR-23a-3p, miR-24-3p, miR-26a-5p, miR-29a-3p, miR-100-5p, miR-103a-3p, miR-125b-5p, miR-126-3p, miR-130b-3p, miR-133a-3p, miR-143-3p, miR-145-5p, miR-146a-5p, miR-181a-5p, miR-195-5p, miR-199a-5p, miR-221-3p, miR-342-3p, miR-499a-5p, and miR-574-3p). 
Nevertheless, when the ROC curve analysis was performed, only 16/26 microRNAs (miR-1-3p, miR-16-5p, miR-17-5p, miR-20b-5p, miR-21-5p, miR-23a-3p, miR-26a-5p, miR-29a-3p, miR-103a-3p, miR-133a-3p, miR-146a-5p, miR-181a-5p, miR-195-5p, miR-199a-5p, miR-221-3p, and miR-499a-5p) with aberrant postpartal expression profile in whole peripheral blood of mothers with a prior exposure to GDM showed a higher sensitivity, ranging from $15.32 \%$ to $43.24 \%$, at $10.0 \%$ FPR.

Screening based on the combination of these particular microRNAs was superior over using individual microRNAs, since it showed the highest accuracy for mothers with a history of GDM (AUC $0.900, p<0.001$, sensitivity $77.48 \%$, specificity $93.26 \%$, cut off $>0.611270413$ ). It was able to identify $77.48 \%$ mothers with an increased cardiovascular risk at $10.0 \%$ FPR.

Subsequently, epigenetic profile was compared between groups with regard to the treatment strategy (GDM on diet only, GDM on the combination of diet and therapy). The upregulation or trend towards upregulation of almost all studied microRNAs (with the exception of miR-133a-3p) was present in both groups of mothers affected with GDM regardless of the treatment strategies.

Previously, we demonstrated that pregnancy-related complications, such as gestational hypertension, preeclampsia, and/or fetal growth restriction, were associated with postpartum alterations in gene expression of cardiovascular/cerebrovascular disease-associated microRNAs [1-3].

Likewise, a previous occurrence of $\mathrm{GH}$, as well as a previous occurrence of GDM, was associated with the upregulation of miR-20a-5p, miR-143-3p, miR-146a-5p, miR-181a-5p, miR-199a-5p, miR-221-3p, and miR-499a-5p [1-3].

Moreover, the compilation of data resulting from our previous and current studies showed that the upregulation of miR-17-5p, miR-20b-5p, miR-29a-3p, and miR-126-3p was a mutual phenomenon observed in women with a prior exposure to GH, severe PE, and/or GDM [1-3].

In addition, a history of GH, early PE, and/or GDM was associated with postpartal upregulation of miR-1-3p and miR-17-5p. Parallel, women affected with GH and/or late PE showed similar postpartal expression profile (upregulation of miR-17-5p, miR-20b-5p, and miR-29a-3p) as women with a history of GDM [1-3].

Alike, as in women affected with severe PE and/or early PE, upregulation of miR-133a-3p was also present in women affected with GDM. Moreover, a history of severe PE and/or GDM was associated with upregulation of miR-130b-3p [1-3].

Furthermore, women with a history of GDM demonstrated upregulation of miR-100-5p, miR-125b-5p, miR-133a-3p, and miR-145-5p as women with prior exposure to PE and/or FGR with abnormal Doppler parameters [1-3].

On the other hand, our study revealed that upregulation of miR-16-5p, miR-21-5p, miR-23a-3p, miR-24-3p, miR-26a-5p, miR-103a-3p, miR-195-5p, miR-342-3p, and miR-574-3p represented a unique feature of aberrant expression profile of women with a prior exposure to GDM.

Interestingly, interaction network analysis via Cytoscale V.3.6.1 revealed that miR-145-5p together with miR-875-5p are upregulated microRNAs that target the most genes in gestational diabetes mellitus [125].

In addition, increased expression of miR-126-3p and miR-130b-3p was observed in human umbilical vein endothelial cells (HUVECs) derived from GDM patients [98].

Moreover, a set of circulating microRNAs associated with cardiovascular/cerebrovascular diseases (miR-16-5p, miR-17-5p, miR-20a-5p, miR-29a-3p, miR-125b-5p, and miR-195-5p) was reported by several independent studies $[20,21,80,92,143,172,173]$ to be upregulated during various gestational ages in serum or plasma of mothers with already-diagnosed GDM or mothers who were destined to develop GDM later during pregnancy. The data resulting from these prenatally ongoing studies and interaction network analysis $[20,21,80,92,98,125,143,172,173]$ may support our current finding referring to upregulated expression profile of these particular microRNAs in whole peripheral blood of women with a prior exposure to GDM.

In addition, from the portfolio of microRNAs we tested, just miR-16-5p, miR-17-5p, miR-20a-5p, and miR-29a were identified by other investigators as the most promising biomarkers of GDM or the 
best predictors of GDM, respectively $[21,173]$, which is consistent with our current finding, since during the course of postpartum screening, miR-16-5p (20.72\%), miR-17-5p (21.62\%), and miR-29a-3p $(21.62 \%)$ upregulation persisted in a larger proportion of women with a prior exposure to GDM.

In compliance with our study, which showed a low sensitivity of miR-20a-5p (10.81\%) to select women with a higher cardiovascular risk after GDM gestation based on its upregulated profile, miR-20a-5p also added little value as the predictor of GDM due to its low predictive value based on its upregulated profile (ROC, AUC just 0.740) [21,173].

This study demonstrated that the dysregulation of at least four microRNAs (miR-16-5p, miR-17-5p, miR-29a-3p, and miR-195-5p) induced by GDM-complicated pregnancy in maternal circulation (plasma or serum) is present as well in circulation (whole peripheral blood) of a larger proportion of mothers $(20.72 \%$, $21.62 \%, 21.62 \%, 16.22 \%$ ) with hindsight ( 3 to 11 years after the delivery) after the exposure to GDM.

It is obvious that changes in epigenome induced by GDM, which are persistently present in maternal circulation, may cause later development of diabetes mellitus and cardiovascular and cerebrovascular diseases in women with a prior exposure to GDM.

However, most of microRNAs, which were the subjects of our interest, have not yet been observed by other investigators to be dysregulated in maternal circulation (peripheral blood, plasma, or serum samples) of women with clinically established GDM or before the onset of GDM [20,21,37,80,92,143,149,172-176]. So, it is feasible that epigenetic profiles of a series of microRNAs have also been changing with time by force of various circumstances as a result of the interaction between genetic and environmental factors [177].

\section{Materials and Methods}

\subsection{Participants}

The study included a prospectively collected cohort of Caucasian mothers with a history of GDM $(n=111)$ and age-matched mothers after normal course of gestation $(n=89)$. In-person visit was conducted 3-11 years after the pregnancy ended. Of the 111 GDM pregnancies, 93 were on diet only and 18 were on the combination of diet and therapy (17 patients required insulin administration and in 1 patient metformin, an oral hypoglycemic agent, was prescribed).

The clinical characteristics of mothers after GDM-complicated pregnancies are presented in Tables 5-7.

Gestational diabetes mellitus is defined as any degree of glucose intolerance with onset or first recognition during pregnancy $[10,178,179]$. The International Association of Diabetes and Pregnancy Study Groups' (IADPSG) recommendations on the diagnosis and classification of hyperglycemia in pregnancy were followed [10]. The first screening phase detects, during the first trimester of gestation, women with overt diabetes (fasting plasma glucose level is $\geq 7.0 \mathrm{mmol} / \mathrm{L}$ ) and women with GDM (fasting plasma glucose level $\geq 5.1 \mathrm{mmol} / \mathrm{L}<7.0 \mathrm{mmol} / \mathrm{L}$ ). The second screening phase, 2-h 75-g OGTT, at 24-28 weeks of gestation is done in all women not previously found to have overt diabetes or GDM, and identifies GDM if fasting plasma glucose level is $\geq 5.1 \mathrm{mmol} / \mathrm{L}$ or 1-h plasma glucose is $\geq 10.0 \mathrm{mmol} / \mathrm{L}$ or 2 -h plasma glucose is $\geq 8.5 \mathrm{mmol} / \mathrm{L}$ [10].

Patients demonstrating other pregnancy-related complications, such as gestational hypertension, preeclampsia, fetal growth restriction, premature rupture of membranes, in utero infections, fetal anomalies or chromosomal abnormalities, and fetal demise in utero or stillbirth, were not involved in the study.

Written informed consent was provided for all participants included in the study. The study was approved by the Ethics Committee of the Institute for the Care of the Mother and Child, Prague, Czech Republic (grant no. AZV 16-27761A, long-term monitoring of complex cardiovascular profile in the mother, fetus, and offspring descending from pregnancy-related complications, date of approval: 28 May 2015) and by the Ethics Committee of the Third Faculty of Medicine, Prague, Czech Republic (grant no. AZV 16-27761A, long-term monitoring of complex cardiovascular profile in the mother, fetus, and offspring descending from pregnancy-related complications, date of approval: 27 March 2014). 
Table 5. Characteristics of cases and controls, pre-existing cardiovascular risk factors before gestation.

\begin{tabular}{|c|c|c|c|}
\hline & $\begin{array}{l}\text { Normotensive Term Pregnancies } \\
\qquad(n=89)\end{array}$ & $\begin{array}{l}\text { GDM on Diet Only } \\
\quad(n=93)\end{array}$ & $\begin{array}{l}\text { GDM on Diet and Therapy } \\
\qquad(n=18)\end{array}$ \\
\hline Rheumatoid arthritis & $0(0 \%)$ & $1(1.08 \%)$ & $0(0 \%)$ \\
\hline SLE & $0(0 \%)$ & $0(0 \%)$ & $0(0 \%)$ \\
\hline On blood pressure treatment & $0(0 \%)$ & $0(0 \%)$ & $0(0 \%)$ \\
\hline Hypercholesterolemia & $0(0 \%)$ & $1(1.08 \%)$ & $0(0 \%)$ \\
\hline $\begin{array}{l}\text { Dispensarisation at Dpt. of Cardiology } \\
\text { (valve problems and heart defects) }\end{array}$ & $0(0 \%)$ & $\begin{array}{c}4(4.30 \%) \\
\text { Mitral valve prolapse } \\
\text { Heart arrhythmia } \\
\text { Hypertrophic cardiomyopathy } \\
\text { AV nodal reentrant tachycardia }\end{array}$ & $\begin{array}{c}1(5.55 \%) \\
\text { Mitral valve regurgitation }\end{array}$ \\
\hline Chronic venous insufficiency & $0(0 \%)$ & $0(0 \%)$ & $0(0 \%)$ \\
\hline Thrombosis & $0(0 \%)$ & $2(2.15 \%)$ & $0(0 \%)$ \\
\hline $\begin{array}{l}\text { Presence of risk factors for chronic } \\
\text { kidney disease }\end{array}$ & $0(\%)$ & $\begin{array}{c}6(6.45 \%) \\
\text { Pyelonephritis } \\
\text { Glomerulonephritis } \\
\text { Ureteral stent } \\
\text { Nephrolithiasis } \\
\text { Hydronephrosis }\end{array}$ & $0(0 \%)$ \\
\hline Chronic kidney disease & $0(\%)$ & $\begin{array}{c}1(0.98 \%) \\
\text { Chronic renal insufficiency }\end{array}$ & $0(0 \%)$ \\
\hline
\end{tabular}


Table 6. Characteristics of cases and controls, data gained at follow-up.

\begin{tabular}{|c|c|c|c|c|c|}
\hline & $\begin{array}{l}\text { Normotensive Term Pregnancies } \\
\qquad(n=89)\end{array}$ & $\begin{array}{c}\text { GDM on Diet Only } \\
(n=93)\end{array}$ & $\begin{array}{l}\text { GDM on Diet and Therapy } \\
\qquad(n=18)\end{array}$ & $p$-value ${ }^{1}$ & $p$-value ${ }^{2}$ \\
\hline Age (years) & $38.33 \pm 0.38$ & $38.70 \pm 0.37$ & $38.61 \pm 0.80$ & 0.465 & 0.564 \\
\hline Time elapsed since delivery (years) & $5.75 \pm 0.20$ & $5.49 \pm 0.12$ & $5.33 \pm 0.20$ & 0.965 & 0.045 \\
\hline BMI & $23.15 \pm 0.38$ & $23.85 \pm 0.37$ & $27.21 \pm 0.83$ & 1.000 & 1.000 \\
\hline Normal $(<25)$ & $67(75.28 \%)$ & $70(75.27 \%)$ & $5(27.78 \%)$ & 0.633 & $<0.001$ \\
\hline Overweight $(\geq 25<30)$ & $18(20.22 \%)$ & $16(17.20 \%)$ & $9(50.00 \%)$ & & \\
\hline Obese $(\geq 30)$ & $4(4.49 \%)$ & $7(7.53 \%)$ & $4(22.22 \%)$ & & \\
\hline \multicolumn{4}{|c|}{ Smoking } & - & - \\
\hline Non-Smoker & $54(60.67 \%)$ & $78(83.87 \%)$ & $16(88.88 \%)$ & & \\
\hline Ex-smoker & $21(23.60 \%)$ & $6(6.45 \%)$ & $2(11.11 \%)$ & & \\
\hline Smoker & $14(15.73 \%)$ & $9(9.68 \%)$ & $0(0 \%)$ & & \\
\hline $\begin{array}{l}\text { Angina or heart attack in a first degree } \\
\text { relative before the age of } 60 \text { years }\end{array}$ & $2(2.22 \%)$ & $6(6.45 \%)$ & $2(11.11 \%)$ & - & - \\
\hline Atrial fibrillation & $0(0 \%)$ & $0(0 \%)$ & $0(0 \%)$ & - & - \\
\hline DM type I & $0(0 \%)$ & $0(0 \%)$ & $0(0 \%)$ & - & - \\
\hline DM type II & $0(0 \%)$ & $0(0 \%)$ & $0(0 \%)$ & - & - \\
\hline \multicolumn{4}{|c|}{ Fasting serum glucose levels } & - & - \\
\hline Normal (3.33-5.59 mmol/L) & $89(100.0 \%)$ & $90(96.77 \%)$ & $18(100.0 \%)$ & & \\
\hline High $(>5.59$ mmol/L) & $0(0 \%)$ & $3(3.23 \%)$ & $0(0 \%)$ & & \\
\hline \multicolumn{4}{|c|}{ Fasting serum total cholesterol levels } & - & - \\
\hline Normal (2.9-5.0 mmol/L) & $43(48.31 \%)$ & $51(54.84 \%)$ & $10(55.55 \%)$ & & \\
\hline High $(>5.0-7.9 \mathrm{mmol} / \mathrm{L})$ & $46(51.69 \%)$ & $41(44.09 \%)$ & $8(44.44 \%)$ & & \\
\hline Critical $(\geq 8.0 \mathrm{mmol} / \mathrm{L})$ & $0(0 \%)$ & $1(1.08 \%)$ & $0(0 \%)$ & & \\
\hline \multicolumn{4}{|c|}{ Fating serum LDL cholesterol levels } & 0.987 & 0.281 \\
\hline Normal (1.2-3.0 mmol/L) & $42(47.19 \%)$ & $44(47.31 \%)$ & $6(33.33 \%)$ & & \\
\hline $\operatorname{High}(>3.0 \mathrm{mmol} / \mathrm{L})$ & $47(52.81 \%)$ & $49(52.69 \%)$ & $12(66.67 \%)$ & & \\
\hline
\end{tabular}


Table 6. Cont.

\begin{tabular}{|c|c|c|c|c|c|}
\hline & $\begin{array}{l}\text { Normotensive Term Pregnancies } \\
\qquad(n=89)\end{array}$ & $\begin{array}{c}\text { GDM on Diet Only } \\
(n=93)\end{array}$ & $\begin{array}{l}\text { GDM on Diet and Therapy } \\
\qquad(n=18)\end{array}$ & $p$-value ${ }^{1}$ & $p$-value ${ }^{2}$ \\
\hline \multicolumn{4}{|c|}{ SBP } & - & - \\
\hline Normal (<140 mmHg) & $89(100.0 \%)$ & $92(98.92 \%)$ & $18(100.0 \%)$ & & \\
\hline High $(\geq 140-179 \mathrm{mmHg})$ & $0(0 \%)$ & $1(1.08 \%)$ & $0(0 \%)$ & & \\
\hline Critical ( $\geq 180 \mathrm{mmHg})$ & $0(0 \%)$ & $0(0 \%)$ & $0(0 \%)$ & & \\
\hline \multicolumn{4}{|c|}{ DBP } & - & - \\
\hline Normal $(<90 \mathrm{mmHg})$ & $88(98.88 \%)$ & $90(96.77 \%)$ & $17(94.44 \%)$ & & \\
\hline High ( $\geq 90-109 \mathrm{mmHg}$ ) & $1(1.12 \%)$ & $3(3.23 \%)$ & $1(5.56 \%)$ & & \\
\hline Critical ( $\geq 110 \mathrm{mmHg})$ & $0(0 \%)$ & $0(0 \%)$ & $0(0 \%)$ & & \\
\hline On blood pressure treatment & $0(0 \%)$ & $0(0 \%)$ & $0(0 \%)$ & - & - \\
\hline Chronic kidney disease & $0(\%)$ & $\begin{array}{c}1(0.98 \%) \\
\text { Chronic renal } \\
\text { insufficiency }\end{array}$ & $0(0 \%)$ & - & - \\
\hline Chronic venous insufficiency & $0(0 \%)$ & $0(0 \%)$ & $0(0 \%)$ & - & - \\
\hline Thrombosis & $0(0 \%)$ & $2(2.15 \%)$ & $0(0 \%)$ & - & - \\
\hline Relative QRISK ${ }^{\circledR} 3$-2018 risk score & $0.926 \pm 0.05$ & $0.889 \pm 0.04$ & $1.022 \pm 0.08$ & 1.0 & 1.0 \\
\hline \multicolumn{4}{|c|}{ Hormonal contraceptive use } & 0.040 & 0.474 \\
\hline No & $12(13.48 \%)$ & $5(5.38 \%)$ & $2(11.11 \%)$ & & \\
\hline In the past & $63(70.79 \%)$ & $80(86.02 \%)$ & $15(83.33 \%)$ & & \\
\hline Yes & $14(15.73 \%)$ & $8(8.60 \%)$ & $1(5.56 \%)$ & & \\
\hline \multicolumn{4}{|c|}{ Total number of pregnancies per patient } & 0.924 & 0.440 \\
\hline 1 & $9(10.11 \%)$ & $8(8.60 \%)$ & $2(11.11 \%)$ & & \\
\hline 2 & $44(49.44 \%)$ & $48(51.61 \%)$ & $6(33.33 \%)$ & & \\
\hline $3+$ & $36(40.45 \%)$ & $37(39.78 \%)$ & $10(55.55 \%)$ & & \\
\hline \multicolumn{4}{|c|}{ Total parity per patient } & 0.991 & 0.217 \\
\hline 1 & $13(14.61 \%)$ & $13(13.98 \%)$ & $2(11.11 \%)$ & & \\
\hline 2 & $62(69.66 \%)$ & $65(69.89 \%)$ & $10(55.55 \%)$ & & \\
\hline $3+$ & $14(15.73 \%)$ & $15(16.13 \%)$ & $6(33.33 \%)$ & & \\
\hline
\end{tabular}

Data are presented as mean \pm SE for continuous variables and as number (percent) for categorical variables. Statistically significant results are marked in bold. Continuous variables were compared using Mann-Whitney test. $p$-value ${ }^{1}$, the comparison among normal pregnancies and GDM on diet; $p$-value ${ }^{2}$, the comparison among normal pregnancies and GDM on diet and therapy, respectively. Categorical variables were compared using a chi-square test. GA, gestational age; SBP, systolic blood pressure; DBP, diastolic blood pressure; CS, caesarean section. 
Table 7. Characteristics of cases and controls, data gained during gestation

\begin{tabular}{|c|c|c|c|c|c|}
\hline & $\begin{array}{l}\text { Normotensive Term Pregnancies } \\
\qquad(n=89)\end{array}$ & $\begin{array}{l}\text { GDM on Diet Only } \\
(n=93)\end{array}$ & $\begin{array}{l}\text { GDM on Diet and Therapy } \\
\qquad(n=18)\end{array}$ & $p$-value ${ }^{1}$ & $p$-value ${ }^{2}$ \\
\hline $\begin{array}{l}\text { Maternal age at delivery } \\
\text { (years) }\end{array}$ & $32.62 \pm 0.36$ & $33.20 \pm 0.35$ & $33.28 \pm 0.83$ & 0.111 & 1.000 \\
\hline GA at delivery (weeks) & $39.90 \pm 0.10$ & $39.59 \pm 0.09$ & $39.20 \pm 0.23$ & 0.961 & 1.000 \\
\hline Fetal birth weight (g) & $3397.53 \pm 40.42$ & $3436.56 \pm 35.96$ & $3526.11 \pm 63.35$ & 1.000 & 1.000 \\
\hline \multicolumn{4}{|c|}{ Mode of delivery } & $<0.001$ & 0.015 \\
\hline Vaginal & $82(92.13 \%)$ & $57(61.29 \%)$ & $13(72.22 \%)$ & & \\
\hline CS & $7(7.87 \%)$ & $36(38.71 \%)$ & $5(27.78 \%)$ & & \\
\hline \multicolumn{4}{|c|}{ Fetal sex } & 0.476 & 0.281 \\
\hline Boy & $47(52.81 \%)$ & $54(58.06 \%)$ & $12(66.67 \%)$ & & \\
\hline Girl & $42(47.19 \%)$ & $39(41.94 \%)$ & $6(33.33 \%)$ & & \\
\hline \multicolumn{4}{|c|}{ Infertility treatment } & 0.006 & 0.846 \\
\hline Yes & $4(4.49 \%)$ & $16(17.20 \%)$ & $1(5.56 \%)$ & & \\
\hline No & $85(95.51 \%)$ & $77(82.80 \%)$ & $17(94.44 \%)$ & & \\
\hline
\end{tabular}

Data are presented as mean \pm SE for continuous variables and as number (percent) for categorical variables. Statistically significant results are marked in bold. Continuous variables were compared using Mann-Whitney test. $p$-value ${ }^{1}$, the comparison among normal pregnancies and GDM on diet; $p$-value ${ }^{2}$, the comparison among normal pregnancies and GDM on diet and therapy, respectively. Categorical variables were compared using a chi-square test. GA, gestational age; SBP, systolic blood pressure; DBP, diastolic blood pressure; CS, caesarean section. 


\subsection{Processing of Samples}

Homogenized cell lysates were prepared immediately after collection of whole peripheral blood samples (EDTA tubes, $200 \mu \mathrm{L}$ ) using QIAamp RNA Blood Mini Kit (Qiagen, Hilden, Germany, no: 52304).

Total RNA was extracted from homogenized cell lysates stored at $-80{ }^{\circ} \mathrm{C}$ using a mirVana microRNA Isolation kit (Ambion, Austin, USA, no: AM1560) and followed by an enrichment procedure for small RNAs. To minimize DNA contamination, the eluted RNA was treated for $30 \mathrm{~min}$ at $37^{\circ} \mathrm{C}$ with $5 \mu \mathrm{L}$ of DNase I (Thermo Fisher Scientific, CA, USA, no: EN0521). A RNA fraction highly enriched in short RNAs $(<200 \mathrm{nt})$ was obtained. The concentration and quality of RNA was assessed using a NanoDrop ND-1000 spectrophotometer (NanoDrop Technologies, USA). If the A(260/280) absorbance ratio of isolated RNA was 1.8-2.0 and the A(260/230) absorbance ratio was greater than 1.6, the RNA fraction was pure and used for the consecutive analysis.

\subsection{Reverse Transcriptase Reaction}

Individual microRNAs were reverse transcribed into complementary DNA (cDNA) in a total reaction volume of $10 \mu \mathrm{L}$ using microRNA-specific stem-loop RT primers, components of TaqMan MicroRNA Assays (Table 8), and TaqMan MicroRNA Reverse Transcription Kit (Applied Biosystems, Branchburg, USA, no: 4366597). Reverse transcriptase reactions were performed with the following thermal cycling parameters: $30 \mathrm{~min}$ at $16^{\circ} \mathrm{C}, 30 \mathrm{~min}$ at $42{ }^{\circ} \mathrm{C}, 5 \mathrm{~min}$ at $85^{\circ} \mathrm{C}$, and then held at $4{ }^{\circ} \mathrm{C}$ using a 7500 Real-Time PCR system (Applied Biosystems, Branchburg, USA).

Table 8. Characteristics of microRNAs involved in the study.

\begin{tabular}{|c|c|c|c|}
\hline Assay Name & miRBase ID & NCBI Location Chromosome & microRNA Sequence \\
\hline hsa-miR-1 & hsa-miR-1-3p & hr20: 61 & 5'-UGGAAUGUAAAGAAGUAUGUAU-3' \\
\hline hsa-miR-16 & hsa-miR-16-5p & Chr13: 50623109-50623197 [-] & 5'-UAGCAGCACGUAAAUAUUGGCG- 3' \\
\hline hsa-n & hsa-miR-17-5p & Chr13: 92002859-92002942 [+] & 5'-CAAAGUGCUUACAGUGCAGGUAG-3' \\
\hline hsa-miR-20a & hsa-miR-20a-5p & Chr13: 92003319-92003389 [+] & 5'-UAAAGUGCUUAUAGUGCAGGUAG-3' \\
\hline hsa-miR-20b & hsa-miR-20b-5p & ChrX: 133303839-133303907 [-] & 5'-CAAAGUGCUCAUAGUGCAGGUAG-3’ \\
\hline hsa-miR-21 & hsa-miR-21-5p & Chr17: 57918627-57918698 [+] & 5'-UAGCUUAUCAGACUGAUGUUGA-3' \\
\hline hsa-m & hsa-miR-23a-3p & Chr19: 13947401-13947473 [-] & 5'-AUCACAUUGCCAGGGAUUUCC-3’' \\
\hline hsa-miR-24 & hsa-miR-24-3p & $947101-13947173[-]$ & 5'-UGGCUCAGUUCAGCAGGAACAG-3' \\
\hline hsa-m & $26 a-5 p$ & Chr3: 38010895-38010971 [+] & 5'-UUCAAGUAAUCCAGGAUAGGCU-3' \\
\hline hsa-miR-29a & hsa-miR-29a-3p & Chr7: 130561506-130561569 [-] & 5'-UAGCACCAUCUGAAAUCGGUUA-3' \\
\hline hsa-miR-92a & hsa-miR-92a-3p & Chr13: 92003568-92003645 [+] & 5'-UAUUGCACUUGUCCCGGCCUGU-3' \\
\hline hsa-miR-100 & hsa-miR-100-5p & Chr11: 122022937-122023016 [-] & 5'-AACCCGUAGAUCCGAACUUGUG-3' \\
\hline hsa-miR-103 & hsa-miR-103a-3p & Chr20: 3898141-3898218 [+] & 5'-AGCAGCAUUGUACAGGGCUAUGA-3' \\
\hline hsa-miR-125b & hsa-miR-1 & Chr21: 17962557-17962645 [+] & 5’UCCCUGAGACCCUAACUUGUGA-3’ \\
\hline hsa-n & hsa-r & $65138[+]$ & 5'-UCGUACCGUGAGUAAUAAUGCG-3' \\
\hline hsa-miR-130b & hsa-mil & Chr22: 22007593-22007674 [+] & 5'-CAGUGCAAUGAUGAAAGGGCAU-3' \\
\hline hsa-m & hsa-m & Chr20: 61162119-61162220 [+] & 5'-UUUGGUCCCCUUCAACCAGCUG-3' \\
\hline hsa-m & hsa-n & $48808586[+]$ & 5'-UGAGAUGAAGCACUGUAGCUC-3’' \\
\hline hsa-miR-145 & $2-145-5 p$ & Chr5: 148810209-148810296 [+] & 5'-GUCCAGUUUUCCCAGGAAUCCCU-3' \\
\hline hsa-miR-146a & hsa-miR-146a-5p & Chr5: 159912359-159912457 [+] & 5'-UGAGAACUGAAUUCCAUGGGUU-3’ \\
\hline hsa-miR-155 & hsa-miR-155-5p & Chr21: 26946292-26946356 [+] & 5'-UUAAUGCUAAUCGUGAUAGGGGU-3’ \\
\hline hsa-miR-181a & hsa-miR-181a-5p & Chr9: 127454721-127454830 [+] & 5'-AACAUUCAACGCUGUCGGUGAGU-3' \\
\hline hsa-miR-195 & hsa-miR-195-5p & Chr17: 6920934-6921020 [-] & 5’UAGCAGCACAGAAAUAUUGGC-3’' \\
\hline hsa-miR-199a & hsa-miR-199a-5p & Chr19: 10928102-10928172 [-] & 5'-CCCAGUGUUCAGACUACCUGUUC-3' \\
\hline hsa-miR-210 & hsa-miR-210-3p & Chr11: 568089-568198 [-] & 5'-CUGUGCGUGUGACAGCGGCUGA-3’' \\
\hline hsa-miR-221 & hsa-miR-221-3p & ChrX: 45605585-45605694 [-] & 5'-AGCUACAUUGUCUGCUGGGUUUC-3’ \\
\hline hsa-miR-342-3p & hsa-miR-342-3p & Chr14: 100575992-100576090 [+] & 5'-UCUCACACAGAAAUCGCACCCGU-3' \\
\hline mmu-miR-499 & hsa-miR-499a-5p & Chr20: 33578179-33578300 [+] & 5’-UUAAGACUUGCAGUGAUGUUU-3’' \\
\hline hsa-miR-574-3p & hsa-miR-574-3p & Chr4: 38869653-38869748 [+] & 5'-CACGCUCAUGCACACACCCACA-3' \\
\hline
\end{tabular}

\subsection{Relative Quantification of MicroRNAs by Real-Time PCR}

The cDNA $(3 \mu \mathrm{L})$ was mixed with specific TaqMan MGB probes and primers (TaqMan MicroRNA Assay, Applied Biosystems, Branchburg, USA), and the components of the TaqMan Universal PCR 
Master Mix (Applied Biosystems, Branchburg, USA, no: 4318157). A total reaction volume was $15 \mu \mathrm{L}$. TaqMan PCR conditions were set up as described in the TaqMan guidelines for a 7500 Real-Time PCR system. All PCRs were performed in duplicates with the involvement of multiple negative controls such as NTC (water instead of cDNA sample), NAC (nontranscribed RNA samples), and genomic DNA (isolated from equal biological samples), which did not generate any signal during PCR reactions. The samples were considered positive if the amplification signal occurred at $\mathrm{Ct}<40$ (before the 40th threshold cycle).

The expression of particular microRNA was determined using the comparative Ct method [180] relative to normalization factor (geometric mean of two selected endogenous controls) [181]. Two noncoding small nucleolar RNAs (RNU58A and RNU38B) were optimal for qPCR data normalization in this setting. They demonstrated equal expression between children descending from normal and complicated pregnancies. RNU58A and RNU38B also served as positive controls for successful extraction of RNA from all samples and were used as internal controls for variations during the preparation of RNA, cDNA synthesis, and real-time PCR.

A reference sample, RNA fraction highly enriched for small RNAs isolated from the fetal part of one randomly selected placenta derived from gestation with normal course, was used throughout the study for relative quantification.

\subsection{Statistical Analysis}

Data normality was assessed using the Shapiro-Wilk test [182]. Since our experimental data did not follow a normal distribution, microRNA levels were compared between groups using the Kruskal-Wallis one-way analysis of variance with post hoc test for the comparison among multiple groups. The significance level was established at a $p$-value of $\mathrm{p}<0.05$.

Receivers operating characteristic (ROC) curves were constructed to calculate the area under the curve (AUC) and the best cut-off point for particular microRNA was used in order to calculate the respective sensitivity at $90.0 \%$ specificity (MedCalc Software bvba, Ostend, Belgium). For every possible threshold or cut-off value, the MedCalc program reports the sensitivity, specificity, likelihood ratio positive $(\mathrm{LR}+)$, likelihood ratio negative (LR-).

To select the optimal combinations of microRNA biomarkers, logistic regression was used (MedCalc Software bvba, Ostend, Belgium). The logistic regression procedure allowed us to analyses the relationship between one dichotomous dependent variable and one or more independent variables. Another method to evaluate the logistic regression model makes use of ROC curve analysis. In this analysis, the power of the model's predicted values to discriminate between positive and negative cases is quantified by the area under the ROC curve (AUC). To perform a full ROC curve analysis, the predicted probabilities are first saved and next used as a new variable in ROC curve analysis. The dependent variable used in logistic regression then acts as the classification variable in the ROC curve analysis dialog box.

Box plots encompassing the median (dark horizontal line) of log-normalized gene expression values for particular microRNAs were generated using Statistica software (version 9.0; StatSoft, Inc., USA). The upper and lower limits of the boxes represent the 75th and 25th percentiles, respectively. The upper and lower whiskers indicate the maximum and minimum values that are no more than 1.5 times the span of the interquartile range (range of the values between the 25th and the 75th percentiles). Outliers are marked by circles and extremes by asterisks.

\subsection{Information on MicroRNA-Gene-Biological Pathway Interactions}

MiRWalk database (available: http://www.umm.uni-heidelberg.de/apps/zmf/mirwalk/) and the Predicted Target module were used to provide information on predicted targets of those microRNAs that have been found to be dysregulated in whole peripheral blood of patients with a history of GDM. Only those predicted targets involved in particular human biological pathways (insulin signaling pathway, type 1 diabetes mellitus pathway, and type 2 diabetes mellitus pathway) are reported [183]. 
MiRWalk is a comprehensive database that provides information on microRNAs from human, mouse, and rat on their predicted and/or validated target genes. MiRWalk2.0 not only documents miRNA binding sites within the complete sequence of a gene, but also combines this information with a comparison of binding sites resulting from 12 existing miRNA-target prediction programs (DIANA-microTv4.0, DIANA-microT-CDS, miRanda, mirBridge, miRDB4.0, miRmap, miRNAMap, DoRiNA, PicTar2, PITA, RNA22v2, RNAhybrid2.1, and Targetscan6.2) to build novel comparative platforms of binding sites for the promoter (4 prediction datasets), cds (5 prediction datasets), $5^{\prime}$ - (5 prediction datasets), and 3'-UTR (13 prediction datasets) regions. Information on miRNA-target interactions on 2035 disease ontologies (DO), 6727 human phenotype ontologies (HPO) and 4980 OMIM disorders is available. It provides possible interactions between microRNAs and genes associated with 597 KEGG, 456 Panther, and 522 Wiki pathways.

\section{Conclusions}

In conclusion, epigenetic changes characteristic for diabetes mellitus and cardiovascular/ cerebrovascular diseases are also present in whole peripheral blood of a proportion of mothers with a history of GDM. Likewise, a previous occurrence of either GH, PE, and/or FGR, as well as a previous occurrence of GDM, is associated with the upregulation of miR-1-3p, miR-17-5p, miR-20a-5p, miR-20b-5p, miR-29a-3p, miR-100-5p, miR-125b-5p, miR-126-3p, miR-130b-3p, miR-133a-3p, miR-143-3p, miR-145-5p, miR-146a-5p, miR-181a-5p, miR-199a-5p, miR-221-3p, and miR-499a-5p.

On the other hand, upregulation of miR-16-5p, miR-21-5p, miR-23a-3p, miR-24-3p, miR-26a-5p, miR-103a-3p, miR-195-5p, miR-342-3p, and miR-574-3p represents a unique feature of aberrant expression profile of women with a prior exposure to GDM.

Any changes in epigenome (upregulation of miR-16-5p, miR-17-5p, miR-29a-3p, and miR-195-5p) that were induced by GDM-complicated pregnancy are long-acting and may predispose mothers affected with GDM to later development of diabetes mellitus and cardiovascular/cerebrovascular diseases. In addition, novel epigenetic changes (upregulation of a series of microRNAs) appeared in a proportion of women that were exposed to GDM throughout the postpartal life.

Overall, previous occurrence of GDM, therefore, predisposes a proportion of affected individuals to later development of diabetes mellitus and cardiovascular/cerebrovascular diseases. Screening of particular microRNAs may stratify a high-risk group of mothers with a history of GDM that might benefit from implementation of early primary prevention strategies.

Consecutive large-scale studies, including the groups of mothers on diet only and on the combination of diet and therapy, are needed to verify the findings resulting from this particular pilot study.

\section{Patents}

National patent granted-Industrial Property Office, Czech Republic (Patent no. 308178).

International patent filed-Industrial Property Office, Czech Republic (PCT/CZ2019/050051).

Author Contributions: Conceptualization, I.H. and L.K.; methodology, I.H., K.K., and L.D.; software, I.H. and K.K.; validation, I.H. and L.K.; formal analysis, I.H. and K.K.; investigation, K.K. and L.D.; resources, L.D.; data curation, I.H., K.K., and L.D.; writing-original draft preparation, I.H. and K.K.; writing-review and editing, I.H. and K.K.; visualization, K.K.; supervision, I.H. and L.K.; project administration, I.H. and L.K.; funding acquisition, I.H. and L.K. All authors have read and agreed to the published version of the manuscript.

Funding: This research was funded by the Agency of Medical Research, Ministry of Health, Prague, Czech Republic, grant number AZV 16-27761A and by the Charles University, Prague, Czech Republic, grant number 260529/ SVV/2020 and PROGRES Q34. All rights reserved.

Acknowledgments: All procedures were in accordance with the ethical standards of the responsible committee on human experimentation (institutional and national) and with the Helsinki Declaration of 1975, as revised in 2000. We thank the staff of the Institute for the Care of Mother and Child (Katerina Hamplova, Katerina Mackova, Radek Cabela, and Adam Krasny) for assistance with collection of patients' anamnesis. We also thank Jana Kumprichtova, Sarka Stranska, Veronika Marvanova, and Andrea Semencova for assistance with biological sample collection and processing. 
Conflicts of Interest: The authors declare no conflict of interest.

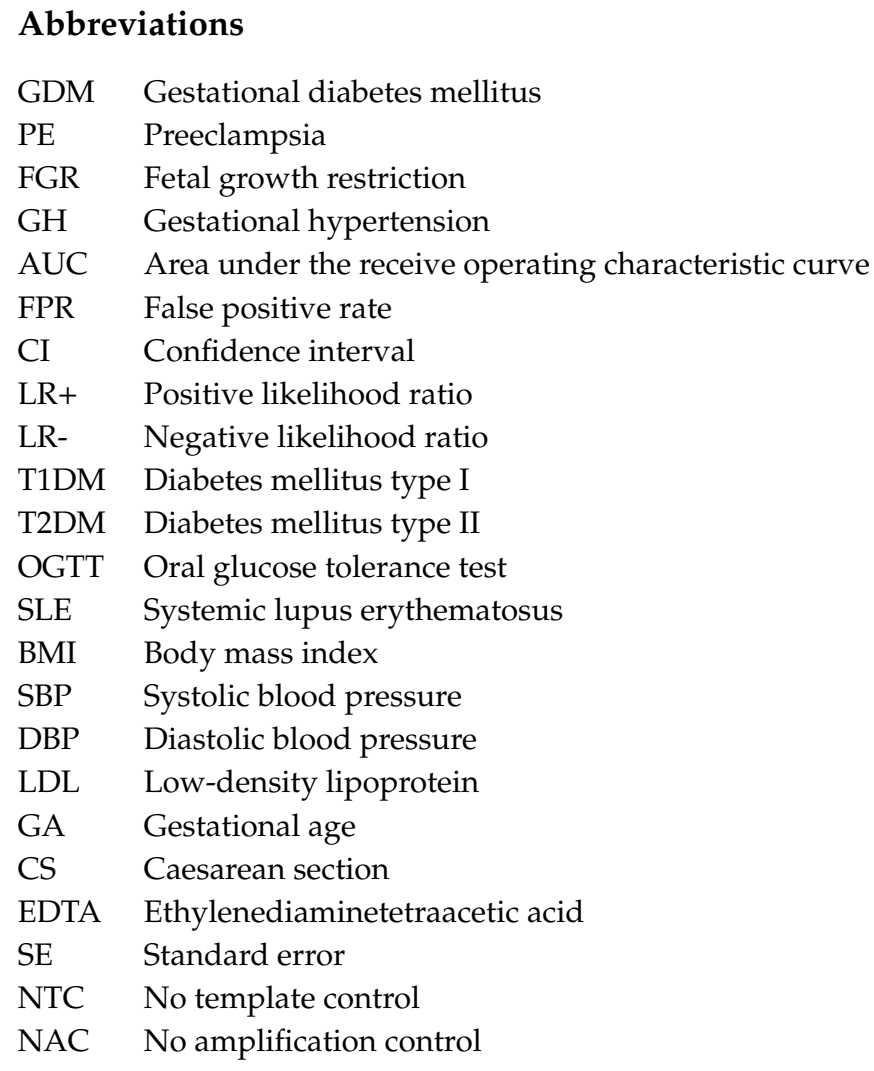

\section{References}

1. Hromadnikova, I.; Kotlabova, K.; Dvorakova, L.; Krofta, L. Postpartum profiling of microRNAs involved in pathogenesis of cardiovascular/cerebrovascular diseases in women exposed to pregnancy-related complications. Int. J. Cardiol. 2019, 291, 158-167. [CrossRef] [PubMed]

2. Hromadnikova, I. Postpartální Epigenetický Profil Kardiovaskulárních mikrorna u Matek Po komplikované Graviditě—Nové Biomarkery Kardiovaskulárního Rizika. Industrial Property Office, Czech Republic. CZ Patent NO. 308178, 31 October 2018.

3. Hromadnikova, I. Postpartum epigenetic profile of cardiovascular microRNAs in women exposed to pregnancy-related complications. Industrial Property Office, Czech Republic. PCT International Application PCT/CZ2019/050051, 30 October 2019.

4. Committee on Practice Bulletins-Obstetrics. ACOG Practice Bulletin No. 190: Gestational Diabetes Mellitus. Obstet. Gynecol. 2018, 131, e49-e64. [CrossRef]

5. England, L.J.; Dietz, P.M.; Njoroge, T.; Callaghan, W.M.; Bruce, C.; Buus, R.M.; Williamson, D.F. Preventing type 2 diabetes: Public health implications for women with a history of gestational diabetes mellitus. Am. J. Obstet. Gynecol. 2009, 200, 365.e1-365.e8. [CrossRef] [PubMed]

6. O'Sullivan, J.B. Body weight and subsequent diabetes mellitus. JAMA 1982, 248, 949-952. [CrossRef] [PubMed]

7. Kim, C.; Newton, K.M.; Knopp, R.H. Gestational diabetes and the incidence of type 2 diabetes: A systematic review. Diabetes Care 2002, 25, 1862-1868. [CrossRef] [PubMed]

8. O'Sullivan, J.B.; Mahan, C.M. Criteria for the oral glucose tolerance test in pregnancy. Diabetes 1964, 13, 278-285. [PubMed]

9. Metzger, B.E.; Buchanan, T.A.; Coustan, D.R.; de Leiva, A.; Dunger, D.B.; Hadden, D.R.; Hod, M.; Kitzmiller, J.L.; Kjos, S.L.; Oats, J.N.; et al. Summary and recommendations of the Fifth International Workshop-Conference on Gestational Diabetes Mellitus. Diabetes Care 2007, 30, S251-S260. [CrossRef] 
10. International Association of Diabetes and Pregnancy Study Groups Consensus Panel; Metzger, B.E.; Gabbe, S.G.; Persson, B.; Buchanan, T.A.; Catalano, P.A.; Damm, P.; Dyer, A.R.; Leiva, A.D.; Hod, M.; et al. International association of diabetes and pregnancy study groups recommendations on the diagnosis and classification of hyperglycemia in pregnancy. Diabetes Care 2010, 33, 676-682. [CrossRef]

11. Li, J.; Dong, X.; Wang, Z.; Wu, J. MicroRNA-1 in Cardiac Diseases and Cancers. Korean J. Physiol. Pharmacol. 2014, 18, 359-363. [CrossRef]

12. Li, Y.Q.; Zhang, M.F.; Wen, H.Y.; Hu, C.L.; Liu, R.; Wei, H.Y.; Ai, C.M.; Wang, G.; Liao, X.X.; Li, X. Comparing the diagnostic values of circulating microRNAs and cardiac troponin $\mathrm{T}$ in patients with acute myocardial infarction. Clinics 2013, 68, 75-80. [CrossRef]

13. Gerlinger-Romero, F.; Yonamine, C.Y.; Junior, D.C.; Esteves, J.V.; Machado, U.F. Dysregulation between TRIM63/FBXO32 expression and soleus muscle wasting in diabetic rats: Potential role of miR-1-3p, -29a/b-3p, and -133a/b-3p. Mol. Cell. Biochem. 2017, 427, 187-199. [CrossRef]

14. Kokkinopoulou, I.; Maratou, E.; Mitrou, P.; Boutati, E.; Sideris, D.C.; Fragoulis, E.G.; Christodoulou, M.I. Decreased expression of microRNAs targeting type-2 diabetes susceptibility genes in peripheral blood of patients and predisposed individuals. Endocrine 2019, 66, 226-239. [CrossRef] [PubMed]

15. Hromadnikova, I.; Kotlabova, K.; Dvorakova, L.; Krofta, L. Evaluation of Vascular Endothelial Function in Young and Middle-Aged Women with Respect to a History of Pregnancy, Pregnancy-Related Complications, Classical Cardiovascular Risk Factors, and Epigenetics. Int. J. Mol. Sci. 2020, 21, E430. [CrossRef] [PubMed]

16. Wang, X.; Shang, Y.; Dai, S.; Wu, W.; Yi, F.; Cheng, L. MicroRNA-16-5p aggravates myocardial infarction injury by targeting expression of insulin receptor substrates 1 and mediating myocardial apoptosis and angiogenesis. Curr. Neurovasc. Res. 2019. [CrossRef] [PubMed]

17. O’Sullivan, J.F.; Neylon, A.; McGorrian, C.; Blake, G.J. miRNA-93-5p and other miRNAs as predictors of coronary artery disease and STEMI. Int. J. Cardiol. 2016, 224, 310-316. [CrossRef] [PubMed]

18. Vegter, E.L.; Schmitter, D.; Hagemeijer, Y.; Ovchinnikova, E.S.; van der Harst, P.; Teerlink, J.R.; O'Connor, C.M.; Metra, M.; Davison, B.A.; Bloomfield, D.; et al. Use of biomarkers to establish potential role and function of circulating microRNAs in acute heart failure. Int. J. Cardiol. 2016, 224, 231-239. [CrossRef] [PubMed]

19. Gacoń, J.; Badacz, R.; Stępień, E.; Karch, I.; Enguita, F.J.; Żmudka, K.; Przewłocki, T.; Kabłak-Ziembicka, A. Diagnostic and prognostic micro-RNAs in ischaemic stroke due to carotid artery stenosis and in acute coronary syndrome: A four-year prospective study. Kardiol. Pol. 2018, 76, 362-369. [CrossRef] [PubMed]

20. Zhu, Y.; Tian, F.; Li, H.; Zhou, Y.; Lu, J.; Ge, Q. Profiling maternal plasma microRNA expression in early pregnancy to predict gestational diabetes mellitus. Int. J. Gynaecol. Obstet. 2015, 130, 49-53. [CrossRef] [PubMed]

21. Cao, Y.L.; Jia, Y.J.; Xing, B.H.; Shi, D.D.; Dong, X.J. Plasma microRNA-16-5p, -17-5p and -20a-5p: Novel diagnostic biomarkers for gestational diabetes mellitus. J. Obstet. Gynaecol. Res. 2017, 43, 974-981. [CrossRef]

22. Duan, Y.R.; Chen, B.P.; Chen, F.; Yang, S.X.; Zhu, C.Y.; Ma, Y.L.; Li, Y.; Shi, J. Exosomal microRNA-16-5p from human urine-derived stem cells ameliorates diabetic nephropathy through protection of podocyte. J. Cell. Mol. Med. 2019. [CrossRef]

23. Assmann, T.S.; Recamonde-Mendoza, M.; Costa, A.R.; Puñales, M.; Tschiedel, B.; Canani, L.H.; Bauer, A.C.; Crispim, D. Circulating miRNAs in diabetic kidney disease: Case-control study and in silico analyses. Acta Diabetol. 2019, 56, 55-65. [CrossRef] [PubMed]

24. Alicka, M.; Major, P.; Wysocki, M.; Marycz, K. Adipose-Derived Mesenchymal Stem Cells Isolated from Patients with Type 2 Diabetes Show Reduced "Stemness" through an Altered Secretome Profile, Impaired Anti-Oxidative Protection, and Mitochondrial Dynamics Deterioration. J. Clin. Med. 2019, 8, E765. [CrossRef] [PubMed]

25. Mogilyansky, E.; Rigoutsos, I. The miR-17/92 cluster: A comprehensive update on its genomics, genetics, functions and increasingly important and numerous roles in health and disease. Cell Death Differ. 2013, 20, 1603-1614. [CrossRef] [PubMed]

26. Zhou, L.; Qi, R.Q.; Liu, M.; Xu, Y.P.; Li, G.; Weiland, M.; Kaplan, D.H.; Mi, Q.S. microRNA miR-17-92 cluster is highly expressed in epidermal Langerhans cells but not required for its development. Genes Immun. 2014, 15, 57-61. [CrossRef] [PubMed]

27. Danielson, L.S.; Park, D.S.; Rotllan, N.; Chamorro-Jorganes, A.; Guijarro, M.V.; Fernandez-Hernando, C.; Fishman, G.I.; Phoon, C.K.; Hernando, E. Cardiovascular dysregulation of miR-17-92 causes a lethal hypertrophic cardiomyopathy and arrhythmogenesis. FASEB J. 2013, 27, 1460-1467. [CrossRef] [PubMed] 
28. Du, W.; Pan, Z.; Chen, X.; Wang, L.; Zhang, Y.; Li, S.; Liang, H.; Xu, C.; Zhang, Y.; Wu, Y.; et al. By targeting Stat3 microRNA-17-5p promotes cardiomyocyte apoptosis in response to ischemia followed by reperfusion. Cell. Physiol. Biochem. 2014, 34, 955-965. [CrossRef]

29. Kaucsár, T.; Révész, C.; Godó, M.; Krenács, T.; Albert, M.; Szalay, C.I.; Rosivall, L.; Benyó, Z.; Bátkai, S.; Thum, T.; et al. Activation of the miR-17 family and miR-21 during murine kidney ischemia-reperfusion injury. Nucleic Acid Ther. 2013, 23, 344-354. [CrossRef]

30. Fang, L.; Ellims, A.H.; Moore, X.L.; White, D.A.; Taylor, A.J.; Chin-Dusting, J.; Dart, A.M. Circulating microRNAs as biomarkers for diffuse myocardial fibrosis in patients with hypertrophic cardiomyopathy. $J$. Transl. Med. 2015, 13, 314. [CrossRef]

31. Wu, J.; Du, K.; Lu, X. Elevated expressions of serum miR-15a, miR-16, and miR-17-5p are associated with acute ischemic stroke. Int. J. Clin. Exp. Med. 2015, 8, 21071-21079.

32. Chen, J.; Xu, L.; Hu, Q.; Yang, S.; Zhang, B.; Jiang, H. MiR-17-5p as circulating biomarkers for the severity of coronary atherosclerosis in coronary artery disease. Int. J. Cardiol. 2015, 197, 123-124. [CrossRef]

33. Tian, L.; Song, Z.; Shao, W.; Du, W.W.; Zhao, L.R.; Zeng, K.; Yang, B.B.; Jin, T. Curcumin represses mouse 3T3-L1 cell adipogenic differentiation via inhibiting miR-17-5p and stimulating the Wnt signalling pathway effector Tcf712. Cell Death Dis. 2017, 8, e2559. [CrossRef] [PubMed]

34. Chen, T.C.; Sung, M.L.; Kuo, H.C.; Chien, S.J.; Yen, C.K.; Chen, C.N. Differential regulation of human aortic smooth muscle cell proliferation by monocyte-derived macrophages from diabetic patients. PLoS ONE 2014, 9, e113752. [CrossRef] [PubMed]

35. Mendell, J.T. miRiad roles for the miR-17-92 cluster in development and disease. Cell 2008, 133, $217-222$. [CrossRef] [PubMed]

36. Brock, M.; Samillan, V.J.; Trenkmann, M.; Schwarzwald, C.; Ulrich, S.; Gay, R.E.; Gassmann, M.; Ostergaard, L.; Gay, S.; Speich, R.; et al. AntagomiR directed against miR-20a restores functional BMPR2 signalling and prevents vascular remodelling in hypoxia-induced pulmonary hypertension. Eur. Heart J. 2014, 35, 3203-3211. [CrossRef] [PubMed]

37. Pheiffer, C.; Dias, S.; Rheeder, P.; Adam, S. Decreased Expression of Circulating miR-20a-5p in South African Women with Gestational Diabetes Mellitus. Mol. Diagn. Ther. 2018, 22, 345-352. [CrossRef] [PubMed]

38. Platania, C.B.M.; Maisto, R.; Trotta, M.C.; D'Amico, M.; Rossi, S.; Gesualdo, C.; D’Amico, G.; Balta, C.; Herman, H.; Hermenean, A.; et al. Retinal and circulating miRNA expression patterns in diabetic retinopathy: An in silico and in vivo approach. Br. J. Pharmacol. 2019, 176, 2179-2194.

39. Lareyre, F.; Clément, M.; Moratal, C.; Loyer, X.; Jean-Baptiste, E.; Hassen-Khodja, R.; Chinetti, G.; Mallat, Z.; Raffort, J. Differential micro-RNA expression in diabetic patients with abdominal aortic aneurysm. Biochimie 2019, 162, 1-7. [CrossRef]

40. Dickinson, B.A.; Semus, H.M.; Montgomery, R.L.; Stack, C.; Latimer, P.A.; Lewton, S.M.; Lynch, J.M.; Hullinger, T.G.; Seto, A.G.; van Rooij, E. Plasma microRNAs serve as biomarkers of therapeutic efficacy and disease progression in hypertension-induced heart failure. Eur. J. Heart Fail. 2013, 15, 650-659. [CrossRef]

41. Flowers, E.; Aouizerat, B.E.; Abbasi, F.; Lamendola, C.; Grove, K.M.; Fukuoka, Y.; Reaven, G.M. Circulating microRNA-320a and microRNA-486 predict thiazolidinedione response: Moving towards precision health for diabetes prevention. Metabolism 2015, 64, 1051-1059. [CrossRef]

42. Katayama, M.; Wiklander, O.P.B.; Fritz, T.; Caidahl, K.; El-Andaloussi, S.; Zierath, J.R.; Krook, A. Circulating Exosomal miR-20b-5p Is Elevated in Type 2 Diabetes and Could Impair Insulin Action in Human Skeletal Muscle. Diabetes 2019, 68, 515-526. [CrossRef]

43. Xiong, Y.; Chen, L.; Yan, C.; Zhou, W.; Endo, Y.; Liu, J.; Hu, L.; Hu, Y.; Mi, B.; Liu, G. Circulating Exosomal miR-20b-5p Inhibition Restores Wnt9b Signaling and Reverses Diabetes-Associated Impaired Wound Healing. Small 2020, 16, e1904044. [CrossRef] [PubMed]

44. Zhu, K.; Hu, X.; Chen, H.; Li, F.; Yin, N.; Liu, A.L.; Shan, K.; Qin, Y.W.; Huang, X.; Chang, Q.; et al. Downregulation of circRNA DMNT3B contributes to diabetic retinal vascular dysfunction through targeting miR-20b-5p and BAMBI. EBioMedicine 2019, 49, 341-353. [CrossRef]

45. Sekar, D.; Venugopal, B.; Sekar, P.; Ramalingam, K. Role of microRNA 21 in diabetes and associated/related diseases. Gene 2016, 582, 14-18. [CrossRef] [PubMed]

46. Suárez, Y.; Fernández-Hernando, C.; Pober, J.S.; Sessa, W.C. Dicer dependent microRNAs regulate gene expression and functions in human endothelial cells. Circ. Res. 2007, 100, 1164-1173. [CrossRef] [PubMed] 
47. Dong, S.; Ma, W.; Hao, B.; Hu, F.; Yan, L.; Yan, X.; Wang, Y.; Chen, Z.; Wang, Z. microRNA-21 promotes cardiac fibrosis and development of heart failure with preserved left ventricular ejection fraction by up-regulating Bcl-2. Int. J. Clin. Exp. Pathol. 2014, 7, 565-574.

48. Zhang, J.; Xing, Q.; Zhou, X.; Li, J.; Li, Y.; Zhang, L.; Zhou, Q.; Tang, B. Circulating miRNA-21 is a promising biomarker for heart failure. Mol. Med. Rep. 2017, 16, 7766-7774. [CrossRef]

49. Licholai, S.; Blaż, M.; Kapelak, B.; Sanak, M. Unbiased Profile of MicroRNA Expression in Ascending Aortic Aneurysm Tissue Appoints Molecular Pathways Contributing to the Pathology. Ann. Thorac. Surg. 2016, 102, 1245-1252. [CrossRef]

50. Kriegel, A.J.; Baker, M.A.; Liu, Y.; Liu, P.; Cowley, A.W., Jr.; Liang, M. Endogenous microRNAs in human microvascular endothelial cells regulate mRNAs encoded by hypertension-related genes. Hypertension 2015, 66, 793-799. [CrossRef]

51. Velle-Forbord, T.; Eidlaug, M.; Debik, J.; Sæther, J.C.; Follestad, T.; Nauman, J.; Gigante, B.; Røsjø, H.; Omland, T.; Langaas, M.; et al. Circulating microRNAs as predictive biomarkers of myocardial infarction: Evidence from the HUNT study. Atherosclerosis 2019, 289, 1-7. [CrossRef]

52. Demirsoy, İ.H.; Ertural, D.Y.; Balci, Ş.; Çınkır, Ü.; Sezer, K.; Tamer, L.; Aras, N. Profiles of Circulating MiRNAs Following Metformin Treatment in Patients with Type 2 Diabetes. J. Med. Biochem. 2018, 37, 499-506. [CrossRef]

53. Olivieri, F.; Spazzafumo, L.; Bonafè, M.; Recchioni, R.; Prattichizzo, F.; Marcheselli, F.; Micolucci, L.; Mensà, E.; Giuliani, A.; Santini, G.; et al. MiR-21-5p and miR-126a-3p levels in plasma and circulating angiogenic cells: Relationship with type 2 diabetes complications. Oncotarget 2015, 6, 35372-35382. [CrossRef] [PubMed]

54. Assmann, T.S.; Recamonde-Mendoza, M.; De Souza, B.M.; Crispim, D. MicroRNA expression profiles and type 1 diabetes mellitus: Systematic review and bioinformatic analysis. Endocr. Connect. 2017, 6, 773-790. [CrossRef] [PubMed]

55. Lakhter, A.J.; Pratt, R.E.; Moore, R.E.; Doucette, K.K.; Maier, B.F.; DiMeglio, L.A.; Sims, E.K. Beta cell extracellular vesicle miR-21-5p cargo is increased in response to inflammatory cytokines and serves as a biomarker of type 1 diabetes. Diabetologia 2018, 61, 1124-1134. [CrossRef] [PubMed]

56. Grieco, G.E.; Cataldo, D.; Ceccarelli, E.; Nigi, L.; Catalano, G.; Brusco, N.; Mancarella, F.; Ventriglia, G.; Fondelli, C.; Guarino, E.; et al. Serum Levels of miR-148a and miR-21-5p Are Increased in Type 1 Diabetic Patients and Correlated with Markers of Bone Strength and Metabolism. Noncoding RNA 2018, 4, E37. [CrossRef]

57. Gholaminejad, A.; Abdul Tehrani, H.; Gholami Fesharaki, M. Identification of candidate microRNA biomarkers in diabetic nephropathy: A meta-analysis of profiling studies. J. Nephrol. 2018, 31, 813-831. [CrossRef]

58. Long, B.; Gan, T.Y.; Zhang, R.C.; Zhang, Y.H. miR-23a Regulates Cardiomyocyte Apoptosis by Targeting Manganese Superoxide Dismutase. Mol. Cells 2017, 40, 542-549. [CrossRef]

59. Wang, S.; He, W.; Wang, C. MiR-23a Regulates the Vasculogenesis of Coronary Artery Disease by Targeting Epidermal Growth Factor Receptor. Cardiovasc. Ther. 2016, 34, 199-208. [CrossRef]

60. Cong, X.; Li, Y.; Lu, N.; Dai, Y.; Zhang, H.; Zhao, X.; Liu, Y. Resveratrol attenuates the inflammatory reaction induced by ischemia/reperfusion in the rat heart. Mol. Med. Rep. 2014, 9, 2528-2532. [CrossRef]

61. Černá, V.; Ostašov, P.; Pitule, P.; Moláček, J.; Třeška, V.; Pešta, M. The Expression Profile of MicroRNAs in Small and Large Abdominal Aortic Aneurysms. Cardiol. Res. Pract. 2019, 2019, 8645840. [CrossRef]

62. Lozano-Bartolomé, J.; Llauradó, G.; Portero-Otin, M.; Altuna-Coy, A.; Rojo-Martínez, G.; Vendrell, J.; Jorba, R.; Rodríguez-Gallego, E.; Chacón, M.R. Altered Expression of miR-181a-5p and miR-23a-3p Is Associated With Obesity and TNF $\alpha$-Induced Insulin Resistance. J. Clin. Endocrinol. Metab. 2018, 103, 1447-1458. [CrossRef]

63. Dolz, S.; Górriz, D.; Tembl, J.I.; Sánchez, D.; Fortea, G.; Parkhutik, V.; Lago, A. Circulating MicroRNAs as Novel Biomarkers of Stenosis Progression in Asymptomatic Carotid Stenosis. Stroke 2017, 48, 10-16. [CrossRef] [PubMed]

64. De Gonzalo-Calvo, D.; Cenarro, A.; Garlaschelli, K.; Pellegatta, F.; Vilades, D.; Nasarre, L.; Camino-Lopez, S.; Crespo, J.; Carreras, F.; Leta, R.; et al. Translating the microRNA signature of microvesicles derived from human coronary artery smooth muscle cells in patients with familial hypercholesterolemia and coronary artery disease. J. Mol. Cell. Cardiol. 2017, 106, 55-67. [CrossRef] [PubMed]

65. Gecys, D.; Tatarunas, V.; Veikutiene, A.; Lesauskaite, V. New potential modulators of CYP4F2 enzyme activity in angina pectoris: Hsa-miR-24-3p and hsa-miR-34a-5p. Biomarkers 2020, 25, 40-47. [CrossRef] [PubMed] 
66. Onrat, S.T.; Onrat, E.; Ercan Onay, E.; Yalım, Z.; Avşar, A. The Genetic Determination of the Differentiation between Ischemic Dilated Cardiomyopathy and Idiopathic Dilated Cardiomyopathy. Genet. Test. Mol. Biomarkers 2018, 22, 644-651. [CrossRef]

67. Tan, H.; Qi, J.; Fan, B.Y.; Zhang, J.; Su, F.F.; Wang, H.T. MicroRNA-24-3p Attenuates Myocardial Ischemia/Reperfusion Injury by Suppressing RIPK1 Expression in Mice. Cell. Physiol. Biochem. 2018, 51, 46-62. [CrossRef]

68. Xiao, X.; Lu, Z.; Lin, V.; May, A.; Shaw, D.H.; Wang, Z.; Che, B.; Tran, K.; Du, H.; Shaw, P.X. MicroRNA miR-24-3p Reduces Apoptosis and Regulates Keap1-Nrf2 Pathway in Mouse Cardiomyocytes Responding to Ischemia/Reperfusion Injury. Oxid. Med. Cell. Longev. 2018, 2018, 7042105. [CrossRef]

69. Gao, J.; Liu, Q.G. The role of miR-26 in tumors and normal tissues. Oncol. Lett. 2011, 2, 1019-1023. [CrossRef]

70. Zheng, L.; Lin, S.; Lv, C. MiR-26a-5p regulates cardiac fibroblasts collagen expression by targeting ULK1. Sci. Rep. 2018, 8, 2104. [CrossRef]

71. Bye, A.; Røsjø, H.; Nauman, J.; Silva, G.J.; Follestad, T.; Omland, T.; Wisløff, U. Circulating microRNAs predict future fatal myocardial infarction in healthy individuals - The HUNT study. J. Mol. Cell. Cardiol. 2016, 97, 162-168. [CrossRef]

72. Hsu, A.; Chen, S.J.; Chang, Y.S.; Chen, H.C.; Chu, P.H. Systemic approach to identify serum microRNAs as potential biomarkers for acute myocardial infarction. Biomed Res. Int. 2014. [CrossRef]

73. Xing, X.; Guo, S.; Zhang, G.; Liu, Y.; Bi, S.; Wang, X.; Lu, Q. miR-26a-5p protects against myocardial ischemia/reperfusion injury by regulating the PTEN/PI3K/AKT signaling pathway. Braz. J. Med. Biol. Res. 2020, 53, e9106. [CrossRef] [PubMed]

74. Chouvarine, P.; Geldner, J.; Giagnorio, R.; Legchenko, E.; Bertram, H.; Hansmann, G. Trans-Right-Ventricle and Transpulmonary MicroRNA Gradients in Human Pulmonary Arterial Hypertension. Pediatr. Crit. Care Med. 2019. [CrossRef] [PubMed]

75. Garavelli, S.; Bruzzaniti, S.; Tagliabue, E.; Prattichizzo, F.; Di Silvestre, D.; Perna, F.; La Sala, L.; Ceriello, A.; Mozzillo, E.; Fattorusso, V.; et al. Blood Co-Circulating Extracellular microRNAs and Immune Cell Subsets Associate with Type 1 Diabetes Severity. Int. J. Mol. Sci. 2020, 21, E477. [CrossRef] [PubMed]

76. Ye, Y.; Hu, Z.; Lin, Y.; Zhang, C.; Perez-Polo, J.R. Downregulation of microRNA-29 by antisense inhibitors and a PPAR-gamma agonist protects against myocardial ischaemia-reperfusion injury. Cardiovasc. Res. 2010, 87, 535-544. [CrossRef] [PubMed]

77. Moraes, L.N.; Fernandez, G.J.; Vechetti-Júnior, I.J.; Freire, P.P.; Souza, R.W.A.; Villacis, R.A.R.; Rogatto, S.R.; Reis, P.P.; Dal-Pai-Silva, M.; Carvalho, R.F. Integration of miRNA and mRNA expression profiles reveals microRNA-regulated networks during muscle wasting in cardiac cachexia. Sci. Rep. 2017, 7, 6998. [CrossRef]

78. Zhao, Y.; Yuan, Y.; Qiu, C. Underexpression of CACNA1C Caused by Overexpression of microRNA-29a Underlies the Pathogenesis of Atrial Fibrillation. Med. Sci. Monit. 2016, 22, 2175-2181. [CrossRef]

79. Zhang, L.; Zhang, Y.; Xue, S.; Ding, H.; Wang, Y.; Qi, H.; Wang, Y.; Zhu, W.; Li, P. Clinical significance of circulating microRNAs as diagnostic biomarkers for coronary artery disease. J. Cell. Mol. Med. 2020, 24, 1146-1150. [CrossRef]

80. Wander, P.L.; Boyko, E.J.; Hevner, K.; Parikh, V.J.; Tadesse, M.G.; Sorensen, T.K.; Williams, M.A.; Enquobahrie, D.A. Circulating early- and mid-pregnancy microRNAs and risk of gestational diabetes. Diabetes Res. Clin. Pract. 2017, 132, 1-9. [CrossRef]

81. Kong, L.; Zhu, J.; Han, W.; Jiang, X.; Xu, M.; Zhao, Y.; Dong, Q.; Pang, Z.; Guan, Q.; Gao, L.; et al. Significance of serum microRNAs in pre-diabetes and newly diagnosed type 2 diabetes: A clinical study. Acta Diabetol. 2011, 48, 61-69. [CrossRef]

82. Widlansky, M.E.; Jensen, D.M.; Wang, J.; Liu, Y.; Geurts, A.M.; Kriegel, A.J.; Liu, P.; Ying, R.; Zhang, G.; Casati, M.; et al. miR-29 contributes to normal endothelial function and can restore it in cardiometabolic disorders. EMBO Mol. Med. 2018, 10, E8046. [CrossRef]

83. Moncini, S.; Salvi, A.; Zuccotti, P.; Viero, G.; Quattrone, A.; Barlati, S.; De Petro, G.; Venturin, M.; Riva, P. The role of miR-103 and miR-107 in regulation of CDK5R1 expression and in cellular migration. PLoS ONE 2011, 6, e20038. [CrossRef]

84. Huang, L.; Li, L.; Chen, X.; Zhang, H.; Shi, Z. MiR-103a targeting Piezo1 is involved in acute myocardial infarction through regulating endothelium function. Cardiol. J. 2016, 23, 556-562. [CrossRef] [PubMed] 
85. Deng, B.; Du, J.; Hu, R.; Wang, A.P.; Wu, W.H.; Hu, C.P.; Li, Y.J.; Li, X.H. MicroRNA-103/107 is involved in hypoxia-induced proliferation of pulmonary arterial smooth muscle cells by targeting HIF-1ß. Life Sci. 2016, 147, 117-124. [CrossRef] [PubMed]

86. Trajkovski, M.; Hausser, J.; Soutschek, J.; Bhat, B.; Akin, A.; Zavolan, M.; Heim, M.H.; Stoffel, M. MicroRNAs 103 and 107 regulate insulin sensitivity. Nature 2011, 474, 649-653. [CrossRef] [PubMed]

87. Assmann, T.S.; Recamonde-Mendoza, M.; Puñales, M.; Tschiedel, B.; Canani, L.H.; Crispim, D. MicroRNA expression profile in plasma from type 1 diabetic patients: Case-control study and bioinformatic analysis. Diabetes Res. Clin. Pract. 2018, 141, 35-46. [CrossRef]

88. Shaham, L.; Binder, V.; Gefen, N.; Borkhardt, A.; Izraeli, S. MiR-125 in normal and malignant hematopoiesis. Leukemia 2012, 26, 2011-2018. [CrossRef]

89. Tiedt, S.; Prestel, M.; Malik, R.; Schieferdecker, N.; Duering, M.; Kautzky, V.; Stoycheva, I.; Böck, J.; Northoff, B.H.; Klein, M.; et al. RNA-Seq Identifies Circulating miR-125a-5p, miR-125b-5p, and miR-143-3p as Potential Biomarkers for Acute Ischemic Stroke. Circ. Res. 2017, 121, 970-980. [CrossRef]

90. Jia, K.; Shi, P.; Han, X.; Chen, T.; Tang, H.; Wang, J. Diagnostic value of miR-30d-5p and miR-125b-5p in acute myocardial infarction. Mol. Med. Rep. 2016, 14, 184-194. [CrossRef]

91. Bayoumi, A.S.; Park, K.M.; Wang, Y.; Teoh, J.P.; Aonuma, T.; Tang, Y.; Su, H.; Weintraub, N.L.; Kim, I.M. A carvedilol-responsive microRNA, miR-125b-5p protects the heart from acute myocardial infarction by repressing pro-apoptotic bak1 and klf13 in cardiomyocytes. J. Mol. Cell. Cardiol. 2018, 114, 72-82. [CrossRef]

92. Lamadrid-Romero, M.; Solís, K.H.; Cruz-Reséndiz, M.S.; Pérez, J.E.; Díaz, N.F.; Flores-Herrera, H.; García-López, G.; Perichart, O.; Reyes-Muñoz, E.; Arenas-Huertero, F.; et al. Central nervous system development-related microRNAs levels increase in the serum of gestational diabetic women during the first trimester of pregnancy. Neurosci. Res. 2018, 130, 8-22. [CrossRef]

93. Satake, E.; Pezzolesi, M.G.; Md Dom, Z.I.; Smiles, A.M.; Niewczas, M.A.; Krolewski, A.S. Circulating miRNA Profiles Associated With Hyperglycemia in Patients With Type 1 Diabetes. Diabetes 2018, 67, 1013-1023. [CrossRef] [PubMed]

94. Samandari, N.; Mirza, A.H.; Kaur, S.; Hougaard, P.; Nielsen, L.B.; Fredheim, S.; Mortensen, H.B.; Pociot, F. Influence of Disease Duration on Circulating Levels of miRNAs in Children and Adolescents with New Onset Type 1 Diabetes. Noncoding RNA 2018, 4, E35. [CrossRef] [PubMed]

95. Yu, C.Y.; Yang, C.Y.; Rui, Z.L. MicroRNA-125b-5p improves pancreatic $\beta$-cell function through inhibiting JNK signaling pathway by targeting DACT1 in mice with type 2 diabetes mellitus. Life Sci. 2019, 224, 67-75. [CrossRef] [PubMed]

96. Wu, X.J.; Zhao, Z.F.; Kang, X.J.; Wang, H.J.; Zhao, J.; Pu, X.M. MicroRNA-126-3p suppresses cell proliferation by targeting PIK3R2 in Kaposi's sarcoma cells. Oncotarget 2016, 7, 36614-36621. [PubMed]

97. Matsha, T.E.; Kengne, A.P.; Hector, S.; Mbu, D.L.; Yako, Y.Y.; Erasmus, R.T. MicroRNA profiling and their pathways in South African individuals with prediabetes and newly diagnosed type 2 diabetes mellitus. Oncotarget 2018, 9, 30485-30498. [CrossRef]

98. Tryggestad, J.B.; Vishwanath, A.; Jiang, S.; Mallappa, A.; Teague, A.M.; Takahashi, Y.; Thompson, D.M.; Chernausek, S.D. Influence of gestational diabetes mellitus on human umbilical vein endothelial cell miRNA. Clin. Sci. 2016, 130, 1955-1967. [CrossRef]

99. Lan, X.; Wu, L.; Wu, N.; Chen, Q.; Li, Y.; Du, X.; Wei, C.; Feng, L.; Li, Y.; Osoro, E.K.; et al. Long Noncoding RNA lnc-HC Regulates PPAR $\gamma$-Mediated Hepatic Lipid Metabolism through miR-130b-3p. Mol. Ther. Nucleic Acids 2019, 18, 954-965. [CrossRef]

100. Zhang, J.; Jazii, F.R.; Haghighi, M.M.; Alvares, D.; Liu, L.; Khosraviani, N.; Adeli, K. miR-130b is a potent stimulator of hepatic very-low-density lipoprotein assembly and secretion via marked induction of microsomal triglyceride transfer protein. Am. J. Physiol. Endocrinol. Metab. 2020, 318, E262-E275. [CrossRef]

101. Li, P.; Zhang, Q.; Wu, X.; Yang, X.; Zhang, Y.; Li, Y.; Jiang, F. Circulating microRNAs serve as novel biological markers for intracranial aneurysms. J. Am. Heart Assoc. 2014, 3, e000972. [CrossRef]

102. Tian, C.; Li, Z.; Yang, Z.; Huang, Q.; Liu, J.; Hong, B. Plasma MicroRNA-16 Is a Biomarker for Diagnosis, Stratification, and Prognosis of Hyperacute Cerebral Infarction. PLoS ONE 2016, 11, e0166688. [CrossRef]

103. Prabu, P.; Rome, S.; Sathishkumar, C.; Aravind, S.; Mahalingam, B.; Shanthirani, C.S.; Gastebois, C.; Villard, A.; Mohan, V.; Balasubramanyam, M. Circulating MiRNAs of 'Asian Indian Phenotype' Identified in Subjects with Impaired Glucose Tolerance and Patients with Type 2 Diabetes. PLoS ONE 2015, 10, e0128372. [CrossRef] [PubMed] 
104. Feng, T.; Li, K.; Zheng, P.; Wang, Y.; Lv, Y.; Shen, L.; Chen, Y.; Xue, Z.; Li, B.; Jin, L.; et al. Weighted Gene Coexpression Network Analysis Identified MicroRNA Coexpression Modules and Related Pathways in Type 2 Diabetes Mellitus. Oxid. Med. Cell. Longev. 2019. [CrossRef] [PubMed]

105. Liang, H.W.; Yang, X.; Wen, D.Y.; Gao, L.; Zhang, X.Y.; Ye, Z.H.; Luo, J.; Li, Z.Y.; He, Y.; Pang, Y.Y.; et al. Utility of miR-133a-3p as a diagnostic indicator for hepatocellular carcinoma: An investigation combined with GEO, TCGA, meta-analysis and bioinformatics. Mol. Med. Rep. 2018, 17, 1469-1484. [CrossRef] [PubMed]

106. Van Rooij, E.; Olson, E.N. MicroRNAs: Powerful new regulators of heart disease and provocative therapeutic targets. J. Clin. Investig. 2007, 117, 2369-2376. [CrossRef]

107. Wang, J.; Xu, R.; Lin, F.; Zhang, S.; Zhang, G.; Hu, S.; Zheng, Z. MicroRNA: Novel regulators involved in the remodeling and reverse remodeling of the heart. Cardiology 2009, 113, 81-88. [CrossRef]

108. Kukreja, R.C.; Yin, C.; Salloum, F.N. MicroRNAs: New players in cardiac injury and protection. Mol. Pharmacol. 2011, 80, 558-564. [CrossRef]

109. Duisters, R.F.; Tijsen, A.J.; Schroen, B.; Leenders, J.J.; Lentink, V.; van der Made, I.; Herias, V.; van Leeuwen, R.E.; Schellings, M.W.; Barenbrug, P.; et al. miR-133 and miR-30 regulate connective tissue growth factor: Implications for a role of microRNAs in myocardial matrix remodeling. Circ. Res. 2009, 104, 170-178. [CrossRef]

110. Liu, W.; Ling, S.; Sun, W.; Liu, T.; Li, Y.; Zhong, G.; Zhao, D.; Zhang, P.; Song, J.; Jin, X.; et al. Circulating microRNAs correlated with the level of coronary artery calcification in symptomatic patients. Sci. Rep. 2015, 5, 16099. [CrossRef]

111. Jiang, Y.; Zhang, M.; He, H.; Chen, J.; Zeng, H.; Li, J.; Duan, R. MicroRNA/mRNA profiling and regulatory network of intracranial aneurysm. BMC Med. Genomics 2013, 6, 36. [CrossRef]

112. Liu, H.; Xiong, W.; Liu, F.; Lin, F.; He, J.; Liu, C.; Lin, Y.; Dong, S. Significant role and mechanism of microRNA-143-3p/KLLN axis in the development of coronary heart disease. Am. J. Transl. Res. 2019, 11, 3610-3619.

113. Li, C.; Li, J.; Xue, K.; Zhang, J.; Wang, C.; Zhang, Q.; Chen, X.; Gao, C.; Yu, X.; Sun, L. MicroRNA-143-3p promotes human cardiac fibrosis via targeting sprouty3 after myocardial infarction. J. Mol. Cell. Cardiol. 2019, 129, 281-292. [CrossRef] [PubMed]

114. Yu, B.; Zhao, Y.; Zhang, H.; Xie, D.; Nie, W.; Shi, K. Inhibition of microRNA-143-3p attenuates myocardial hypertrophy by inhibiting inflammatory response. Cell Biol. Int. 2018, 42, 1584-1593. [CrossRef] [PubMed]

115. Jiao, M.; You, H.Z.; Yang, X.Y.; Yuan, H.; Li, Y.L.; Liu, W.X.; Jin, M.; Du, J. Circulating microRNA signature for the diagnosis of childhood dilated cardiomyopathy. Sci. Rep. 2018, 8, 724. [CrossRef]

116. Deng, L.; Blanco, F.J.; Stevens, H.; Lu, R.; Caudrillier, A.; McBride, M.; McClure, J.D.; Grant, J.; Thomas, M.; Frid, M.; et al. MicroRNA-143 Activation Regulates Smooth Muscle and Endothelial Cell Crosstalk in Pulmonary Arterial Hypertension. Circ. Res. 2015, 117, 870-883. [CrossRef] [PubMed]

117. Shi, L.; Tian, C.; Sun, L.; Cao, F.; Meng, Z. The lncRNA TUG1/miR-145-5p/FGF10 regulates proliferation and migration in VSMCs of hypertension. Biochem. Biophys. Res. Commun. 2018, 501, 688-695. [CrossRef]

118. Yang, X.; Niu, X.; Xiao, Y.; Lin, K.; Chen, X. MiRNA expression profiles in healthy OSAHS and OSAHS with arterial hypertension: Potential diagnostic and early warning markers. Respir. Res. 2018, 19, 194. [CrossRef]

119. Toro, R.; Blasco-Turrión, S.; Morales-Ponce, F.J.; Gonzalez, P.; Martínez-Camblor, P.; López-Granados, A.; Brugada, R.; Campuzano, O.; Pérez-Serra, A.; Rosa Longobardo, F.; et al. Plasma microRNAs as biomarkers for Lamin A/C-related dilated cardiomyopathy. J. Mol. Med. 2018, 96, 845-856. [CrossRef]

120. Yuan, M.; Zhang, L.; You, F.; Zhou, J.; Ma, Y.; Yang, F.; Tao, L. MiR-145-5p regulates hypoxia-induced inflammatory response and apoptosis in cardiomyocytes by targeting CD40. Mol. Cell. Biochem. 2017, 431, 123-131. [CrossRef]

121. Wu, G.; Tan, J.; Li, J.; Sun, X.; Du, L.; Tao, S. miRNA-145-5p induces apoptosis after ischemia-reperfusion by targeting dual specificity phosphatase 6. J. Cell. Physiol. 2019, 234, 16281-16289. [CrossRef]

122. Xie, X.; Peng, L.; Zhu, J.; Zhou, Y.; Li, L.; Chen, Y.; Yu, S.; Zhao, Y. miR-145-5p/Nurr1/TNF- $\alpha$ Signaling-Induced Microglia Activation Regulates Neuron Injury of Acute Cerebral Ischemic/Reperfusion in Rats. Front. Mol. Neurosci. 2017, 10, 383. [CrossRef]

123. Nunez Lopez, Y.O.; Retnakaran, R.; Zinman, B.; Pratley, R.E.; Seyhan, A.A. Predicting and understanding the response to short-term intensive insulin therapy in people with early type 2 diabetes. Mol. Metab. 2019, 20, 63-78. [CrossRef] [PubMed] 
124. Zhang, J.; Cui, C.; Xu, H. Downregulation of miR-145-5p elevates retinal ganglion cell survival to delay diabetic retinopathy progress by targeting FGF5. Biosci. Biotechnol. Biochem. 2019, 83, 1655-1662. [CrossRef]

125. Zamanian Azodi, M.; Rezaei-Tavirani, M.; Rezaei-Tavirani, M.; Robati, R.M. Gestational Diabetes Mellitus Regulatory Network Identifies hsa-miR-145-5p and hsa-miR-875-5p as Potential Biomarkers. Int. J. Endocrinol. Metab. 2019, 17, e86640. [CrossRef]

126. Taganov, K.D.; Boldin, M.P.; Chang, K.J.; Baltimore, D. NF-kappaB-dependent induction of microRNA miR-146, an inhibitor targeted to signaling proteins of innate immune responses. Proc. Natl. Acad. Sci. USA 2006, 103, 12481-12486. [CrossRef]

127. Paterson, M.R.; Kriegel, A.J. MiR-146a/b: A family with shared seeds and different roots. Physiol. Genomics 2017, 49, 243-252. [CrossRef]

128. Zhang, X.; Ye, Z.H.; Liang, H.W.; Ren, F.H.; Li, P.; Dang, Y.W.; Chen, G. Down-regulation of miR-146a-5p and its potential targets in hepatocellular carcinoma validated by a TCGA- and GEO-based study. FEBS Open Bio. 2017, 7, 504-521. [CrossRef] [PubMed]

129. Wang, X.; Ha, T.; Liu, L.; Zou, J.; Zhang, X.; Kalbfleisch, J.; Gao, X.; Williams, D.; Li, C. Increased expression of microRNA-146a decreases myocardial ischaemia/reperfusion injury. Cardiovasc. Res. 2013, 97, 432-442. [CrossRef] [PubMed]

130. Quan, X.; Ji, Y.; Zhang, C.; Guo, X.; Zhang, Y.; Jia, S.; Ma, W.; Fan, Y.; Wang, C. Circulating MiR-146a May be a Potential Biomarker of Coronary Heart Disease in Patients with Subclinical Hypothyroidism. Cell. Physiol. Biochem. 2018, 45, 226-236. [CrossRef] [PubMed]

131. Li, S.H.; Chen, L.; Pang, X.M.; Su, S.Y.; Zhou, X.; Chen, C.Y.; Huang, L.G.; Li, J.P.; Liu, J.L. Decreased miR-146a expression in acute ischemic stroke directly targets the Fbxl10 mRNA and is involved in modulating apoptosis. Neurochem. Int. 2017, 107, 156-167. [CrossRef]

132. Sun, X.; Sit, A.; Feinberg, M.W. Role of miR-181 family in regulating vascular inflammation and immunity. Trends Cardiovasc. Med. 2014, 24, 105-112. [CrossRef]

133. Hulsmans, M.; Sinnaeve, P.; Van der Schueren, B.; Mathieu, C.; Janssens, S.; Holvoet, P. Decreased miR-181a expression in monocytes of obese patients is associated with the occurrence of metabolic syndrome and coronary artery disease. J. Clin. Endocrinol. Metab. 2012, 97, E1213-E1218. [CrossRef] [PubMed]

134. Du, X.; Yang, Y.; Xu, C.; Peng, Z.; Zhang, M.; Lei, L.; Gao, W.; Dong, Y.; Shi, Z.; Sun, X.; et al. Upregulation of miR-181a impairs hepatic glucose and lipid homeostasis. Oncotarget 2017, 8, 91362-91378. [CrossRef] [PubMed]

135. Wu, J.; Fan, C.L.; Ma, L.J.; Liu, T.; Wang, C.; Song, J.X.; Lv, Q.S.; Pan, H.; Zhang, C.N.; Wang, J.J. Distinctive expression signatures of serum microRNAs in ischaemic stroke and transient ischaemic attack patients. Thromb. Haemost. 2017, 117, 992-1001.

136. Zhu, J.; Yao, K.; Wang, Q.; Guo, J.; Shi, H.; Ma, L.; Liu, H.; Gao, W.; Zou, Y.; Ge, J. Circulating miR-181a as a Potential Novel Biomarker for Diagnosis of Acute Myocardial Infarction. Cell. Physiol. Biochem. 2016, 40, 1591-1602. [CrossRef] [PubMed]

137. Nabih, E.S.; Andrawes, N.G. The Association Between Circulating Levels of miRNA-181a and Pancreatic Beta Cells Dysfunction via SMAD7 in Type 1 Diabetic Children and Adolescents. J. Clin. Lab. Anal. 2016, 30, 727-731. [CrossRef] [PubMed]

138. He, J.F.; Luo, Y.M.; Wan, X.H.; Jiang, D. Biogenesis of MiRNA-195 and its role in biogenesis, the cell cycle, and apoptosis. J. Biochem. Mol. Toxicol. 2011, 25, 404-408. [CrossRef]

139. Van Rooij, E.; Sutherland, L.B.; Liu, N.; Williams, A.H.; McAnally, J.; Gerard, R.D.; Richardson, J.A.; Olson, E.N. A signature pattern of stress-responsive microRNAs that can evoke cardiac hypertrophy and heart failure. Proc. Natl. Acad. Sci. USA 2006, 103, 18255-18260. [CrossRef]

140. You, X.Y.; Huang, J.H.; Liu, B.; Liu, S.J.; Zhong, Y.; Liu, S.M. HMGA1 is a new target of miR-195 involving isoprenaline-induced cardiomyocyte hypertrophy. Biochemistry 2014, 79, 538-544. [CrossRef]

141. Zampetaki, A.; Attia, R.; Mayr, U.; Gomes, R.S.; Phinikaridou, A.; Yin, X.; Langley, S.R.; Willeit, P.; Lu, R.; Fanshawe, B.; et al. Role of miR-195 in aortic aneurysmal disease. Circ. Res. 2014, 115, 857-866. [CrossRef]

142. Du, J.; Zheng, R.; Xiao, F.; Zhang, S.; He, K.; Zhang, J.; Shao, Y. Downregulated MicroRNA-195 in the Bicuspid Aortic Valve Promotes Calcification of Valve Interstitial Cells via Targeting SMAD7. Cell. Physiol. Biochem. 2017, 44, 884-896. [CrossRef] 
143. Tagoma, A.; Alnek, K.; Kirss, A.; Uibo, R.; Haller-Kikkatalo, K. MicroRNA profiling of second trimester maternal plasma shows upregulation of miR-195-5p in patients with gestational diabetes. Gene 2018, 672, 137-142. [CrossRef] [PubMed]

144. Lynch, S.M.; Ward, M.; McNulty, H.; Angel, C.Z.; Horigan, G.; Strain, J.J.; Purvis, J.; Tackett, M.; McKenna, D.J. Serum levels of miR-199a-5p correlates with blood pressure in premature cardiovascular disease patients homozygous for the MTHFR 677C > T polymorphism. Genomics 2020, 112, 669-676. [CrossRef] [PubMed]

145. Zhou, Y.; Pang, B.; Xiao, Y.; Zhou, S.; He, B.; Zhang, F.; Liu, W.; Peng, H.; Li, P. The protective microRNA-199a-5p-mediated unfolded protein response in hypoxic cardiomyocytes is regulated by STAT3 pathway. J. Physiol. Biochem. 2019, 75, 73-81. [CrossRef]

146. Liu, Y.; Liu, G.; Zhang, H.; Wang, J. MiRNA-199a-5p influences pulmonary artery hypertension via downregulating Smad3. Biochem. Biophys. Res. Commun. 2016, 473, 859-866. [CrossRef] [PubMed]

147. Wang, J.; Yu, G. A Systems Biology Approach to Characterize Biomarkers for Blood Stasis Syndrome of Unstable Angina Patients by Integrating MicroRNA and Messenger RNA Expression Profiling. Evid.-Based Complement. Alternat. Med 2013. [CrossRef] [PubMed]

148. Massaro, J.D.; Polli, C.D.; Costa ESilva, M.; Alves, C.C.; Passos, G.A.; Sakamoto-Hojo, E.T.; Rodrigues de Holanda Miranda, W.; Bispo Cezar, N.J.; Rassi, D.M.; Crispim, F.; et al. Post-transcriptional markers associated with clinical complications in Type 1 and Type 2 diabetes mellitus. Mol. Cell. Endocrinol. 2019, 490, 1-14. [CrossRef]

149. Collares, C.V.; Evangelista, A.F.; Xavier, D.J.; Rassi, D.M.; Arns, T.; Foss-Freitas, M.C.; Foss, M.C.; Puthier, D.; Sakamoto-Hojo, E.T.; Passos, G.A.; et al. Identifying common and specific microRNAs expressed in peripheral blood mononuclear cell of type 1, type 2, and gestational diabetes mellitus patients. BMC Res. Notes 2013, 6 , 491. [CrossRef] [PubMed]

150. Derda, A.A.; Pfanne, A.; Bär, C.; Schimmel, K.; Kennel, P.J.; Xiao, K.; Schulze, P.C.; Bauersachs, J.; Thum, T. Blood-based microRNA profiling in patients with cardiac amyloidosis. PLoS ONE 2018, 13, e0204235. [CrossRef]

151. Verjans, R.; Peters, T.; Beaumont, F.J.; van Leeuwen, R.; van Herwaarden, T.; Verhesen, W.; Munts, C.; Bijnen, M.; Henkens, M.; Diez, J.; et al. MicroRNA-221/222 Family Counteracts Myocardial Fibrosis in Pressure Overload-Induced Heart Failure. Hypertension 2018, 71, 280-288. [CrossRef]

152. Zhuang, X.; Li, R.; Maimaitijiang, A.; Liu, R.; Yan, F.; Hu, H.; Gao, X.; Shi, H. miR-221-3p inhibits oxidized low-density lipoprotein induced oxidative stress and apoptosis via targeting a disintegrin and metalloprotease-22. J. Cell Biochem. 2019, 120, 6304-6314. [CrossRef]

153. Pereira-da-Silva, T.; Coutinho Cruz, M.; Carrusca, C.; Cruz Ferreira, R.; Napoleão, P.; Mota Carmo, M. Circulating microRNA profiles in different arterial territories of stable atherosclerotic disease: A systematic review. Am. J. Cardiovasc. Dis. 2018, 8, 1-13. [PubMed]

154. Coffey, S.; Williams, M.J.; Phillips, L.V.; Galvin, I.F.; Bunton, R.W.; Jones, G.T. Integrated microRNA and messenger RNA analysis in aortic stenosis. Sci. Rep. 2016, 6, 36904. [CrossRef] [PubMed]

155. Coskunpinar, E.; Cakmak, H.A.; Kalkan, A.K.; Tiryakioglu, N.O.; Erturk, M.; Ongen, Z. Circulating miR-221-3p as a novel marker for early prediction of acute myocardial infarction. Gene 2016, 591, 90-96. [CrossRef] [PubMed]

156. Sørensen, S.S.; Nygaard, A.B.; Nielsen, M.Y.; Jensen, K.; Christensen, T. miRNA expression profiles in cerebrospinal fluid and blood of patients with acute ischemic stroke. Transl. Stroke Res. 2014, 5, 711-718. [CrossRef] [PubMed]

157. Gusar, V.A.; Timofeeva, A.V.; Zhanin, I.S.; Shram, S.I.; Pinelis, V.G. Estimation of Time-Dependent microRNA Expression Patterns in Brain Tissue, Leukocytes, and Blood Plasma of Rats under Photochemically Induced Focal Cerebral Ischemia. Mol. Biol. 2017, 51, 683-695. [CrossRef]

158. Nie, X.; Chen, Y.; Tan, J.; Dai, Y.; Mao, W.; Qin, G.; Ye, S.; Sun, J.; Yang, Z.; Chen, J. MicroRNA-221-3p promotes pulmonary artery smooth muscle cells proliferation by targeting AXIN2 during pulmonary arterial hypertension. Vascul. Pharmacol. 2019, 116, 24-35. [CrossRef]

159. Villard, A.; Marchand, L.; Thivolet, C.; Rome, S. Diagnostic Value of Cell-free Circulating MicroRNAs for Obesity and Type 2 Diabetes: A Meta-analysis. J. Mol. Biomark. Diagn. 2015, 6, 251. [CrossRef]

160. Wang, L.; Xu, L.; Xu, M.; Liu, G.; Xing, J.; Sun, C.; Ding, H. Obesity-Associated MiR-342-3p Promotes Adipogenesis of Mesenchymal Stem Cells by Suppressing CtBP2 and Releasing C/EBP $\alpha$ from CtBP2 Binding. Cell. Physiol. Biochem. 2015, 35, 2285-2298. [CrossRef] 
161. Hezova, R.; Slaby, O.; Faltejskova, P.; Mikulkova, Z.; Buresova, I.; Raja, K.R.; Hodek, J.; Ovesna, J.; Michalek, J. microRNA-342, microRNA-191 and microRNA-510 are differentially expressed in T regulatory cells of type 1 diabetic patients. Cell. Immunol. 2010, 260, 70-74. [CrossRef]

162. Eissa, S.; Matboli, M.; Bekhet, M.M. Clinical verification of a novel urinary microRNA panal: $133 \mathrm{~b},-342$ and -30 as biomarkers for diabetic nephropathy identified by bioinformatics analysis. Biomed. Pharmacother. 2016, 83, 92-99. [CrossRef]

163. Cheng, S.; Cui, Y.; Fan, L.; Mu, X.; Hua, Y. T2DM inhibition of endothelial miR-342-3p facilitates angiogenic dysfunction via repression of FGF11 signaling. Biochem. Biophys. Res. Commun. 2018, 503, 71-78. [CrossRef] [PubMed]

164. Khalyfa, A.; Kheirandish-Gozal, L.; Bhattacharjee, R.; Khalyfa, A.A.; Gozal, D. Circulating microRNAs as Potential Biomarkers of Endothelial Dysfunction in Obese Children. Chest 2016, 149, 786-800. [CrossRef] [PubMed]

165. Hoekstra, M. MicroRNA-499-5p: A therapeutic target in the context of cardiovascular disease. Ann. Transl. Med. 2016, 4, 539. [CrossRef] [PubMed]

166. Zhao, L.; Wang, B.; Zhang, W.; Sun, L. Effect of miR-499a-5p on damage of cardiomyocyte induced by hypoxia-reoxygenation via downregulating CD38 protein. J. Cell. Biochem. 2020, 121, 996-1004. [CrossRef]

167. Neshati, V.; Mollazadeh, S.; Fazly Bazzaz, B.S.; de Vries, A.A.F.; Mojarrad, M.; Naderi-Meshkin, H.; Neshati, Z.; Mirahmadi, M.; Kerachian, M.A. MicroRNA-499a-5p Promotes Differentiation of Human Bone Marrow-Derived Mesenchymal Stem Cells to Cardiomyocytes. Appl. Biochem. Biotechnol. 2018, 186, 245-255. [CrossRef]

168. Boštjančič, E.; Zidar, N.; Glavač, D. MicroRNAs and cardiac sarcoplasmic reticulum calcium ATPase-2 in human myocardial infarction: Expression and bioinformatic analysis. BMC Genomics 2012, 13, 552. [CrossRef]

169. Salinas, J.; Lin, H.; Aparico, H.J.; Huan, T.; Liu, C.; Rong, J.; Beiser, A.; Himali, J.J.; Freedman, J.E.; Larson, M.G.; et al. Whole blood microRNA expression associated with stroke: Results from the Framingham Heart Study. PLoS ONE 2019, 14, e0219261. [CrossRef]

170. Baldeón Rojas, L.; Weigelt, K.; de Wit, H.; Ozcan, B.; van Oudenaren, A.; Sempértegui, F.; Sijbrands, E.; Grosse, L.; van Zonneveld, A.J.; Drexhage, H.A.; et al. Study on inflammation-related genes and microRNAs, with special emphasis on the vascular repair factor HGF and miR-574-3p, in monocytes and serum of patients with T2D. Diabetol. Metab. Syndr. 2016, 8,6.

171. Lai, E.C. Micro RNAs are complementary to 3' UTR sequence motifs that mediate negative post-transcriptional regulation. Nat. Genet. 2002, 30, 363-364. [CrossRef]

172. Guarino, E.; Delli Poggi, C.; Grieco, G.E.; Cenci, V.; Ceccarelli, E.; Crisci, I.; Sebastiani, G.; Dotta, F. Circulating MicroRNAs as Biomarkers of Gestational Diabetes Mellitus: Updates and Perspectives. Int. J. Endocrinol. 2018. [CrossRef]

173. Ibarra, A.; Vega-Guedes, B.; Brito-Casillas, Y.; Wägner, A.M. Diabetes in Pregnancy and MicroRNAs: Promises and Limitations in Their Clinical Application. Noncoding RNA 2018, 4, E32. [CrossRef] [PubMed]

174. Zhao, C.; Dong, J.; Jiang, T.; Shi, Z.; Yu, B.; Zhu, Y.; Chen, D.; Xu, J.; Huo, R.; Dai, J.; et al. Early second-trimester serum miRNA profiling predicts gestational diabetes mellitus. PLoS ONE 2011, 6, e23925. [CrossRef] [PubMed]

175. Xu, K.; Bian, D.; Hao, L.; Huang, F.; Xu, M.; Qin, J.; Liu, Y. microRNA-503 contribute to pancreatic beta cell dysfunction by targeting the mTOR pathway in gestational diabetes mellitus. EXCLI J. 2017, 16, 1177-1187. [PubMed]

176. Sebastiani, G.; Guarino, E.; Grieco, G.E.; Formichi, C.; Delli Poggi, C.; Ceccarelli, E.; Dotta, F. Circulating microRNA (miRNA) Expression Profiling in Plasma of Patients with Gestational Diabetes Mellitus Reveals Upregulation of miRNA miR-330-3p. Front. Endocrinol. 2017, 8, 345. [CrossRef]

177. Hromadnikova, I.; Kotlabova, K.; Dvorakova, L.; Krofta, L.; Sirc, J. Postnatal Expression Profile of microRNAs Associated with Cardiovascular and Cerebrovascular Diseases in Children at the Age of 3 to 11 Years in Relation to Previous Occurrence of Pregnancy-Related Complications. Int. J. Mol. Sci. 2019, 20, E654. [CrossRef]

178. American Diabetes Association. Diagnosis and classification of diabetes mellitus (Position Statement). Diabetes Care 2009, 32, S62-S67. [CrossRef]

179. Metzger, B.E.; Coustan, D.R. Summary and recommendations of the Fourth International WorkshopConference on Gestational Diabetes Mellitus. The Organizing Committee. Diabetes Care 1998, 21, B161-B167. 
180. Livak, K.J.; Schmittgen, T.D. Analysis of relative gene expression data using real-time quantitative PCR and the 2(-Delta Delta C(T)) Method. Methods 2001, 25, 402-408. [CrossRef]

181. Vandesompele, J.; de Preter, K.; Pattyn, F.; Poppe, B.; Van Roy, N.; de Paepe, A.; Speleman, F. Accurate normalization of real-time quantitative RT-PCR data by geometric averaging of multiple internal control genes. Genome Biol. 2002, 3. [CrossRef]

182. Shapiro, S.S.; Wilk, M.B. An Analysis of Variance Test for Normality (Complete Samples). Biometrika 1965, 3/4, 591-611. [CrossRef]

183. Dweep, H.; Sticht, C.; Pandey, P.; Gretz, N. miRWalk—Database: Prediction of possible miRNA binding sites by "walking" the genes of three genomes. J. Biomed. Inform. 2011, 44, 839-847. [CrossRef] [PubMed]

C 2020 by the authors. Licensee MDPI, Basel, Switzerland. This article is an open access article distributed under the terms and conditions of the Creative Commons Attribution (CC BY) license (http://creativecommons.org/licenses/by/4.0/). 